\title{
Platinum-Catalyzed Asymmetric Alkylation of a Secondary Phosphine: Mechanism and Origin of Enantioselectivity
}

\author{
Corina Scriban, ${ }^{a}$ David S. Glueck, ${ }^{a *}$ James A. Golen, ${ }^{b}$ and Arnold L. Rheingold \\ a6128 Burke Laboratory, Department of Chemistry, Dartmouth College, Hanover, New Hampshire, \\ $03755^{\mathrm{b}}$ Department of Chemistry, University of California, San Diego, 9500 Gilman Drive, La \\ Jolla, California, 92093

\section{Supporting Information}

1. Additional Experimental Details

2. NMR Structure Determination of $\mathrm{Pt}((\mathrm{R}, \mathrm{R})-\mathrm{Me}-\mathrm{Duphos})(\mathrm{Ph})(\mathrm{PMeIs})(\mathbf{4})$

3. Details of the X-ray Structure Determinations for $\operatorname{Pt}((\mathrm{R}, \mathrm{R})-\mathrm{Me}-\mathrm{Duphos})(\mathrm{Ph})(\mathrm{OH})(\mathbf{6})$, $\operatorname{Pt}((\mathrm{R}, \mathrm{R})-\mathrm{Me}-\mathrm{Duphos})(\mathrm{Ph})(\mathrm{Br})(\mathbf{7}),\left[\mathrm{Pt}((\mathrm{R}, \mathrm{R})-\mathrm{Me}-\mathrm{Duphos})(\mathrm{Ph})\left(\mathrm{PMeIs}\left(\mathrm{CH}_{2} \mathrm{Ph}\right)\right)\right]\left[\mathrm{BF}_{4}\right] \quad\left(\mathbf{8}-\mathrm{BF}_{4}\right)$, and $(\mathrm{Pt}((\mathrm{R}, \mathrm{R})-\mathrm{Me}-\mathrm{Duphos})(\mathrm{Ph}))_{2}\left(\mu-\mathrm{CO}_{3}\right)$

$\operatorname{Pt}((\mathbf{R}, \mathbf{R})-M e-D u p h o s)(P h)(O M e) . \quad \mathrm{Na}(61.8 \mathrm{mg}, 2.7 \mathrm{mmol})$ was added to stirring $\mathrm{MeOH}$ (2.3 mL, dried over molecular sieves) under nitrogen. Gas evolved and the reaction was allowed to go to completion. $\mathrm{Pt}((\mathrm{R}, \mathrm{R})-\mathrm{Me}-\mathrm{Duphos})(\mathrm{Ph})(\mathrm{Cl})(165 \mathrm{mg}, 0.27 \mathrm{mmol})$ in toluene $(20 \mathrm{~mL})$ was added dropwise to the $\mathrm{NaOMe}$ stirring solution. After $24 \mathrm{~h}$, the solvent was removed under vacuum. The residue was extracted with toluene $(5 \mathrm{~mL})$, then filtered through Celite. The filtrate was concentrated under vacuum, yielding a white sticky residue. Ether $(0.5 \mathrm{~mL})$ was added to the residue giving a white precipitate, which was washed with ether $(3 \times 0.5 \mathrm{~mL})$, and dried under vacuum. Recrystallization from THF/petroleum ether yielded $105 \mathrm{mg}(64 \%)$ of white powder. The methoxide complex could not be isolated in pure form, since it reacted with trace water to yield the hydroxide $\mathrm{Pt}((\mathrm{R}, \mathrm{R})-\mathrm{Me}-\mathrm{Duphos})(\mathrm{Ph})(\mathrm{OH})(\mathbf{6}) .{ }^{31} \mathrm{P}\left\{{ }^{1} \mathrm{H}\right\} \mathrm{NMR}\left(\mathrm{C}_{6} \mathrm{D}_{6}\right): \delta 68.8\left(\mathrm{~d}, \mathrm{~J}=5, \mathrm{~J}_{\mathrm{Pt}-\mathrm{P}}=\right.$ 1798), $47.9\left(\mathrm{~d}, \mathrm{~J}=5, \mathrm{~J}_{\mathrm{Pt}-\mathrm{P}}=3159\right)$. Selected ${ }^{1} \mathrm{H} \mathrm{NMR}\left(\mathrm{C}_{6} \mathrm{D}_{6}\right): \delta 8.09\left(\mathrm{t}, \mathrm{J}=13, \mathrm{~J}_{\mathrm{Pt}-\mathrm{H}}=42,2 \mathrm{H}, \mathrm{Ar}\right)$, 
7.43-7.34 (m, 2H, Ar), $4.25\left(\mathrm{~d}, \mathrm{~J}=6, \mathrm{~J}_{\mathrm{Pt}-\mathrm{H}}=53,3 \mathrm{H}, \mathrm{OMe}\right)$; other peaks overlapped with those due to the hydroxide complex.

$(\operatorname{Pt}((\mathbf{R}, \mathbf{R})-M e-D u p h o s)(P h))_{2}\left(\boldsymbol{\mu}-\mathbf{C O}_{3}\right)$ The hydroxide complex $\mathbf{6}$ did not appear to be sensitive to oxygen, but it took up carbon dioxide from the air. When exposed to air, in solution, after 1 day, it gave $(\mathrm{Pt}((\mathrm{R}, \mathrm{R})-\mathrm{Me}-\mathrm{Duphos})(\mathrm{Ph}))_{2}\left(\mu-\mathrm{CO}_{3}\right)(\sim 10 \%)$, which crystallized out of solution. After 1 month, the ratio hydroxide:carbonate was about 3:1. The carbonate complex was characterized by X-ray crystallography (see below) and ${ }^{31} \mathrm{P}$ NMR spectroscopy $\left({ }^{31} \mathrm{P}\left\{{ }^{1} \mathrm{H}\right\} \mathrm{NMR}\right.$ $\left.\left(\mathrm{C}_{6} \mathrm{D}_{6}\right): \delta 68.7\left(\mathrm{~J}_{\mathrm{Pt}-\mathrm{P}}=1737\right), 47.3\left(\mathrm{~J}_{\mathrm{Pt}-\mathrm{P}}=3728\right)\right)$, but bulk samples were not isolated in pure form. The parent ion was not observed by mass spectroscopy. ESMS m/z: $578.3(\mathrm{Pt}((\mathrm{R}, \mathrm{R})-\mathrm{Me}-$ Duphos $\left.)(\mathrm{Ph})^{+}\right), 613.3,711.3,1182.4$. Attempts to prepare the carbonate complex independently from $\mathrm{Pt}((\mathrm{R}, \mathrm{R})-\mathrm{Me}-\mathrm{Duphos})(\mathrm{Ph})(\mathrm{Cl})$ and $\mathrm{Ag}_{2} \mathrm{CO}_{3}$ in THF, $\mathrm{CH}_{2} \mathrm{Cl}_{2}$ or acetone were unsuccessful; no reaction was observed.

\section{Addition of Benzyl Bromide to Pt((R,R)-Me-Duphos)(Ph)(PMeIs) (4) (modified} workup and measurement of the ee of the phosphine product). A solution of $4(82.7 \mathrm{mg}, 0.1$ mmol) in toluene $(0.5 \mathrm{~mL})$ was transferred into an NMR tube, which was fitted with a septum. The tube was cooled in a $\mathrm{NaCl} /$ ice bath at $-5^{\circ} \mathrm{C}$. Benzyl bromide $(17 \mathrm{mg}, 12 \mu \mathrm{L}, 0.1 \mathrm{mmol})$ was added via a microliter syringe. The reaction mixture was kept at $-5^{\circ} \mathrm{C}$ for $6 \mathrm{~h}$, and at $-15{ }^{\circ} \mathrm{C}$ for $15 \mathrm{~h}$, when the reaction was complete, according to ${ }^{31} \mathrm{P}$ NMR. $\mathrm{Pt}((\mathrm{R}, \mathrm{R})-\mathrm{Me}-\mathrm{Duphos})(\mathrm{Ph})(\mathrm{Br})$ and $\mathrm{PMeIs}\left(\mathrm{CH}_{2} \mathrm{Ph}\right)(\delta-40.4)$ were the only components of the mixture observed in toluene; no precipitate was observed.

The solvent was removed under vacuum and $0.5 \mathrm{~mL}$ of a 9:1 mixture of petroleum ether:THF was added to the residue. The resulting white precipitate was washed with 5 portions of $0.5 \mathrm{~mL}$ of 9:1 petroleum ether:THF. The washes were collected and the solvent was removed under vacuum. The tertiary phosphine was isolated by filtration on a silica column $(5 \mathrm{~cm}$ height, $0.6 \mathrm{~cm}$ diameter), using a 9:1 petroleum ether:THF mixture as eluent, followed by solvent removal under vacuum, yielding $21 \mathrm{mg}$ of colorless liquid (62\% yield, $79 \%$ ee; the ee was determined as described in the Supporting Information for reference 1). ${ }^{1}$ In an analogous experiment carried out at room 
temperature, the phosphine $\mathrm{PMeIs}\left(\mathrm{CH}_{2} \mathrm{Ph}\right)(70 \%$ ee $)$ was isolated in $45 \%$ yield. The white precipitate was dried under vacuum, yielding $68 \mathrm{mg}$ of white powder that consisted of $\mathrm{Pt}((\mathrm{R}, \mathrm{R})-\mathrm{Me}-$ Duphos $)(\mathrm{Ph})(\mathrm{Br})$ contaminated by $\mathrm{PMeIs}\left(\mathrm{CH}_{2} \mathrm{Ph}\right)$, according to the ${ }^{31} \mathrm{P}$ NMR spectrum. A 9:1 mixture of petroleum ether:THF (1 $\mathrm{mL})$ was added to the residue, and the remaining tertiary phosphine was separated from the Pt compound by filtration on a silica column $(5 \mathrm{~cm}$ height, 0.6 cm diameter), using a 9:1 petroleum ether:THF mixture as eluent, followed by solvent removal under vacuum, yielding $9 \mathrm{mg}$ of colorless liquid (88\% total yield). $\mathrm{Pt}((\mathrm{R}, \mathrm{R})-\mathrm{Me}-\mathrm{Duphos})(\mathrm{Ph})(\mathrm{Br})$ was removed from the silica by elution with 6 portions of $0.5 \mathrm{~mL}$ of THF, followed by solvent removal under vacuum, yielding $55 \mathrm{mg}$ of off-white powder of the Pt-bromide complex, contaminated by $\sim 5 \%$ PMeIs $\left(\mathrm{CH}_{2} \mathrm{Ph}\right)$, according to ${ }^{31} \mathrm{P}$ NMR spectroscopy. 
NMR Structure Determination of $\mathrm{Pt}((\mathbf{R}, \mathbf{R})-\mathrm{Me}-\mathrm{Duphos})(\mathrm{Ph})(\mathrm{PMeIs})(\mathbf{4})$ The ${ }^{1} \mathrm{H}$ and ${ }^{13} \mathrm{C}$ NMR spectra of the major diastereomer of 4 in $\mathrm{THF}-\mathrm{d}_{8}$ at $-60{ }^{\circ} \mathrm{C}$ were assigned by onedimensional and two-dimensional $\left({ }^{1} \mathrm{H}-{ }^{31} \mathrm{P}\right.$ correlation, ${ }^{1} \mathrm{H}-{ }^{13} \mathrm{C}$ correlation, ${ }^{1} \mathrm{H}-{ }^{1} \mathrm{H}$ COSY) experiments, as well as ${ }^{1} \mathrm{H}-{ }^{1} \mathrm{H}$ NOESY experiments. The structure in solution is consistent with that observed in the solid state (Figure S1); the atom labelling scheme for this structure is used for the assignments below. Some of this information is repeated from the text of the manuscript for ease of comparison within a single document.

Figure S1. ORTEP diagram of $\mathrm{Pt}((\mathrm{R}, \mathrm{R})-\mathrm{Me}-\mathrm{Duphos})(\mathrm{Ph})(\mathrm{PMeIs})(\mathbf{4})^{2}$

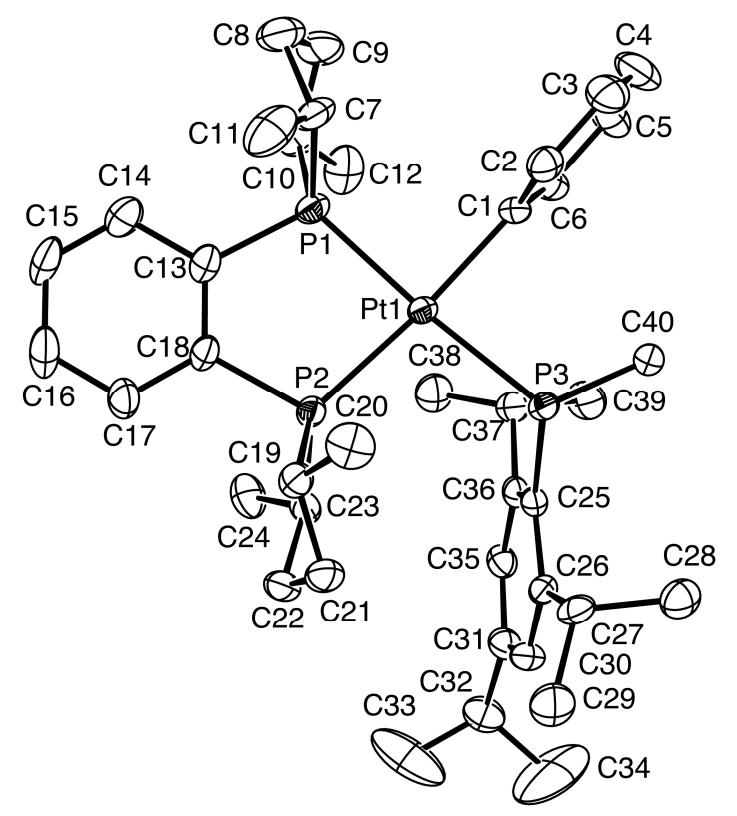

${ }^{1} \mathrm{H}$ NMR $\left(-60{ }^{\circ} \mathrm{C}, \mathrm{THF}-\mathrm{d}_{8}\right.$; the assignments are as in Table S1 and Chart S1, but see the text and the chart footnote for comments on ambiguous assignments): $\delta 7.87-7.86(\mathrm{~m}, 1 \mathrm{H}$, Ar, Duphos, H15), 7.78-7.72 (m, 2H, overlapping Ar, Duphos H16 and Ph ortho H6), 7.56-7.55 (m, 2H, Ar, Duphos, H14/H17), 7.25 (broad t, J = 7, 1H, Ph ortho, H2), 7.07 (broad t, J = 8, 1H, Ph meta, H5), 7.00 (broad t, J = 8, 1H, Ph meta, H3), 6.99 (2H, Is meta, H30/H35), 6.78 (broad t, J = 8, 1H, Ph para, H4), 4.92-4.87 (m, 1H, CH, Is, H27), 4.72-4.70 (m, 1H, CH, Is, H37), 2.94-2.81 (m, 3H, 
overlapping 2CH Duphos (H7/H10) and CH Is (H32)), 2.44-2.39 (m, 1H, CH Duphos, H19), 1.99-1.77 (m, 4H, 2 $\mathrm{CH}_{2}$ ), 1.64-1.53 (m, 4H, $\left.\mathrm{CH}_{2}\right), 1.49$ (dd, J = 17, 7, 3H, $\mathrm{CH}_{3}$ Duphos, H20), 1.39 (dd, $\mathrm{J}=17,10,3 \mathrm{H}, \mathrm{CH}_{3}$ Duphos, H12), 1.36-1.29 (m, 7H, overlapping $2 \mathrm{CH}_{3}$ Is $(\mathrm{H} 29 / \mathrm{H} 38)$ and 1CH Duphos (H23)), 1.23 (broad d, J = 7, 9H, $\left.\mathrm{CH}_{3} \mathrm{Is}, \mathrm{H} 39 / \mathrm{H} 33 / \mathrm{H} 34\right), 1.03$ (d, J = 7, 3H, $\mathrm{CH}_{3}$ Is, H28), 0.77-0.73 (m, 6H, overlapping $\mathrm{CH}_{3}$ Duphos (H11) and P-Me (H40), $\left.\mathrm{J}_{\mathrm{Pt}-\mathrm{H}}=57\right), 0.61$ (dd, $\mathrm{J}=14,7,3 \mathrm{H}, \mathrm{CH}_{3}$ Duphos, H24).

${ }^{13} \mathrm{C}\left\{{ }^{1} \mathrm{H}\right\}$ NMR $\left(-60{ }^{\circ} \mathrm{C}, \mathrm{THF}-\mathrm{d}_{8}\right): \delta 163.0\left(\mathrm{~d}, \mathrm{~J}=95, \mathrm{~J}_{\mathrm{Pt}-\mathrm{C}}=803\right.$, Pt-C (quat Ph, C1)), 155.6 (quat Is, C31), 154.0 (d, J = 26, P-C (quat Is, C25)), 148.1 (2 quat Is, C26/C36), 147.2-146.6 (m, quat Duphos, C18), 144.1-143.3 (m, quat Duphos, C13), 140.2 (d, J = 25, Ph ortho, C6), 137.9 (Ph ortho, C2), 135.2-134.6 (m, Ar, Duphos, C16), 134.5-134.0 (m, Ar, Duphos, C15), 131.9 (d, J = 25, Ar, Duphos, C17), 131.3 (d, J = 25, Ar, Duphos, C14), 128.3 (d, J = 30, Ph meta, C5), 128.1 (d, J = 32, $\mathrm{Ph}$ meta, C3), 122.1 (d, J = 16, Ph para, C4), 121.8 (d, J = 10, Is meta, C30), 120.0 (m, Is meta, C35), 45.1-44.7 (m, CH Duphos, C19), 42.4 (d, J = 32, CH Duphos, C10), 38.1 (broad, $\mathrm{CH}_{2}$ ), 36.9-35.7 (broad, overlapping $\mathrm{CH}_{2}$ ), 35.5 (d, J = 32, CH, Is, C32), 35.1 (d, J = 23, CH, Is, C37), 33.4 (d, J = 34, CH, Is, C27), 32.9 (d, J = 33, CH, Duphos, C7), 32.6 (d, J = 23, CH, Duphos, C23), $28.3\left(\mathrm{CH}_{3}\right.$, Is, C28), $28.1\left(\mathrm{CH}_{3}\right.$, Is, C39), $27.3\left(\mathrm{CH}_{3}\right.$, Is, C38), $25.1\left(\mathrm{CH}_{3}\right.$, Is, C33), $24.9\left(\mathrm{CH}_{3}, \mathrm{Is}\right.$, C34), $22.3\left(\mathrm{CH}_{3}\right.$, Is, $\left.\mathrm{C} 29\right), 18.8-18.2$ (m, $\mathrm{CH}_{3}$, Duphos, $\left.\mathrm{C} 20\right)$, 16.3-15.9 (m, $\mathrm{CH}_{3}$, Duphos, C12), 15.5 (d, J = 15, $\mathrm{CH}_{3}$, Duphos, C24), 15.1 (d, J = 13, $\mathrm{CH}_{3}$, Duphos, C11), 12.4 (broad, $\mathrm{P}-\mathrm{CH}_{3}, \mathrm{C} 40$ ). 
Table S1. ${ }^{1} \mathrm{H}$ and ${ }^{13} \mathrm{C}$ NMR Assignments for the Major Diastereomer of $\mathrm{Pt}((\mathrm{R}, \mathrm{R})-\mathrm{Me}-$ Duphos $)(\mathrm{Ph})(\mathrm{PMeIs})$ in $\mathrm{THF}-\mathrm{d}_{8}$ at $-60^{\circ} \mathrm{C}$

\begin{tabular}{|c|c|c|c|c|}
\hline Proton & $\delta\left({ }^{1} \mathbf{H}\right)$ & $\delta\left({ }^{15} \mathrm{C}\right)$ & Assignment & $\delta\left({ }^{15} \mathrm{C}\right)$ (quat) \\
\hline a (H15) & $7.87-7.86$ & $134.5-134.0$ & $\mathrm{Ar} \mathrm{Du}$ & $163.0(\mathrm{C} 1)$ \\
\hline $\mathbf{b}(\mathrm{H} 16 / 6)$ & $7.78-7.72$ & $135.2-134.6 / 140.2$ & $\mathrm{Ar} \mathrm{Du}+o-\mathrm{Ph}$ & $155.6(\mathrm{C} 31)$ \\
\hline $\mathbf{c}(\mathrm{H} 14 / 17)$ & $7.56-7.55$ & $131.3 / 131.9$ & $\mathrm{Ar} \mathrm{Du}$ & $154.0(\mathrm{C} 25)$ \\
\hline d $(\mathrm{H} 2)$ & 7.25 & 137.9 & $o-\mathrm{Ph}$ & $148.1(\mathrm{C} 26 / \mathrm{C} 36)$ \\
\hline $\mathbf{e}(\mathrm{H} 5)$ & 7.07 & 128.3 & $m-\mathrm{Ph}$ & $147.2-146.6(\mathrm{C} 18)$ \\
\hline $\mathbf{f}(\mathrm{H} 30)$ & 6.99 & 121.8 & Is $m$ & $144.1-143.3(\mathrm{C} 13)$ \\
\hline $\mathbf{g}(\mathrm{H} 3)$ & 7.00 & 128.1 & $m-\mathrm{Ph}$ & \\
\hline h (H35) & 6.99 & 120.0 & Is $m$ & \\
\hline $\mathbf{i}(\mathrm{H} 4)$ & 6.78 & 122.1 & $p$-Ph & \\
\hline $\mathbf{j}(\mathrm{H} 27)$ & $4.92-4.87$ & 33.4 & Is $\mathrm{CH}$ & \\
\hline $\mathbf{k}(\mathrm{H} 37)$ & $4.72-4.70$ & 35.1 & Is $\mathrm{CH}$ & \\
\hline I (H32) & $2.94-2.81$ & 35.5 & Is $\mathrm{CH}$ & \\
\hline $\mathbf{m}(\mathrm{H} 7 / 10)$ & $2.94-2.81$ & $32.9 / 42.4$ & Du CH & \\
\hline $\mathbf{n}(\mathrm{H} 19)$ & $2.44-2.39$ & $45.1-44.7$ & $\overline{\mathrm{Du} C H}$ & \\
\hline $\mathbf{0}$ & $1.99-1.77(4 \mathrm{H})$ & $38.1 / 36.9-35.7$ & $\mathrm{CH}_{2}$ & \\
\hline $\bar{p}$ & $1.64-1.53(3 \mathrm{H})$ & $38.1 / 36.9-35.7$ & $\mathrm{CH}_{2}$ & \\
\hline $\mathbf{q}(\mathrm{H} 20)$ & $1.49(3 \mathrm{H})$ & $18.8-18.2$ & $\mathrm{Du} \mathrm{Me}$ & \\
\hline $\mathbf{r}$ & $1.64-1.53(1 \mathrm{H})$ & $38.1 / 36.9-35.7$ & $\mathrm{CH}_{2}$ & \\
\hline $\mathbf{s}(\mathrm{H} 12)$ & $1.39(3 \mathrm{H})$ & $16.3-15.9$ & $\mathrm{Du} \mathrm{Me}$ & \\
\hline $\mathbf{t}(\mathrm{H} 29 / 38)$ & $1.36-1.29(6 \mathrm{H})$ & $22.3 / 27.3$ & Is Me & \\
\hline $\mathbf{u}(\mathrm{H} 39)$ & $1.23(3 \mathrm{H})$ & 28.1 & Is Me & \\
\hline $\mathbf{v}(\mathrm{H} 33 / 34)$ & $1.23(6 \mathrm{H})$ & $25.1 / 24.9$ & Is Me & \\
\hline $\mathbf{w}(\mathrm{H} 23)$ & $1.36-1.29(1 \mathrm{H})$ & 32.6 & $\overline{\mathrm{Du} \mathrm{CH}}$ & \\
\hline $\bar{x}(\mathrm{H} 28)$ & $1.03(3 \mathrm{H})$ & 28.3 & Is Me & \\
\hline $\mathbf{y}(\mathrm{H} 11)$ & \begin{tabular}{|l}
$0.77-0.73(3 \mathrm{H})$ \\
\end{tabular} & 15.1 & $\overline{\mathrm{Du} \mathrm{Me}}$ & \\
\hline $\mathbf{z}(\mathrm{H} 24)$ & $0.61(3 \mathrm{H})$ & 15.5 & $\mathrm{Du} \mathrm{Me}$ & \\
\hline aa $(\mathrm{H} 40)$ & $0.77-0.73(3 \mathrm{H})$ & 12.4 & $\mathrm{P}-\mathrm{Me}$ & \\
\hline
\end{tabular}


Chart S1 ${ }^{1} \mathrm{H}$ (black) and ${ }^{13} \mathrm{C}$ (red) NMR assignments for the major diastereomer of 4 (THF-d $\mathrm{d}_{8}$, $\left.-60{ }^{\circ} \mathrm{C}\right)$.
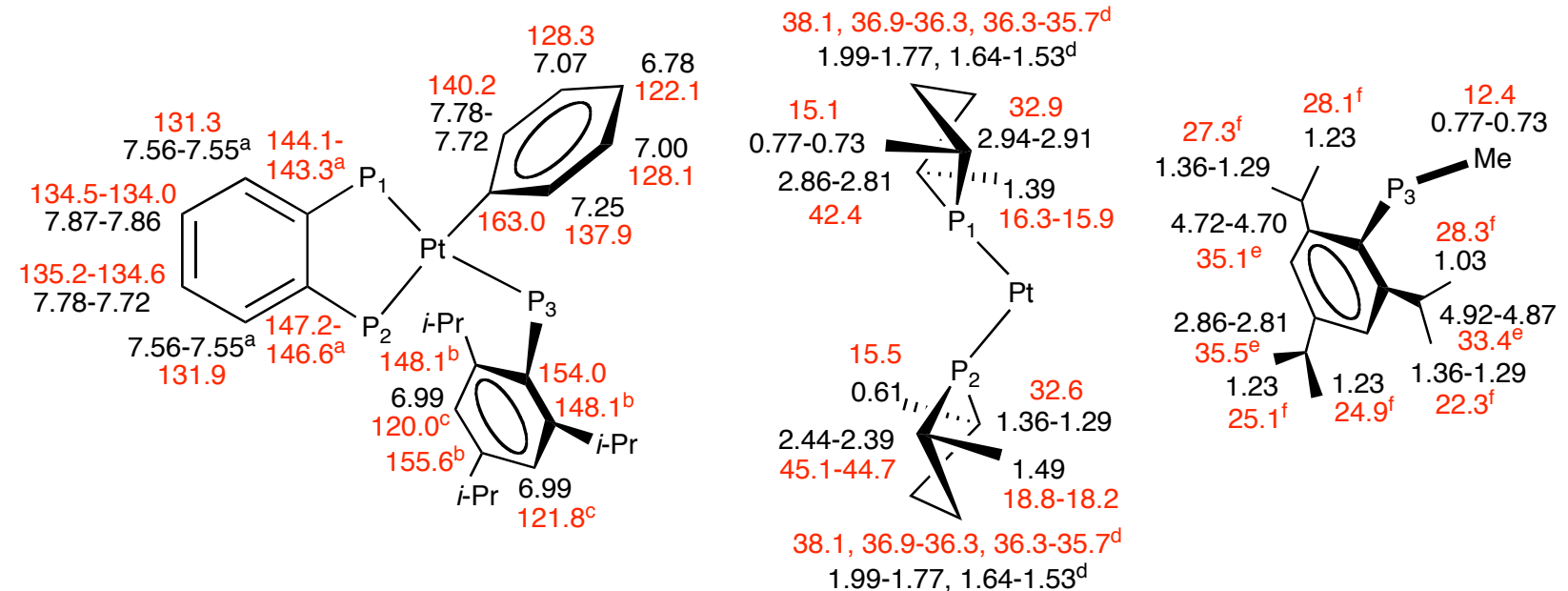

${ }^{a}$ Which Duphos quaternary $\mathrm{C}$ atom is adjacent to $\mathrm{P}_{1}$ (or $\mathrm{P}_{2}$ ) could not be determined; similarly, the $o$-Duphos Ar $\mathrm{CH}$ signals overlapped ${ }^{\mathrm{b}}$ Only two of the three expected ${ }^{13} \mathrm{C}$ NMR signals for the Is $o$ - and $p$-quaternary $\mathrm{C}$ atoms were observed; we can't tell the difference between the $o$ - and $p$ resonances ${ }^{\mathrm{c}} \mathrm{We}$ cannot tell which Is $m$-C is on which side of the ring because of the coincident ${ }^{1} \mathrm{H}$ NMR shifts of $\mathrm{H} 30$ and $\mathrm{H} 35 .{ }^{d}$ We could not differentiate the $\mathrm{CH}_{2}$ groups in the phospholane, or unambiguously assign their ${ }^{13} \mathrm{C}$ signals ${ }^{\mathrm{e}}$ The assignment of the $o-\mathrm{CH}(i$-Pr $){ }^{1} \mathrm{H}$ NMR peaks shown is based on their similar chemical shifts and NOE data ${ }^{f}$ Because of ${ }^{1} \mathrm{H}$ NMR peak overlap of the $\mathrm{Me}\left(i\right.$-Pr) signals, definitive assignment of the corresponding ${ }^{13} \mathrm{C}$ NMR signals was not possible. The assignments shown are consistent with correlations between the $i$-Pr $\mathrm{CH}$ and Me signals, but unambiguous assignment of individual peaks to $o$ - or $p$-positions was not possible. 


\section{${ }^{1} \mathrm{H}-{ }^{1} \mathrm{H}$ NOESY-based Stereochemistry Assignment}

1. NOEs between the Duphos CHMe groups (of known absolute configuration in the (R,R)ligand) and the Duphos Ar backbone were used to determine the positions of the two CHMe groups in each phospholane.

a. For the P2 phospholane, NOEs from $\mathrm{H} 19$ and the methyl group, $\mathrm{C} 24$, to the ring hydrogens H17 and H16 established their assignments, and, by extension, that of their CHMe neighbors, C20 and H23, respectively. An NOE was also observed from methyl C20 to H17, but overlap of the H23 signal with other resonances prevented confident assignment of its NOEs.

Figure S2. NOEs between the P2 phospholane and the Duphos Ar ring and resulting assignments.
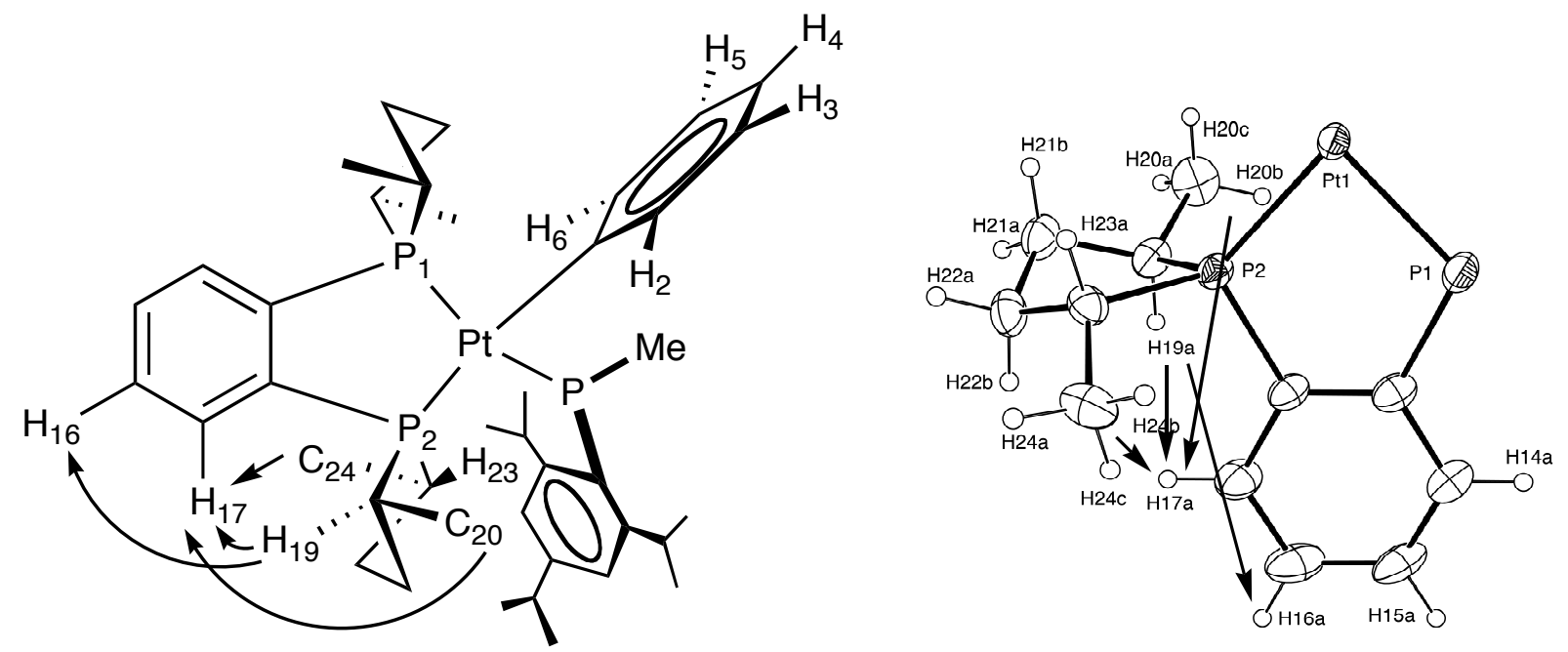

These observations are consistent with the following distances in the solid-state structure (in $\AA$, estimated using the calculated hydrogen atom positions): H19-H16 4.325, H19-H17 2.435, H24H17 3.237 (ave), H20-H17 4.766 (ave). 
b. For the P1 phospholane, NOEs from $\mathrm{H} 10$ and the methyl group, $\mathrm{C} 11$, to the ring hydrogens H14 and H15 established their assignments, and, by extension, that of their CHMe neighbors, C12 and $\mathrm{H} 7$, respectively. NOEs were also observed from $\mathrm{H} 7$ and the $\mathrm{C} 12$ methyl group to $\mathrm{H} 14$.

Figure S3. NOEs between the P1 phospholane and the Duphos Ar ring and resulting assignments.
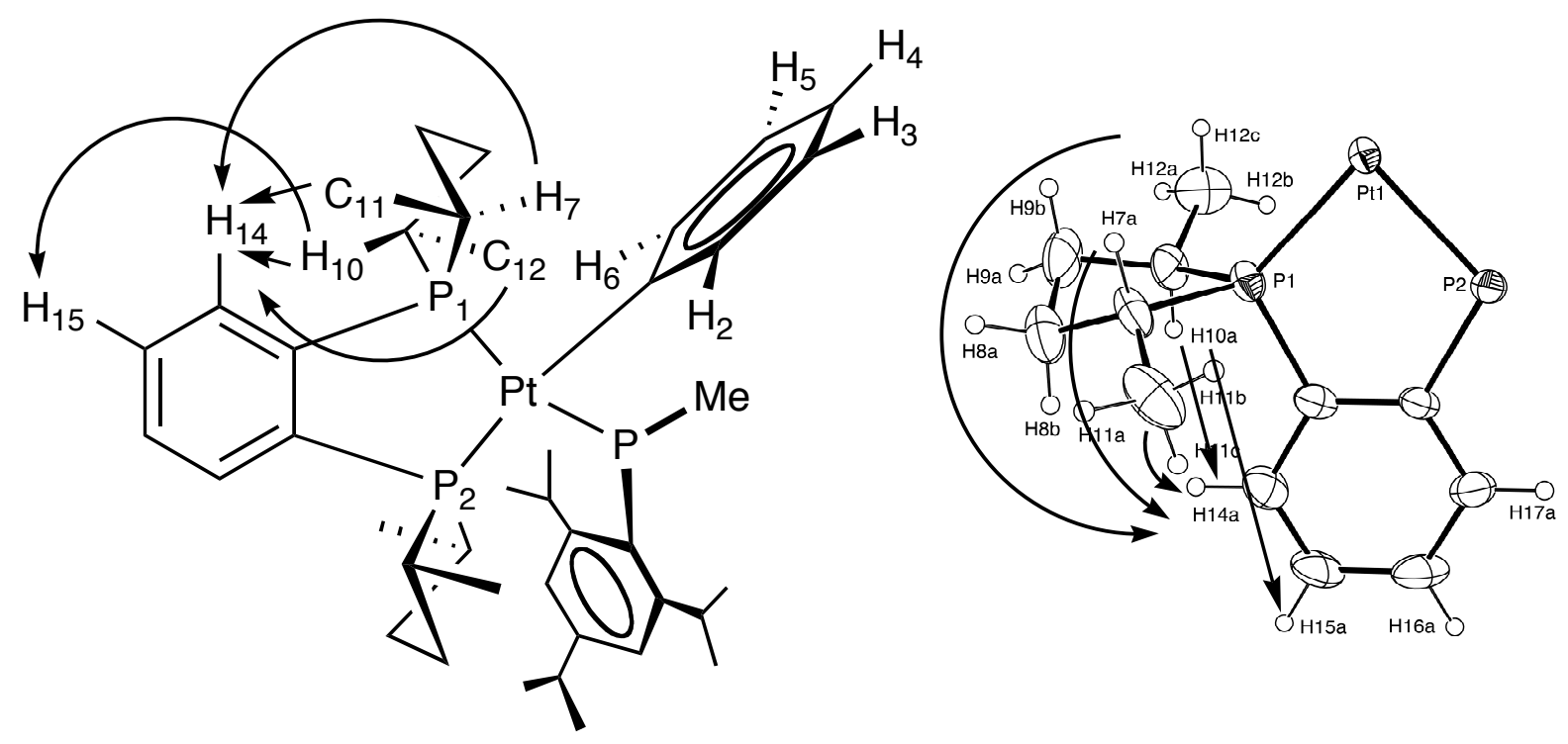

These observations are consistent with the following distances in the solid-state structure (in $\AA$, estimated using the calculated hydrogen atom positions): H10-H15 4.358, H10-H14 2.430, H11H14 3.596 (ave), H7-H14 4.155, H12-H14 4.739 (ave). 
2. NOEs between the P1 phospholane CHMe signals and the Pt-Ph ones were used to determine the conformation of the Pt-Ph ring.

In the solid-state structure, the Pt-Ph group was perpendicular to the Pt square plane, with $\mathrm{H} 2$ and $\mathrm{H} 3$ above the plane, and $\mathrm{H} 5$ and $\mathrm{H} 6$ below it. The observed NOEs from $\mathrm{H} 7$ to $\mathrm{H} 2$ and $\mathrm{H} 3$, and from methyl $\mathrm{C} 12$ to $\mathrm{H} 6$ and $\mathrm{H} 5$ suggested that this conformation was retained in solution.

Figure S4. NOEs between the P1 phospholane and the Pt-Ph hydrogens and resulting assignments.
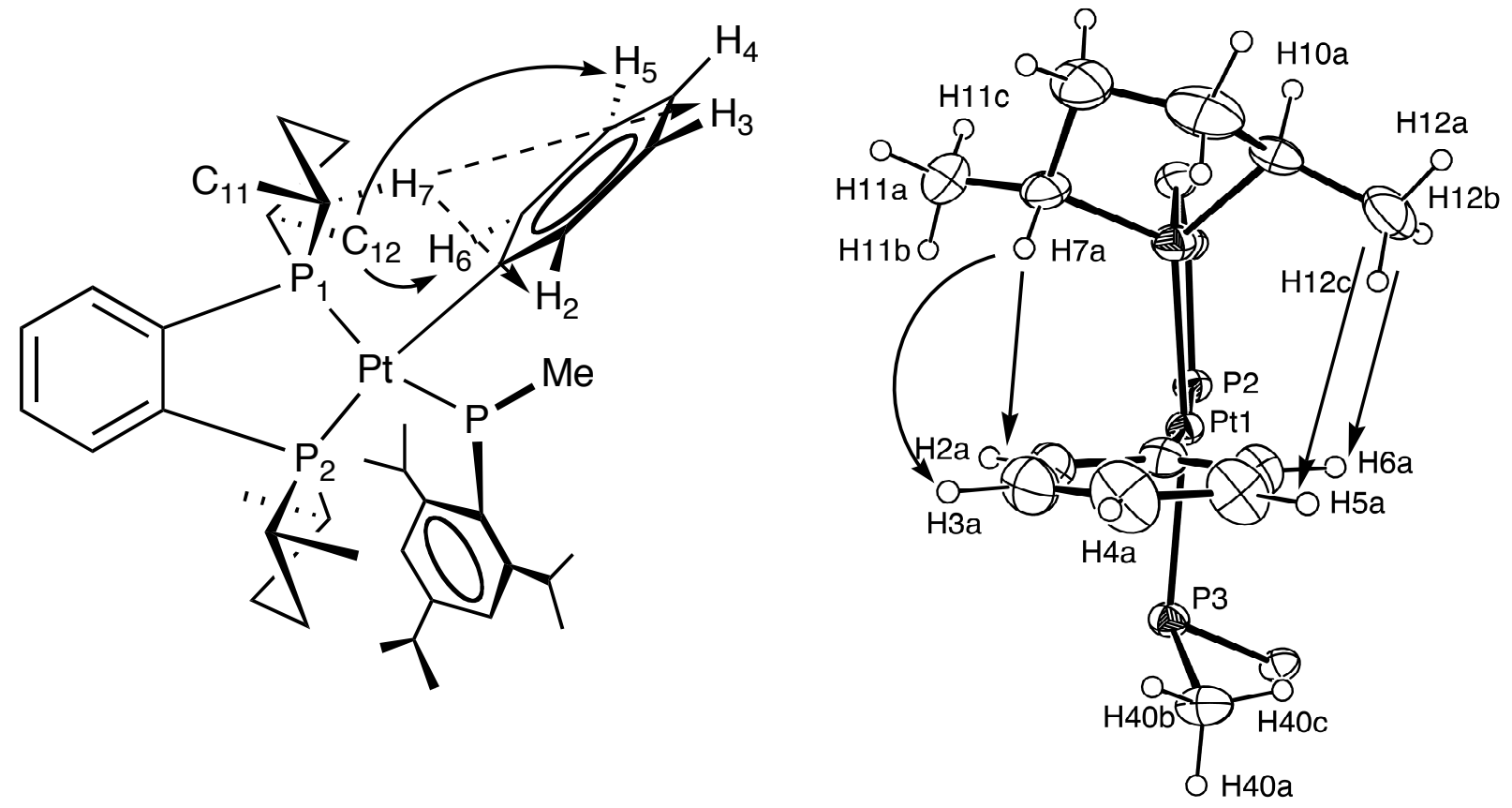

These observations are consistent with the following distances in the solid-state structure (in $\AA$, estimated using the calculated hydrogen atom positions): $\mathrm{H} 7-\mathrm{H} 2$ 2.471, H7-H3 3.720, H12-H6 3.168 (ave), and H12-H5 4.725 (ave).

This information on the structure and conformation of the $\operatorname{Pt}((\mathrm{R}, \mathrm{R})-\mathrm{Me}-\mathrm{Duphos})(\mathrm{Ph})$ fragment was then used to investigate the absolute configuration of the phosphido ligand. Chart S2 shows 
three different ways to arrange the Me, Is and Pt substituents and the lone pair about the pyramidal P center. $R_{P}-\mathbf{4}$ corresponds to the solid-state structure (Figure S1). The opposite stereochemistry $\left(S_{P}-\mathbf{4}\right)$ was observed in the crystal structure of 4-i-Pr. Finally, rotation of $S_{P}-\mathbf{4}$ about the Pt-P bond would yield diastereomer $S_{P}-\mathbf{4}^{\prime}$, which is related to the solid-state structure of cations $\mathbf{8}$ and $\mathbf{1 2}$.

Chart S2. Possible Phosphido Configurations in the Major Diastereomer of $\mathbf{4}$, with Solid-State Models

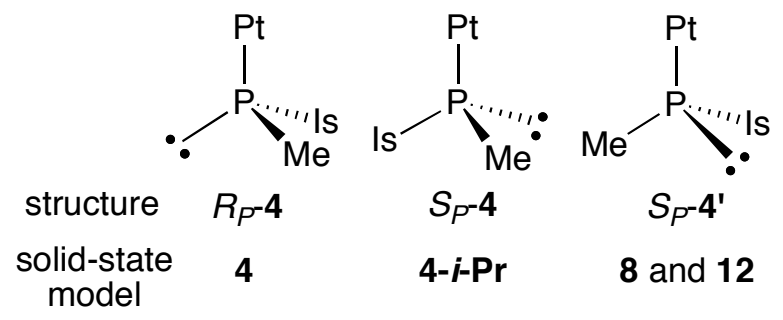

3. NOEs between the PMeIs group and the $\mathrm{Pt}((\mathrm{R}, \mathrm{R})-\mathrm{Me}-\mathrm{Duphos})(\mathrm{Ph})$ fragment were used to differentiate these structures. First, several P-Me-to-Pt-Ph NOEs were observed (Figure S5), but since the PMe (C40) and Duphos Me (C11) ${ }^{1} \mathrm{H}$ NMR signals overlapped, assignment was not straightforward. However, estimating the $\mathrm{H}-\mathrm{H}$ distances (Pt-Ph to P-Me and Pt-Ph to Duphos $\mathrm{Me}$ ) from the crystal structure of $R_{P}-\mathbf{4}$ enabled assignment of NOEs to one or both of the methyl groups (Table S2 and Figure S5). 
Table S2. Calculated average distances $(\AA)$ between the Pt-Ph hydrogens and P-Me (C40) and Duphos Me (C11) hydrogens in the structure of $\mathbf{4}$ and the resulting assignment of the observed Pt$\mathrm{Ph} / \mathrm{Me}$ NOEs.

\begin{tabular}{|l|l|l|l|}
\hline Pt-Ph Hydrogens & $\begin{array}{l}\text { d(H-H) P-Me } \\
\text { (C40) (ave) }\end{array}$ & $\begin{array}{l}\text { d(H-H) CHMe } \\
(\mathbf{C 1 1} \text { (ave) }\end{array}$ & NOE assignment \\
\hline H2a & 4.099 & 4.001 & both \\
\hline H3a & 4.851 & 5.583 & both \\
\hline H4a & 5.053 & 7.168 & P-Me \\
\hline H5a & 4.400 & 7.547 & P-Me \\
\hline H6a & 3.532 & 6.495 & P-Me \\
\hline
\end{tabular}

Figure S5. Pt-Ph/P-Me NOEs and resulting assignments. ${ }^{a}$
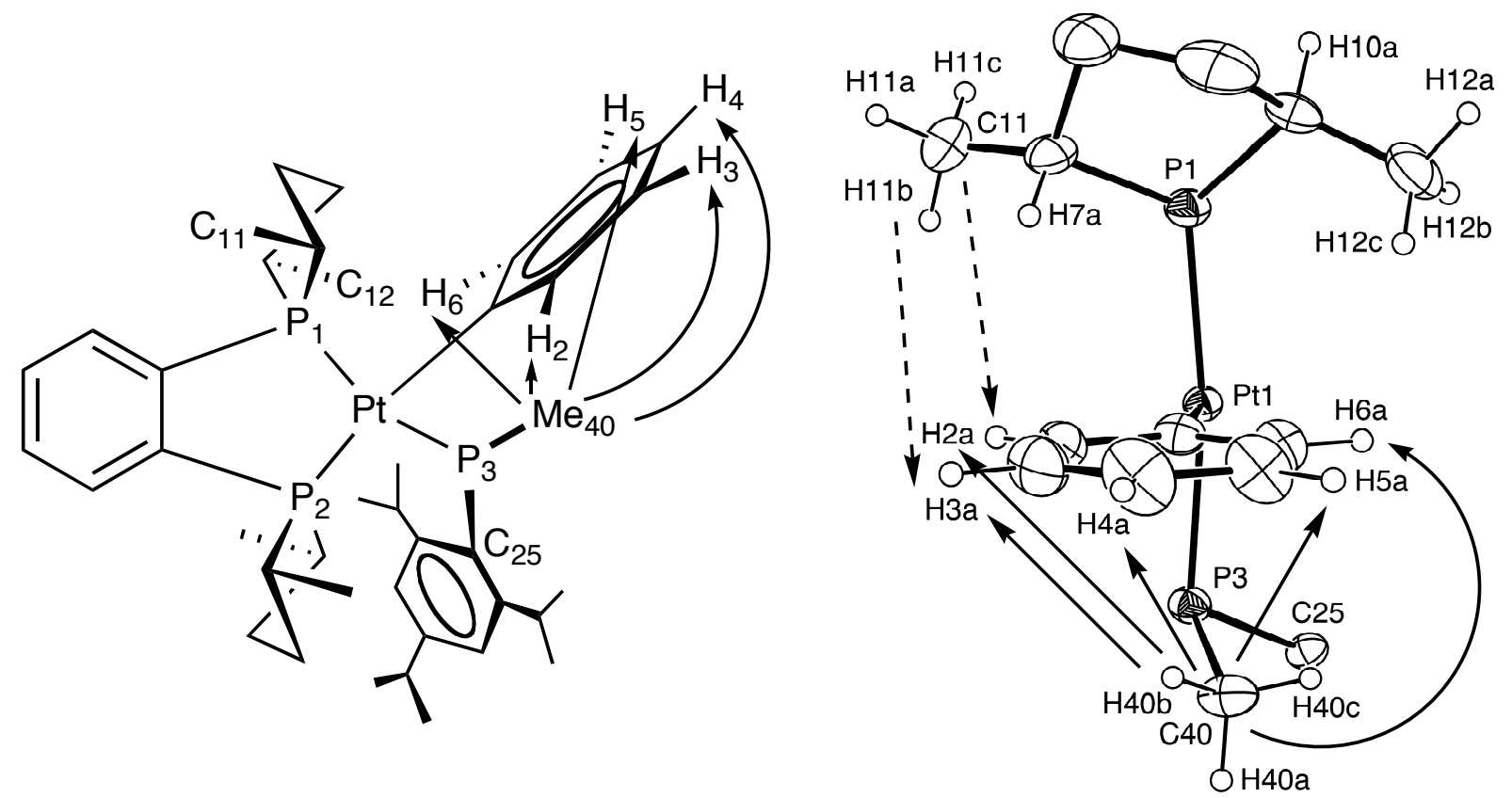

${ }^{a}$ For clarity, NOEs from the $\mathrm{C} 11 \mathrm{Me}$ group to the Pt-Ph hydrogens are not shown in the left-hand panel (see discussion above). Arrows in the right-hand panel (a portion of the ORTEP diagram in Figure S1) show NOEs from both the P-Me (C40, solid arrows) and the C11 Me (dashed arrows) to $\mathrm{H} 2$ and $\mathrm{H} 3$. As discussed above, however, we can't tell which of the two methyl groups makes a bigger contribution to these NOEs. 
4. NOEs between the Is isopropyl hydrogens and the $\mathrm{Pt}-\mathrm{Ph}$ hydrogens provided information on the conformation of the isityl group. Despite possible rotations about the P-C and the C-C $(i-\mathrm{Pr})$ bonds, the NOE data were consistent with the solid-state structure; the isopropyl methine H37 $(\delta$ 4.72-4.70) showed the NOEs illustrated in Figure S6, consistent with the following distances in the solid-state structure (in $\AA$, estimated using the calculated hydrogen atom positions): H37-H6 2.393, H37-H5 4.495. These observations are consistent with the relative Me and Is positions shown (and hence with the $R_{P}$ stereochemistry).

Figure S6. NOEs observed between H37 and the Pt-Ph hydrogens.
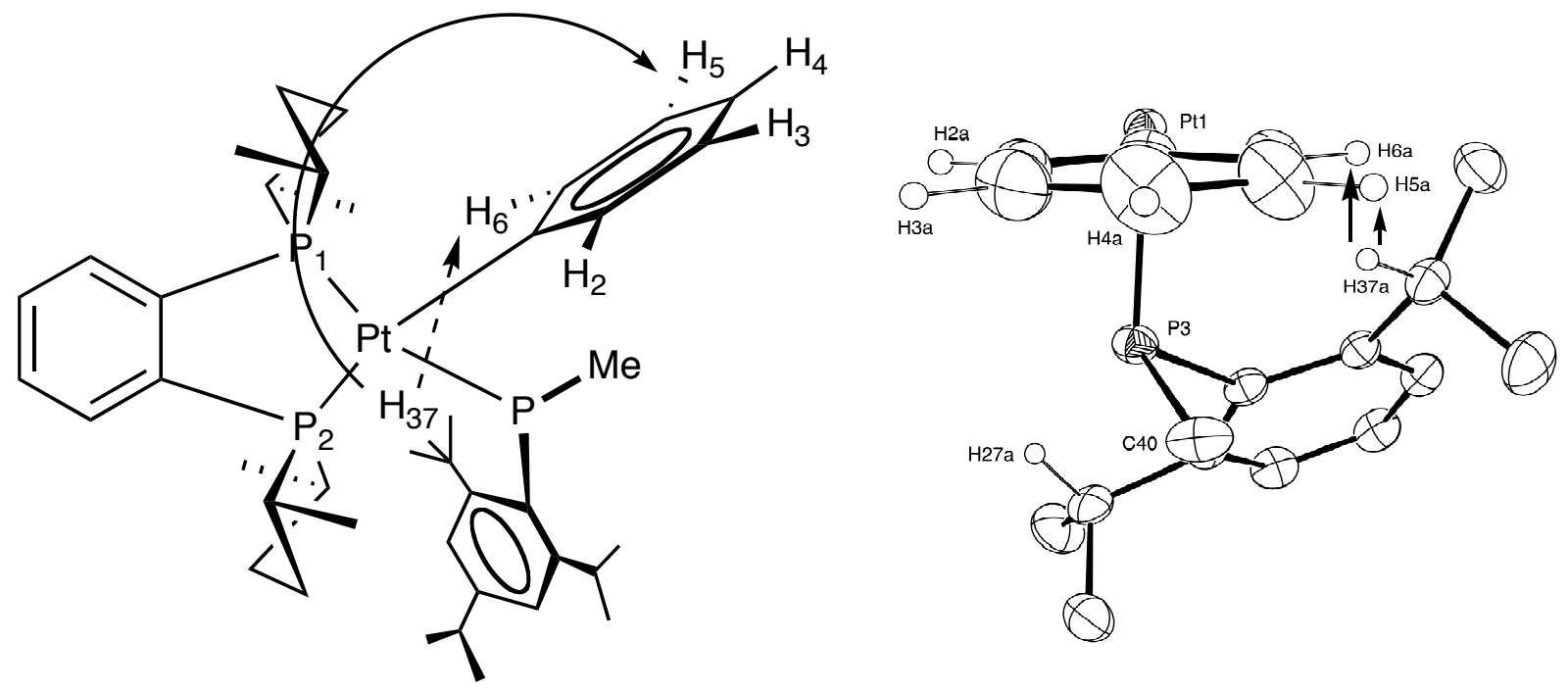

5. Additional NOEs between hydrogens in the Is group and the phospholane and $\mathrm{Pt}-\mathrm{Ph}$ groups were also consistent with the solid-state structure. 
Figure S7. NOEs between the Is group and the P2 phospholane (left) and selected hydrogens in the Pt-Ph (H6) and P1 phospholane (H12) groups (right).
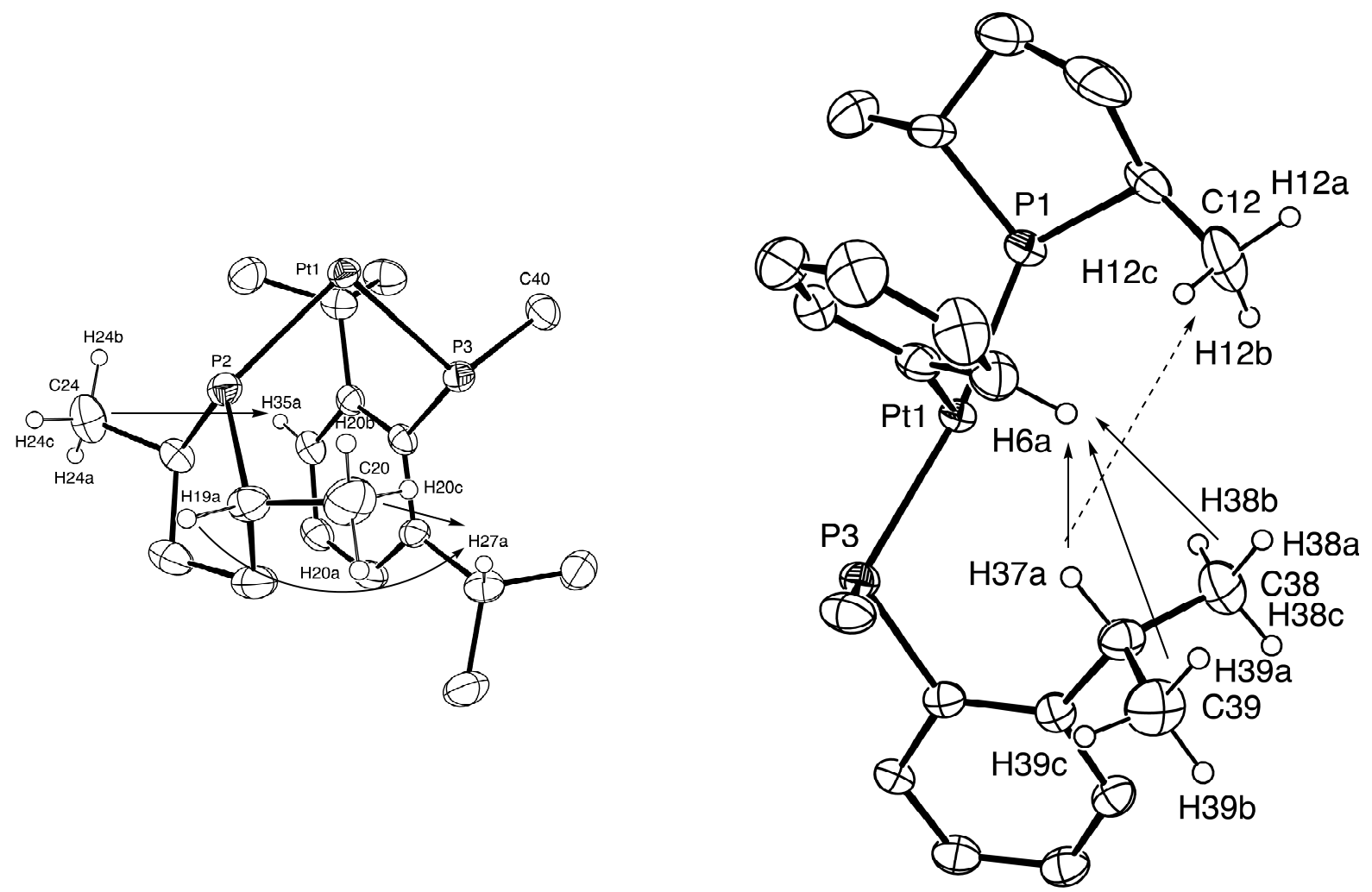

Figure S7 (left) shows NOEs from the P2 phospholane hydrogens H19 and H20 to the Is hydrogen $\mathrm{H} 27$, and an NOE from phospholane H24 to Is H35. These observations are consistent with the distances $(\AA)$ estimated using the calculated H positions from the solid-state structure: H24-H35 4.501 (ave), H20-H27 3.610 (ave), and H19-H27 4.804. Figure S7 (right) shows (solid arrows) NOEs to H6 from H37 (also shown in Figure S6), as well as from the adjacent methyl groups $\mathrm{H} 38$ and H39, plus an NOE observed between $\mathrm{H} 37$ and H12 in the P1 phospholane (dashed arrow). These observations are consistent with the distances $(\AA)$ estimated using the calculated $\mathrm{H}$ positions from the solid-state structure: H6-H38 3.860 (ave), H6-H39 3.790 (ave), H37-H12 4.173 (ave). 
Ideally, determination of the solution structures of both diastereomers of $\mathbf{4}$, and comparison of the data, would enable definitive assignment of the absolute configuration. However, because of the thermodynamic bias for one diastereomer of $\mathbf{4}$, presumed to have $R_{P}$ configuration, detailed NMR studies of the minor $\left(S_{P}\right)$ isomer were not possible. ${ }^{3}$

The crystal structure of the analogue $S_{P}-\mathrm{Pt}((\mathrm{R}, \mathrm{R})-i-\mathrm{Pr}-\mathrm{Duphos})(\mathrm{Ph})(\mathrm{PMeIs})(4-i-\mathrm{Pr})$ provided a model for $S_{P}-\mathbf{4}^{2}$ The $\mathrm{Pt}(\mathrm{Ph})(\mathrm{PMeIs})$ fragments in the solid-state structures of $\mathbf{4}$ and $\mathbf{4 - i}-\mathbf{P r}$ adopted similar conformations. As a result of the opposite absolute configurations, however, the PMeIs group was found on different sides of the Pt-Ph ring in 4 and 4-i-Pr (Figure S8). The position of the P-Me groups ( $\mathrm{C} 40$ and $\mathrm{C} 1$, respectively) changed little in these structures; the estimated P-Meto-Pt-Ph H-H distances using 4-i-Pr as a model for $S_{P^{-}} \mathbf{4}$ (as in Table S2; see Table S3), were also consistent with the observed NOEs.

Figure S8. Partial ORTEP diagrams ( $p$ - $i$-Pr groups omitted) for the complexes $R_{P}-\mathrm{Pt}((\mathrm{R}, \mathrm{R})-\mathrm{Me}-$ Duphos)(Ph)(PMeIs) (4) and $S_{P}-\mathrm{Pt}((\mathrm{R}, \mathrm{R})-i$-Pr-Duphos)(Ph)(PMeIs) (4-i-Pr, one of the two independent molecules in the unit cell), illustrating close contacts between ortho $i$-Pr $\mathrm{CH}$ groups H37 (in 4) and H8 (in 4-i-Pr) and the Pt-Ph ring. ${ }^{\text {a }}$
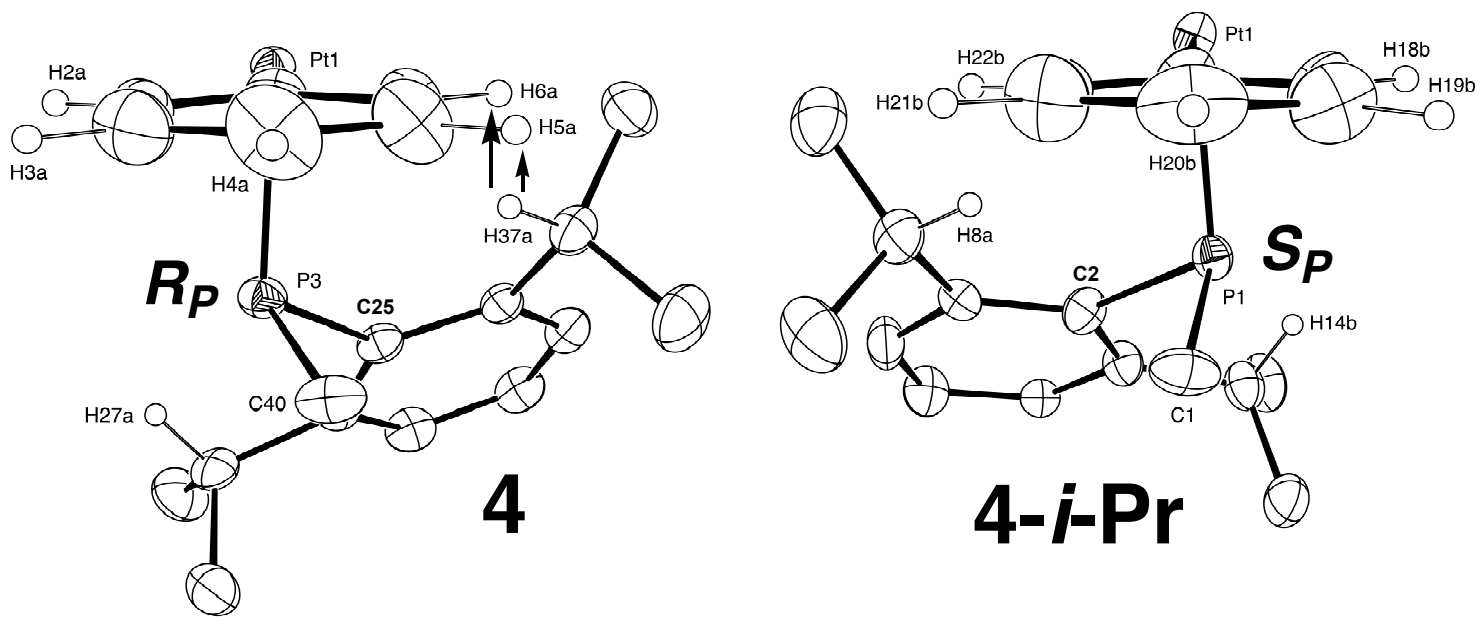

${ }^{a}$ The arrows on the left show NOEs between $i$-Pr CH H37 and Pt-Ph hydrogens H5 and H6, as in Figure S6. 
Table S3. Calculated average distances $(\AA)$ between the $\mathrm{Pt}-\mathrm{Ph}$ hydrogens and the $\mathrm{P}-\mathrm{Me}(\mathrm{C} 40$, in 4, or $\mathrm{C} 1$ in $\mathbf{4 - i - P r})$ in the structures of $\mathbf{4}$ and $\mathbf{4 - i - P r}{ }^{\mathrm{a}}$

\begin{tabular}{|l|l|l|l|}
\hline Pt-Ph (4) & $\begin{array}{l}\text { d(H-H) } \\
(\mathbf{C 4 0}, \text { P-Me })\end{array}$ & Pt-Ph (4-i-Pr) & $\begin{array}{l}\text { d(H-H) } \\
(\mathbf{C 1}, \text { P-Me })\end{array}$ \\
\hline H2a $(o)$ & 4.099 & H22a $(o)$ & 3.694 \\
\hline H3a $(m)$ & 4.851 & H21a $(m)$ & 4.600 \\
\hline H4a $(p)$ & 5.053 & H20a $(p)$ & 5.090 \\
\hline H5a $(m)$ & 4.400 & H19a $(m)$ & 4.741 \\
\hline H6a $(o)$ & 3.532 & H18a $(o)$ & 3.906 \\
\hline
\end{tabular}

${ }^{a}$ For 4-i-Pr, average distances for the two independent molecules are reported; $\mathrm{H}$ atoms were placed in calculated positions.

Table S4. Selected Estimated Solid-state H-H and H-C Distances (Å) in the Complexes $R_{P^{-}}$ $\operatorname{Pt}((\mathrm{R}, \mathrm{R})-\mathrm{Me}-\mathrm{Duphos})(\mathrm{Ph})(\mathrm{PMeIs})(4)$ and $S_{P}-\mathrm{Pt}((\mathrm{R}, \mathrm{R})-i-\mathrm{Pr}-\mathrm{Duphos})(\mathrm{Ph})(\mathrm{PMeIs})\left(\right.$ 4-i-Pr). ${ }^{\mathrm{a}}$

\begin{tabular}{|c|c|c|c|c|c|c|c|}
\hline \begin{tabular}{|l|l|} 
Pt-Ph (4) \\
\end{tabular} & $\begin{array}{l}\mathbf{d}(\mathbf{H}-\mathbf{H}) \\
(\mathbf{H 3 3 7})\end{array}$ & $\begin{array}{l}\mathbf{d}(\mathbf{C}-\mathrm{H}) \\
(\mathbf{C 4 0}, \mathrm{Me})\end{array}$ & $\begin{array}{l}\mathbf{d}(\mathrm{C}-\mathrm{H}) \\
(\mathbf{C} 25, \mathrm{Is})\end{array}$ & \begin{tabular}{|l|} 
Pt-Ph \\
(4-i-Pr)
\end{tabular} & $\begin{array}{l}\mathbf{d}(\mathbf{H}-\mathbf{H}) \\
(\mathbf{H 8})\end{array}$ & $\begin{array}{l}\mathbf{d}(\mathrm{C}-\mathrm{H}) \\
(\mathrm{C} 1, \mathrm{Me})\end{array}$ & $\begin{array}{l}\text { d(C-H) } \\
(\mathrm{C} 2, \mathrm{Is})\end{array}$ \\
\hline $\mathrm{H} 2 \mathrm{a}(o)$ & 4.895 & 3.913 & 5.507 & \begin{tabular}{|l|}
$\mathrm{H} 22(o)$ \\
\end{tabular} & 2.245 & 3.607 & 4.643 \\
\hline H3a $(m)$ & 6.166 & 4.840 & 7.165 & H21 (m) & 4.290 & 4.669 & 6.515 \\
\hline $\mathrm{H} 4 \mathrm{a}(p)$ & 6.039 & 5.140 & 7.655 & \begin{tabular}{|l|}
$\mathrm{H} 20(p)$ \\
\end{tabular} & 5.860 & 5.190 & 7.598 \\
\hline H5a $(m)$ & 4.495 & 4.478 & 6.609 & \begin{tabular}{|l}
$\mathrm{H} 19(\mathrm{~m})$ \\
\end{tabular} & 6.037 & 4.764 & 7.178 \\
\hline H6a $(o)$ & 2.393 & 3.453 & 4.738 & \begin{tabular}{|l|}
$\mathrm{H} 18(o)$ \\
\end{tabular} & 4.816 & 3.754 & 5.546 \\
\hline
\end{tabular}

${ }^{\text {a }}$ Distances were estimated using calculated hydrogen atom positions; average values for the two independent molecules are reported for 4-i-Pr. Highlighted distances in bold for $\mathbf{4}$ correspond to observed NOEs, while highlighted distances in bold for 4-i-Pr correspond to predicted NOEs.

However, the position of the P-Is groups (only the ipso carbons are shown in Figure S8) differed significantly in 4 and 4-i-Pr. Although the argument requires several assumptions, a combination 
of the estimated $\mathrm{H}-\mathrm{H}$ distances between the $o-i$-Pr methine hydrogens $\mathrm{H} 37$ (in 4) and $\mathrm{H} 8$ (in 4-iPr) and the Pt-Ph ring (Table S4) and the NOE observations may be used to differentiate structures 4 and 4-i-Pr and the associated P stereochemistries.

The observed NOEs for 4 from H37 to H6 and H5 (Figures S6-S8) were consistent with the estimated solid-state distances, highlighted in yellow in Table S4. Hypothetical $S_{P^{-}} \mathbf{4}$ (if 4-i-Pr is a good model for it) would be expected to show NOEs from H37 to H2, H3, and H6, but not to H4 and H5 (Table S4, highlighted in gray for H8, the analogue of H37 in 4-i-Pr). Although it is dangerous to make arguments based on negative observations, ${ }^{4}$ the lack of NOEs between H37 and $\mathrm{H} 2$ or H3, combined with the observed $\mathrm{H} 37-\mathrm{H} 5 \mathrm{NOE}$, is consistent with $R_{P}-\mathbf{4}$ and not with $S_{P}-\mathbf{4}$ (as modeled by $\left.S_{P}-\mathbf{4 - i}-\mathbf{P r}\right)$.

It is difficult to rule out another possible structure $\left(S_{P}-\mathbf{4}^{\prime}\right)$, derived from $R_{P}-\mathbf{4}$ by interchanging the $\mathrm{P}-\mathrm{Me}$ and the lone pair, which would require minimal motion and be consistent with observed NOEs to the Is group (Figure S8). However, comparison to the crystal structures of analogous complexes provided some evidence against this structure. Table S4 includes estimated H-C distances from the Pt-Ph hydrogens to the P-C carbons in $\mathbf{4}$ and 4-i-Pr. The position of Is ipso carbon $\mathrm{C} 2$ in 4-i-Pr is a model for the P-Me carbon in hypothetical $S_{P}-\mathbf{4}$ ' (Figure S8). The significantly longer estimated distances to it, in comparison to those to the real Me group (C1) are potentially inconsistent with the NOE observations. A similar trend is apparent for the $\mathrm{C} 40-\mathrm{H}(\mathrm{Ph})$ and $\mathrm{C} 25-\mathrm{H}(\mathrm{Ph})$ distances in 4 (Table S4).

Similarly, Figure S9 shows portions of the solid-state structures of $\mathbf{4}$ and its protonated (12) and alkylated (8) analogues. ${ }^{2}$ The P-H hydrogen (H3) in 12 provided another model for the position of the P-Me in hypothetical $S_{P}-4$ '. The calculated position of $\mathrm{H} 3$ was further from the $\mathrm{Pt}-\mathrm{Ph}$ ring than was the P-Me carbon (C40, see Table S5), potentially inconsistent with the observed NOEs of Figure S5. Rotation about the Pt-P bond in $\mathbf{8}-\mathbf{B F}_{\mathbf{4}}$ places the benzyl group (C34) in a position distinct from those of other P substituents in 4, 4-i-Pr, and 12. Here too C34 was further from the $\mathrm{Pt}-\mathrm{Ph}$ ring than the $\mathrm{P}-\mathrm{Me}$ carbon (C41, see Table S6). 
Figure S9. Partial ORTEP diagrams of the complexes $\mathrm{Pt}((\mathrm{R}, \mathrm{R})-\mathrm{Me}-\mathrm{Duphos})(\mathrm{Ph})(\mathrm{PMeIs})$ (4), $[\mathrm{Pt}((\mathrm{R}, \mathrm{R})-\mathrm{Me}-\mathrm{Duphos})(\mathrm{Ph})(\mathrm{PHMe}(\mathrm{Is}))]\left[\mathrm{BF}_{4}\right] \quad\left(\mathbf{1} \quad \mathbf{2}-\mathbf{B} \mathbf{F}_{\mathbf{4}}\right) \quad$ a n d $\quad[\mathrm{Pt}((\mathrm{R}, \mathrm{R})-\mathrm{Me}-$ Duphos $\left.)(\mathrm{Ph})\left(\mathrm{PMeIs}\left(\mathrm{CH}_{2} \mathrm{Ph}\right)\right)\right]\left[\mathrm{BF}_{4}\right]\left(\mathbf{8}-\mathbf{B F}_{4}\right)^{2}$
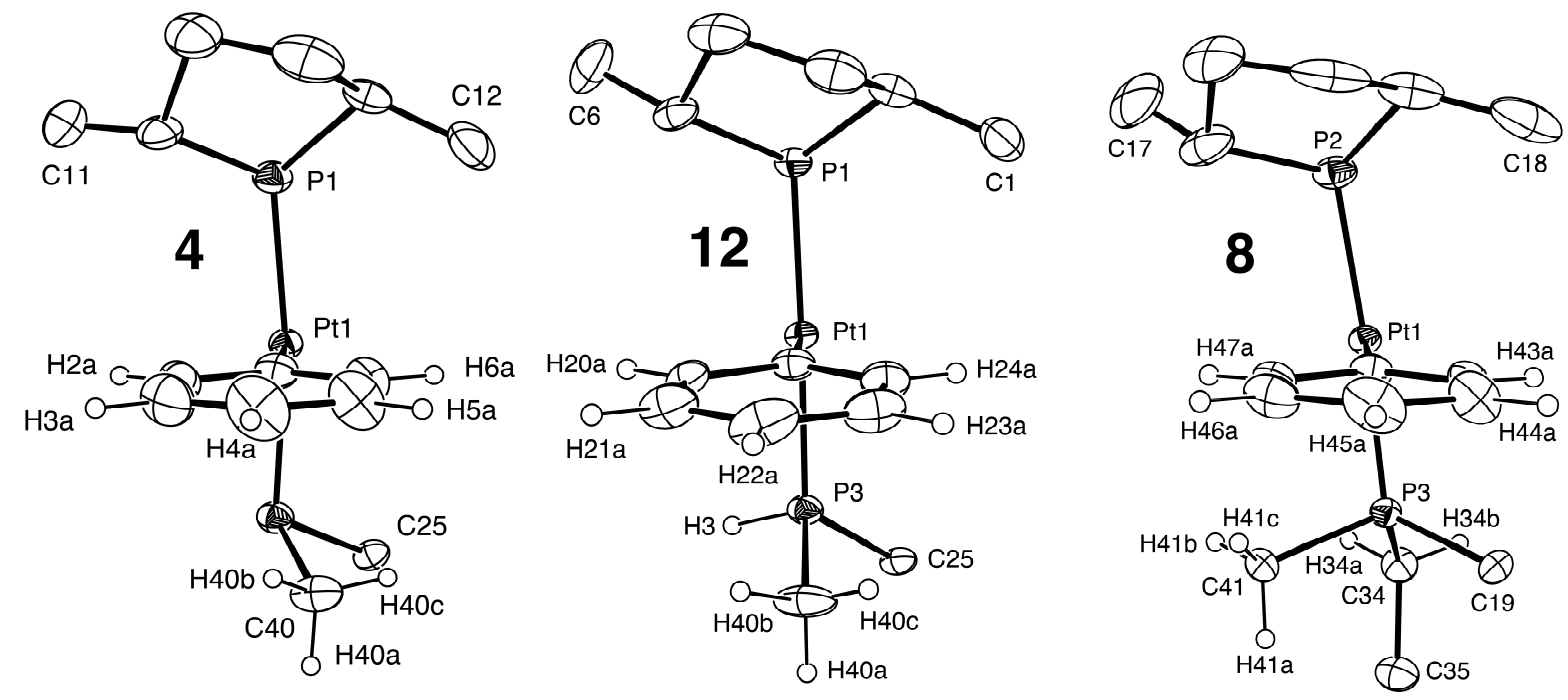

To enable comparison between the positions of the $\mathrm{P}-\mathrm{H}$ and $\mathrm{P}-\mathrm{Me}$ groups in 12, Table S5 compares calculated distances between the P-H hydrogen (H3), the Me carbon C40 and the ipso Is carbon $\mathrm{C} 25$ to the Pt-Ph hydrogens. Of these three substituents, the P-Me carbon showed the shortest estimated distances to the $\mathrm{Pt}-\mathrm{Ph}$ ring. (This comparison is necessarily a crude one because of the differing $\mathrm{P}-\mathrm{H}$ and $\mathrm{P}-\mathrm{C}$ distances, as well as the geometrical changes on protonation of 4 to yield 12). 
Table S5. Estimated distances ( $\mathrm{A})$ in the structure of $[\mathrm{Pt}((\mathrm{R}, \mathrm{R})-\mathrm{Me}-$ Duphos $)(\mathrm{Ph})(\mathrm{PHMe}(\mathrm{Is}))]\left[\mathrm{BF}_{4}\right](\mathbf{1 2})$. Hydrogen atoms were placed in calculated positions. ${ }^{2}$

\begin{tabular}{|l|l|l|l|}
\hline $\begin{array}{l}\text { Pt-Ph } \\
\text { Hydrogens }\end{array}$ & $\begin{array}{l}\text { d(H-C) to C40 } \\
\text { (P-Me) (ave) }\end{array}$ & $\begin{array}{l}\text { d(H-H) to } \\
\text { H3 (PH) }\end{array}$ & $\begin{array}{l}\text { d(H-C) to } \\
\text { C25 (Is) }\end{array}$ \\
\hline H20a $(o)$ & 3.764 & 3.982 & 5.554 \\
\hline H21a $(m)$ & 4.673 & 5.811 & 7.137 \\
\hline H22a $(p)$ & 5.021 & 6.873 & 7.529 \\
\hline H23a $(m)$ & 4.560 & 6.525 & 6.493 \\
\hline H24a $(o)$ & 3.664 & 4.985 & 4.713 \\
\hline
\end{tabular}

\begin{tabular}{l} 
Table S6 shows similar data for the benzylated complex $[\mathrm{Pt}((\mathrm{R}, \mathrm{R})-\mathrm{Me}-$ \\
\hline
\end{tabular} Duphos $\left.)(\mathrm{Ph})\left(\mathrm{PMeIs}\left(\mathrm{CH}_{2} \mathrm{Ph}\right)\right)\right]\left[\mathrm{BF}_{4}\right](\mathbf{8})$. Again, the closest calculated approach to the ring is for the P-Me group.

Table S6. Estimated $\mathrm{H}-\mathrm{H}$ distances $(\AA)$ between $\mathrm{P}-\mathrm{Me}(\mathrm{H} 41 \mathrm{a}-\mathrm{c})$ and $\mathrm{P}-\mathrm{CH}_{2} \mathrm{Ph}$ (H34a-b) hydrogens and the Pt-Ph hydrogens (H43a-H47a) in the solid-state structure of $[\mathrm{Pt}((\mathrm{R}, \mathrm{R})-\mathrm{Me}-$ Duphos $\left.)(\mathrm{Ph})\left(\mathrm{PMeIs}\left(\mathrm{CH}_{2} \mathrm{Ph}\right)\right)\right]\left[\mathrm{BF}_{4}\right](\mathbf{8})$. $\mathrm{H}$ atoms were placed in calculated positions.

\begin{tabular}{|l|l|l|}
\hline $\begin{array}{l}\text { Pt-Ph } \\
\text { Hydrogens }\end{array}$ & $\begin{array}{l}\text { d(H-H) to C41 } \\
\text { (P-Me) (ave) }\end{array}$ & $\begin{array}{l}\text { ((H-H) to C34 } \\
\left(\mathbf{P}-\mathbf{C H}_{2}\right) \\
(\text { ave })\end{array}$ \\
\hline H43a $(o)$ & 5.024 & 5.461 \\
\hline H44a $(m)$ & 6.156 & 7.411 \\
\hline H45a $(p)$ & 6.140 & 8.367 \\
\hline H46a $(m)$ & 4.933 & 7.707 \\
\hline H47a $(o)$ & 3.434 & 5.851 \\
\hline
\end{tabular}

The comparisons in Tables S5 and S6 are consistent with the idea that the major diastereomer of complex 4 in solution contains a $R_{P}$-PMeIs group, as observed in the solid state. These data suggest that other $\mathrm{P}-\mathrm{Me}$ positions (as modeled by the $\mathrm{P}-\mathrm{H}$ or $\mathrm{P}-\mathrm{CH}_{2} \mathrm{Ph}$ groups) would lead to 
longer distances between the $\mathrm{P}-\mathrm{Me}$ hydrogens and the $\mathrm{Pt}-\mathrm{Ph}$ ring, which are potentially inconsistent with the observed P-Me/Pt-Ph NOEs.

Summary. We conclude that the solution structure of the major diastereomer of $\mathrm{Pt}((\mathrm{R}, \mathrm{R})-\mathrm{Me}-$ Duphos $)(\mathrm{Ph})(\mathrm{PMeIs})$ is consistent with the solid-state structure of the Pt complex, which contains an $R_{P}$-Pt-PMeIs group. Note that we could not rule out the possibility that the $S_{P}$ diastereomer would also yield spectra consistent with these observations; the low concentration of this minor isomers precluded analogous NMR studies. However, indirect evidence from the structures of analogous Pt complexes is also consistent with the $R_{P}$ absolute configuration. ${ }^{2}$ 
Table 1. Crystal data and structure refinement for $\mathrm{Pt}((\mathrm{R}, \mathrm{R})-\mathrm{Me}-\mathrm{Duphos})(\mathrm{Ph})(\mathrm{OH})$, glu236.

Identification code

Empirical formula

Formula weight

Temperature

Wavelength

Crystal system

Space group

Unit cell dimensions

Volume

Z

Density (calculated)

Absorption coefficient

$\mathrm{F}(000)$

Crystal size

Theta range for data collection

Index ranges

Reflections collected

Independent reflections

Completeness to theta $=25.00^{\circ}$

Absorption correction

Max. and min. transmission

Refinement method

Data / restraints / parameters

Goodness-of-fit on $\mathrm{F}^{2}$

Final R indices [I $>2 \operatorname{sigma}(\mathrm{I})]$

$\mathrm{R}$ indices (all data)

Absolute structure parameter

Largest diff. peak and hole glu236

C24 H34 O P2 Pt

595.54

208(2) K

$0.71073 \AA$

Monoclinic

P2(1)

$\begin{array}{ll}\mathrm{a}=8.434(2) \AA & \alpha=90^{\circ} \\ \mathrm{b}=13.588(4) \AA & \beta=101.266(6)^{\circ} \\ \mathrm{c}=20.825(6) \AA & \gamma=90^{\circ}\end{array}$

2340.7(11) $\AA^{3}$

4

$1.690 \mathrm{~g} / \mathrm{cm}^{3}$

$6.143 \mathrm{~mm}^{-1}$

1176

$0.30 \times 0.20 \times 0.10 \mathrm{~mm}^{3}$

1.80 to $28.26^{\circ}$

$-10<=\mathrm{h}<=4,-18<=\mathrm{k}<=17,-22<=\mathrm{l}<=26$

12300

$9600[\mathrm{R}(\mathrm{int})=0.0304]$

$97.8 \%$

multi-scan

0.5786 and 0.2601

Full-matrix least-squares on $\mathrm{F}^{2}$

$9600 / 1 / 505$

1.003

$\mathrm{R} 1=0.0448, \mathrm{wR} 2=0.1002$

$\mathrm{R} 1=0.0481, \mathrm{wR} 2=0.1018$

$0.005(8)$

2.981 and -2.771 e $\AA^{-3}$ 
Table 2. Atomic coordinates $\left(\mathrm{x} 10^{4}\right)$ and equivalent isotropic displacement parameters $\left(\AA^{2} \times 10^{3}\right)$ for $\mathrm{Pt}((\mathrm{R}, \mathrm{R})-\mathrm{Me}-$ Duphos $)(\mathrm{Ph})(\mathrm{OH})$, glu236. $\mathrm{U}(\mathrm{eq})$ is defined as one third of the trace of the orthogonalized $\mathrm{U}^{\mathrm{ij}}$ tensor.

\begin{tabular}{|c|c|c|c|c|}
\hline & $\mathrm{x}$ & $\mathrm{y}$ & $\mathrm{z}$ & $\mathrm{U}(\mathrm{eq})$ \\
\hline $\operatorname{Pt}(1)$ & $4320(1)$ & 7386(1) & $6186(1)$ & $27(1)$ \\
\hline $\operatorname{Pt}\left(1^{\prime}\right)$ & $5744(1)$ & $2622(1)$ & $8730(1)$ & $32(1)$ \\
\hline $\mathrm{P}\left(1^{\prime}\right)$ & $8234(3)$ & $3057(2)$ & 9144(1) & $31(1)$ \\
\hline $\mathrm{P}(1)$ & 2065(3) & $8181(2)$ & 5792(1) & $30(1)$ \\
\hline $\mathrm{P}\left(2^{\prime}\right)$ & $5869(3)$ & $3741(2)$ & 7917(1) & $37(1)$ \\
\hline $\mathrm{P}(2)$ & $4580(3)$ & $8405(2)$ & 7072(1) & $30(1)$ \\
\hline $\mathrm{O}\left(1^{\prime}\right)$ & $3428(8)$ & 2206(6) & $8322(3)$ & $60(2)$ \\
\hline $\mathrm{O}(1)$ & 6351(9) & 6601(5) & 6599(3) & $49(2)$ \\
\hline$C\left(1^{\prime}\right)$ & $9909(12)$ & $2227(7)$ & $8996(5)$ & $44(2)$ \\
\hline $\mathrm{C}(1)$ & $163(10)$ & 7511(7) & $5789(4)$ & $40(2)$ \\
\hline $\mathrm{C}\left(2^{\prime}\right)$ & $10629(13)$ & $1840(8)$ & 9692(6) & $60(3)$ \\
\hline $\mathrm{C}(2)$ & $-447(12)$ & $7270(10)$ & $5057(5)$ & $57(3)$ \\
\hline$C\left(3^{\prime}\right)$ & $10625(13)$ & $2675(9)$ & $10178(5)$ & $60(3)$ \\
\hline $\mathrm{C}(3)$ & $-186(13)$ & $8183(9)$ & 4673(5) & $60(3)$ \\
\hline$C\left(4^{\prime}\right)$ & $8901(12)$ & $3104(7)$ & $10042(4)$ & $42(2)$ \\
\hline $\mathrm{C}(4)$ & $1566(11)$ & $8495(7)$ & 4909(4) & $38(2)$ \\
\hline$C\left(5^{\prime}\right)$ & $8639(11)$ & $4242(6)$ & 8773(4) & $31(2)$ \\
\hline$C(5)$ & 1953(10) & $9283(6)$ & $6300(4)$ & $29(2)$ \\
\hline $\mathrm{C}\left(6^{\prime}\right)$ & $10013(11)$ & $4804(6)$ & $9024(5)$ & $39(2)$ \\
\hline$C(6)$ & 706(11) & 9980(7) & 6123(5) & $39(2)$ \\
\hline$C\left(7^{\prime}\right)$ & $10238(12)$ & 5701(7) & $8732(5)$ & $48(3)$ \\
\hline$C(7)$ & $677(13)$ & $10800(7)$ & $6519(5)$ & $46(3)$ \\
\hline $\mathrm{C}\left(8^{\prime}\right)$ & $9179(14)$ & $6009(7)$ & $8189(5)$ & $49(3)$ \\
\hline $\mathrm{C}(8)$ & $1871(12)$ & 10947(6) & 7064(4) & $37(2)$ \\
\hline $\mathrm{C}\left(9^{\prime}\right)$ & $7825(13)$ & $5469(7)$ & 7937(5) & $45(2)$ \\
\hline $\mathrm{C}(9)$ & $3084(12)$ & $10269(7)$ & $7240(5)$ & $41(2)$ \\
\hline
\end{tabular}




\begin{tabular}{|c|c|c|c|c|}
\hline$C\left(10^{\prime}\right)$ & $7530(11)$ & $4561(6)$ & $8235(4)$ & $34(2)$ \\
\hline $\mathrm{C}(10)$ & $3128(10)$ & $9404(6)$ & $6858(4)$ & $30(2)$ \\
\hline $\mathrm{C}\left(11^{\prime}\right)$ & $4126(13)$ & $4482(8)$ & $7525(5)$ & $53(3)$ \\
\hline $\mathrm{C}(11)$ & $6530(11)$ & $8966(6)$ & $7429(4)$ & $36(2)$ \\
\hline$C\left(12^{\prime}\right)$ & $3570(15)$ & $3992(10)$ & $6856(5)$ & $64(4)$ \\
\hline $\mathrm{C}(12)$ & $6943(13)$ & $8611(8)$ & $8147(4)$ & $46(3)$ \\
\hline$C\left(13^{\prime}\right)$ & $5100(17)$ & $3776(9)$ & $6597(5)$ & $72(4)$ \\
\hline$C(13)$ & $5379(13)$ & $8474(7)$ & $8385(4)$ & $47(3)$ \\
\hline$C\left(14^{\prime}\right)$ & $6270(13)$ & $3246(8)$ & $7128(4)$ & $49(3)$ \\
\hline $\mathrm{C}(14)$ & $4229(12)$ & 7887(7) & 7854(4) & $42(2)$ \\
\hline$C\left(15^{\prime}\right)$ & $8730(14)$ & 4084(8) & 10389(5) & $53(3)$ \\
\hline$C(15)$ & 1959(13) & $9550(7)$ & $4703(5)$ & $51(3)$ \\
\hline$C\left(16^{\prime}\right)$ & $9448(14)$ & $1436(7)$ & $8501(6)$ & $57(3)$ \\
\hline$C(16)$ & $307(14)$ & $6616(8)$ & $6244(5)$ & $57(3)$ \\
\hline $\mathrm{C}\left(17^{\prime}\right)$ & $2807(16)$ & 4571(13) & $7895(6)$ & $106(6)$ \\
\hline $\mathrm{C}(17)$ & 7891(12) & $8777(8)$ & $7068(5)$ & $54(3)$ \\
\hline $\mathrm{C}\left(18^{\prime}\right)$ & $8035(16)$ & $3274(10)$ & $7039(6)$ & $80(4)$ \\
\hline $\mathrm{C}(18)$ & $2522(16)$ & $7870(9)$ & $7957(5)$ & $69(4)$ \\
\hline $\mathrm{C}\left(19^{\prime}\right)$ & $5642(11)$ & $1630(7)$ & $9467(5)$ & $36(2)$ \\
\hline $\mathrm{C}(19)$ & $4185(11)$ & $6550(6)$ & $5356(4)$ & $32(2)$ \\
\hline $\mathrm{C}\left(20^{\prime}\right)$ & $6022(13)$ & $628(7)$ & $9407(6)$ & $51(3)$ \\
\hline $\mathrm{C}(20)$ & $3551(12)$ & $5599(7)$ & $5264(5)$ & $40(2)$ \\
\hline $\mathrm{C}\left(21^{\prime}\right)$ & $5852(16)$ & $-64(8)$ & $9877(6)$ & $65(3)$ \\
\hline$C(21)$ & $3590(13)$ & $5039(8)$ & $4708(6)$ & $55(3)$ \\
\hline$C\left(22^{\prime}\right)$ & $5227(14)$ & 198(8) & $10420(5)$ & $54(3)$ \\
\hline $\mathrm{C}(22)$ & $4322(14)$ & $5417(10)$ & $4228(6)$ & $64(4)$ \\
\hline $\mathrm{C}\left(23^{\prime}\right)$ & $4883(15)$ & 1191(8) & $10502(5)$ & $54(3)$ \\
\hline$C(23)$ & $5014(14)$ & $6348(9)$ & $4303(5)$ & $51(3)$ \\
\hline $\mathrm{C}\left(24^{\prime}\right)$ & $5138(13)$ & 1883(7) & 10033(5) & $48(3)$ \\
\hline$C(24)$ & 4915(13) & $6889(7)$ & $4854(5)$ & $45(2)$ \\
\hline
\end{tabular}


Table 3. Bond lengths $[\AA ̊]$ and angles [deg] for $\mathrm{Pt}((\mathrm{R}, \mathrm{R})-\mathrm{Me}-\mathrm{Duphos})(\mathrm{Ph})(\mathrm{OH})$, glu236.

\begin{tabular}{|c|c|c|c|}
\hline $\mathrm{Pt}(1)-\mathrm{O}(1)$ & $2.058(6)$ & $C(4)-C(15)$ & $1.551(13)$ \\
\hline $\operatorname{Pt}(1)-C(19)$ & $2.053(9)$ & $\mathrm{C}\left(5^{\prime}\right)-\mathrm{C}\left(10^{\prime}\right)$ & $1.381(12)$ \\
\hline $\mathrm{Pt}(1)-\mathrm{P}(1)$ & $2.202(2)$ & $\mathrm{C}\left(5^{\prime}\right)-\mathrm{C}\left(6^{\prime}\right)$ & $1.401(12)$ \\
\hline $\mathrm{Pt}(1)-\mathrm{P}(2)$ & $2.282(2)$ & $C(5)-C(10)$ & $1.382(12)$ \\
\hline $\operatorname{Pt}\left(1^{\prime}\right)-\mathrm{O}\left(1^{\prime}\right)$ & $2.052(6)$ & $C(5)-C(6)$ & $1.410(12)$ \\
\hline $\operatorname{Pt}\left(1^{\prime}\right)-\mathrm{C}\left(19^{\prime}\right)$ & $2.056(10)$ & $\mathrm{C}\left(6^{\prime}\right)-\mathrm{C}\left(7^{\prime}\right)$ & $1.391(13)$ \\
\hline $\operatorname{Pt}\left(1^{\prime}\right)-\mathrm{P}\left(1^{\prime}\right)$ & $2.189(2)$ & $C(6)-C(7)$ & $1.388(13)$ \\
\hline $\operatorname{Pt}\left(1^{\prime}\right)-\mathrm{P}\left(2^{\prime}\right)$ & $2.295(3)$ & $\mathrm{C}\left(7^{\prime}\right)-\mathrm{C}\left(8^{\prime}\right)$ & $1.362(14)$ \\
\hline $\mathrm{P}\left(1^{\prime}\right)-\mathrm{C}\left(5^{\prime}\right)$ & $1.846(8)$ & $\mathrm{C}(7)-\mathrm{C}(8)$ & $1.377(13)$ \\
\hline $\mathrm{P}\left(1^{\prime}\right)-\mathrm{C}\left(4^{\prime}\right)$ & $1.847(9)$ & $\mathrm{C}\left(8^{\prime}\right)-\mathrm{C}\left(9^{\prime}\right)$ & $1.373(14)$ \\
\hline$P\left(1^{\prime}\right)-C\left(1^{\prime}\right)$ & $1.878(10)$ & $\mathrm{C}(8)-\mathrm{C}(9)$ & $1.372(13)$ \\
\hline $\mathrm{P}(1)-\mathrm{C}(1)$ & $1.843(9)$ & $\mathrm{C}\left(9^{\prime}\right)-\mathrm{C}\left(10^{\prime}\right)$ & $1.425(12)$ \\
\hline$P(1)-C(5)$ & $1.846(8)$ & $C(9)-C(10)$ & $1.424(11)$ \\
\hline $\mathrm{P}(1)-\mathrm{C}(4)$ & $1.855(9)$ & $\mathrm{C}\left(11^{\prime}\right)-\mathrm{C}\left(17^{\prime}\right)$ & $1.478(16)$ \\
\hline $\mathrm{P}\left(2^{\prime}\right)-\mathrm{C}\left(10^{\prime}\right)$ & $1.812(10)$ & $\mathrm{C}\left(11^{\prime}\right)-\mathrm{C}\left(12^{\prime}\right)$ & $1.533(14)$ \\
\hline $\mathrm{P}\left(2^{\prime}\right)-\mathrm{C}\left(11^{\prime}\right)$ & $1.836(10)$ & $\mathrm{C}(11)-\mathrm{C}(17)$ & $1.512(13)$ \\
\hline $\mathrm{P}\left(2^{\prime}\right)-\mathrm{C}\left(14^{\prime}\right)$ & $1.867(9)$ & $\mathrm{C}(11)-\mathrm{C}(12)$ & $1.543(12)$ \\
\hline$P(2)-C(10)$ & $1.824(8)$ & $\mathrm{C}\left(12^{\prime}\right)-\mathrm{C}\left(13^{\prime}\right)$ & $1.521(18)$ \\
\hline$P(2)-C(11)$ & $1.834(9)$ & $\mathrm{C}(12)-\mathrm{C}(13)$ & $1.509(15)$ \\
\hline$P(2)-C(14)$ & $1.851(9)$ & $\mathrm{C}\left(13^{\prime}\right)-\mathrm{C}\left(14^{\prime}\right)$ & $1.513(14)$ \\
\hline$C\left(1^{\prime}\right)-C\left(16^{\prime}\right)$ & $1.488(14)$ & $\mathrm{C}(13)-\mathrm{C}(14)$ & $1.543(13)$ \\
\hline $\mathrm{C}\left(1^{\prime}\right)-\mathrm{C}\left(2^{\prime}\right)$ & $1.550(14)$ & $\mathrm{C}\left(14^{\prime}\right)-\mathrm{C}\left(18^{\prime}\right)$ & $1.536(16)$ \\
\hline$C(1)-C(16)$ & $1.532(14)$ & $\mathrm{C}(14)-\mathrm{C}(18)$ & $1.497(15)$ \\
\hline$C(1)-C(2)$ & $1.547(13)$ & $\mathrm{C}\left(19^{\prime}\right)-\mathrm{C}\left(24^{\prime}\right)$ & $1.373(13)$ \\
\hline$C\left(2^{\prime}\right)-C\left(3^{\prime}\right)$ & $1.521(15)$ & $\mathrm{C}\left(19^{\prime}\right)-\mathrm{C}\left(20^{\prime}\right)$ & $1.411(13)$ \\
\hline$C(2)-C(3)$ & $1.514(16)$ & $\mathrm{C}(19)-\mathrm{C}(24)$ & $1.393(13)$ \\
\hline $\mathrm{C}\left(3^{\prime}\right)-\mathrm{C}\left(4^{\prime}\right)$ & $1.541(14)$ & $\mathrm{C}(19)-\mathrm{C}(20)$ & $1.397(12)$ \\
\hline$C(3)-C(4)$ & $1.524(14)$ & $\mathrm{C}\left(20^{\prime}\right)-\mathrm{C}\left(21^{\prime}\right)$ & $1.383(15)$ \\
\hline$C\left(4^{\prime}\right)-C\left(15^{\prime}\right)$ & $1.535(14)$ & $C(20)-C(21)$ & $1.391(13)$ \\
\hline
\end{tabular}




\begin{tabular}{|c|c|c|c|}
\hline $\mathrm{C}\left(21^{\prime}\right)-\mathrm{C}\left(22^{\prime}\right)$ & $1.385(15)$ & $\mathrm{C}\left(10^{\prime}\right)-\mathrm{P}\left(2^{\prime}\right)-\mathrm{C}\left(11^{\prime}\right)$ & $108.6(5)$ \\
\hline$C(21)-C(22)$ & $1.374(16)$ & $\mathrm{C}\left(10^{\prime}\right)-\mathrm{P}\left(2^{\prime}\right)-\mathrm{C}\left(14^{\prime}\right)$ & $106.5(5)$ \\
\hline $\mathrm{C}\left(22^{\prime}\right)-\mathrm{C}\left(23^{\prime}\right)$ & $1.397(15)$ & $\mathrm{C}\left(11^{\prime}\right)-\mathrm{P}\left(2^{\prime}\right)-\mathrm{C}\left(14^{\prime}\right)$ & $94.2(5)$ \\
\hline$C(22)-C(23)$ & $1.389(16)$ & $\mathrm{C}\left(10^{\prime}\right)-\mathrm{P}\left(2^{\prime}\right)-\mathrm{Pt}\left(1^{\prime}\right)$ & $106.3(3)$ \\
\hline $\mathrm{C}\left(23^{\prime}\right)-\mathrm{C}\left(24^{\prime}\right)$ & $1.402(14)$ & $\mathrm{C}\left(11^{\prime}\right)-\mathrm{P}\left(2^{\prime}\right)-\mathrm{Pt}\left(1^{\prime}\right)$ & $122.9(4)$ \\
\hline \multirow[t]{2}{*}{$C(23)-C(24)$} & $1.378(13)$ & $\mathrm{C}\left(14^{\prime}\right)-\mathrm{P}\left(2^{\prime}\right)-\mathrm{Pt}\left(1^{\prime}\right)$ & $117.0(3)$ \\
\hline & & $\mathrm{C}(10)-\mathrm{P}(2)-\mathrm{C}(11)$ & 107.2(4) \\
\hline $\mathrm{O}(1)-\mathrm{Pt}(1)-\mathrm{C}(19)$ & $88.3(3)$ & $\mathrm{C}(10)-\mathrm{P}(2)-\mathrm{C}(14)$ & $106.4(4)$ \\
\hline $\mathrm{O}(1)-\mathrm{Pt}(1)-\mathrm{P}(1)$ & $176.2(2)$ & $\mathrm{C}(11)-\mathrm{P}(2)-\mathrm{C}(14)$ & $94.9(4)$ \\
\hline $\mathrm{C}(19)-\mathrm{Pt}(1)-\mathrm{P}(1)$ & $93.2(3)$ & $\mathrm{C}(10)-\mathrm{P}(2)-\mathrm{Pt}(1)$ & $107.5(3)$ \\
\hline $\mathrm{O}(1)-\mathrm{Pt}(1)-\mathrm{P}(2)$ & $92.07(19)$ & $\mathrm{C}(11)-\mathrm{P}(2)-\mathrm{Pt}(1)$ & $121.2(3)$ \\
\hline $\mathrm{C}(19)-\mathrm{Pt}(1)-\mathrm{P}(2)$ & $175.5(3)$ & $\mathrm{C}(14)-\mathrm{P}(2)-\mathrm{Pt}(1)$ & $118.2(3)$ \\
\hline $\mathrm{P}(1)-\mathrm{Pt}(1)-\mathrm{P}(2)$ & $86.74(8)$ & $\mathrm{C}\left(16^{\prime}\right)-\mathrm{C}\left(1^{\prime}\right)-\mathrm{C}\left(2^{\prime}\right)$ & $113.9(8)$ \\
\hline $\mathrm{O}\left(1^{\prime}\right)-\operatorname{Pt}\left(1^{\prime}\right)-\mathrm{C}\left(19^{\prime}\right)$ & $87.3(3)$ & $\mathrm{C}\left(16^{\prime}\right)-\mathrm{C}\left(1^{\prime}\right)-\mathrm{P}\left(1^{\prime}\right)$ & $116.3(7)$ \\
\hline $\mathrm{O}\left(1^{\prime}\right)-\operatorname{Pt}\left(1^{\prime}\right)-\mathrm{P}\left(1^{\prime}\right)$ & $178.6(2)$ & $\mathrm{C}\left(2^{\prime}\right)-\mathrm{C}\left(1^{\prime}\right)-\mathrm{P}\left(1^{\prime}\right)$ & $102.8(7)$ \\
\hline$C\left(19^{\prime}\right)-\operatorname{Pt}\left(1^{\prime}\right)-P\left(1^{\prime}\right)$ & $93.5(2)$ & $\mathrm{C}(16)-\mathrm{C}(1)-\mathrm{C}(2)$ & $114.3(9)$ \\
\hline $\mathrm{O}\left(1^{\prime}\right)-\operatorname{Pt}\left(1^{\prime}\right)-\mathrm{P}\left(2^{\prime}\right)$ & $93.1(2)$ & $\mathrm{C}(16)-\mathrm{C}(1)-\mathrm{P}(1)$ & $115.3(7)$ \\
\hline$C\left(19^{\prime}\right)-\operatorname{Pt}\left(1^{\prime}\right)-P\left(2^{\prime}\right)$ & $179.4(3)$ & $\mathrm{C}(2)-\mathrm{C}(1)-\mathrm{P}(1)$ & $103.3(6)$ \\
\hline $\mathrm{P}\left(1^{\prime}\right)-\mathrm{Pt}\left(1^{\prime}\right)-\mathrm{P}\left(2^{\prime}\right)$ & $86.14(9)$ & $\mathrm{C}\left(3^{\prime}\right)-\mathrm{C}\left(2^{\prime}\right)-\mathrm{C}\left(1^{\prime}\right)$ & $108.5(8)$ \\
\hline $\mathrm{C}\left(5^{\prime}\right)-\mathrm{P}\left(1^{\prime}\right)-\mathrm{C}\left(4^{\prime}\right)$ & $110.4(4)$ & $\mathrm{C}(3)-\mathrm{C}(2)-\mathrm{C}(1)$ & $107.0(9)$ \\
\hline $\mathrm{C}\left(5^{\prime}\right)-\mathrm{P}\left(1^{\prime}\right)-\mathrm{C}\left(1^{\prime}\right)$ & $104.4(4)$ & $\mathrm{C}\left(2^{\prime}\right)-\mathrm{C}\left(3^{\prime}\right)-\mathrm{C}\left(4^{\prime}\right)$ & $106.5(8)$ \\
\hline $\mathrm{C}\left(4^{\prime}\right)-\mathrm{P}\left(1^{\prime}\right)-\mathrm{C}\left(1^{\prime}\right)$ & $95.5(4)$ & $\mathrm{C}(2)-\mathrm{C}(3)-\mathrm{C}(4)$ & $106.9(8)$ \\
\hline $\mathrm{C}\left(5^{\prime}\right)-\mathrm{P}\left(1^{\prime}\right)-\mathrm{Pt}\left(1^{\prime}\right)$ & 108.2(3) & $\mathrm{C}\left(3^{\prime}\right)-\mathrm{C}\left(4^{\prime}\right)-\mathrm{C}\left(15^{\prime}\right)$ & $114.4(8)$ \\
\hline $\mathrm{C}\left(4^{\prime}\right)-\mathrm{P}\left(1^{\prime}\right)-\operatorname{Pt}\left(1^{\prime}\right)$ & 119.1(3) & $\mathrm{C}\left(3^{\prime}\right)-\mathrm{C}\left(4^{\prime}\right)-\mathrm{P}\left(1^{\prime}\right)$ & $105.3(7)$ \\
\hline $\mathrm{C}\left(1^{\prime}\right)-\mathrm{P}\left(1^{\prime}\right)-\operatorname{Pt}\left(1^{\prime}\right)$ & $117.9(3)$ & $\mathrm{C}\left(15^{\prime}\right)-\mathrm{C}\left(4^{\prime}\right)-\mathrm{P}\left(1^{\prime}\right)$ & $118.0(7)$ \\
\hline $\mathrm{C}(1)-\mathrm{P}(1)-\mathrm{C}(5)$ & $105.1(4)$ & $\mathrm{C}(3)-\mathrm{C}(4)-\mathrm{C}(15)$ & $114.5(8)$ \\
\hline $\mathrm{C}(1)-\mathrm{P}(1)-\mathrm{C}(4)$ & $94.7(4)$ & $\mathrm{C}(3)-\mathrm{C}(4)-\mathrm{P}(1)$ & $105.8(7)$ \\
\hline $\mathrm{C}(5)-\mathrm{P}(1)-\mathrm{C}(4)$ & $110.8(4)$ & $\mathrm{C}(15)-\mathrm{C}(4)-\mathrm{P}(1)$ & $117.8(6)$ \\
\hline $\mathrm{C}(1)-\mathrm{P}(1)-\mathrm{Pt}(1)$ & $116.5(3)$ & $\mathrm{C}\left(10^{\prime}\right)-\mathrm{C}\left(5^{\prime}\right)-\mathrm{C}\left(6^{\prime}\right)$ & $120.7(8)$ \\
\hline $\mathrm{C}(5)-\mathrm{P}(1)-\mathrm{Pt}(1)$ & $108.7(3)$ & $\mathrm{C}\left(10^{\prime}\right)-\mathrm{C}\left(5^{\prime}\right)-\mathrm{P}\left(1^{\prime}\right)$ & $117.5(6)$ \\
\hline $\mathrm{C}(4)-\mathrm{P}(1)-\mathrm{Pt}(1)$ & $119.6(3)$ & $\mathrm{C}\left(6^{\prime}\right)-\mathrm{C}\left(5^{\prime}\right)-\mathrm{P}\left(1^{\prime}\right)$ & $121.8(7)$ \\
\hline
\end{tabular}




\begin{tabular}{|c|c|c|c|}
\hline$C(10)-C(5)-C(6)$ & $121.0(8)$ & $\mathrm{C}(18)-\mathrm{C}(14)-\mathrm{P}(2)$ & $117.1(7)$ \\
\hline$C(10)-C(5)-P(1)$ & $118.1(6)$ & $\mathrm{C}(13)-\mathrm{C}(14)-\mathrm{P}(2)$ & $104.3(6)$ \\
\hline $\mathrm{C}(6)-\mathrm{C}(5)-\mathrm{P}(1)$ & $120.9(7)$ & $\mathrm{C}\left(24^{\prime}\right)-\mathrm{C}\left(19^{\prime}\right)-\mathrm{C}\left(20^{\prime}\right)$ & $115.5(9)$ \\
\hline $\mathrm{C}\left(7^{\prime}\right)-\mathrm{C}\left(6^{\prime}\right)-\mathrm{C}\left(5^{\prime}\right)$ & 119.2(9) & $\mathrm{C}\left(24^{\prime}\right)-\mathrm{C}\left(19^{\prime}\right)-\mathrm{Pt}\left(1^{\prime}\right)$ & $122.8(7)$ \\
\hline$C(7)-C(6)-C(5)$ & $118.9(9)$ & $\mathrm{C}\left(20^{\prime}\right)-\mathrm{C}\left(19^{\prime}\right)-\mathrm{Pt}\left(1^{\prime}\right)$ & $121.6(8)$ \\
\hline $\mathrm{C}\left(8^{\prime}\right)-\mathrm{C}\left(7^{\prime}\right)-\mathrm{C}\left(6^{\prime}\right)$ & $120.5(10)$ & $\mathrm{C}(24)-\mathrm{C}(19)-\mathrm{C}(20)$ & $114.5(9)$ \\
\hline$C(6)-C(7)-C(8)$ & $120.7(9)$ & $\mathrm{C}(24)-\mathrm{C}(19)-\mathrm{Pt}(1)$ & $119.4(7)$ \\
\hline $\mathrm{C}\left(7^{\prime}\right)-\mathrm{C}\left(8^{\prime}\right)-\mathrm{C}\left(9^{\prime}\right)$ & $121.0(9)$ & $\mathrm{C}(20)-\mathrm{C}(19)-\mathrm{Pt}(1)$ & $125.8(7)$ \\
\hline $\mathrm{C}(9)-\mathrm{C}(8)-\mathrm{C}(7)$ & $120.7(8)$ & $\mathrm{C}\left(21^{\prime}\right)-\mathrm{C}\left(20^{\prime}\right)-\mathrm{C}\left(19^{\prime}\right)$ & $122.5(10)$ \\
\hline $\mathrm{C}\left(8^{\prime}\right)-\mathrm{C}\left(9^{\prime}\right)-\mathrm{C}\left(10^{\prime}\right)$ & $119.9(9)$ & $\mathrm{C}(21)-\mathrm{C}(20)-\mathrm{C}(19)$ & $123.4(10)$ \\
\hline $\mathrm{C}(8)-\mathrm{C}(9)-\mathrm{C}(10)$ & $120.2(9)$ & $\mathrm{C}\left(22^{\prime}\right)-\mathrm{C}\left(21^{\prime}\right)-\mathrm{C}\left(20^{\prime}\right)$ & $120.7(10)$ \\
\hline $\mathrm{C}\left(5^{\prime}\right)-\mathrm{C}\left(10^{\prime}\right)-\mathrm{C}\left(9^{\prime}\right)$ & $118.5(9)$ & $\mathrm{C}(22)-\mathrm{C}(21)-\mathrm{C}(20)$ & $119.3(10)$ \\
\hline $\mathrm{C}\left(5^{\prime}\right)-\mathrm{C}\left(10^{\prime}\right)-\mathrm{P}\left(2^{\prime}\right)$ & $117.1(6)$ & $\mathrm{C}\left(21^{\prime}\right)-\mathrm{C}\left(22^{\prime}\right)-\mathrm{C}\left(23^{\prime}\right)$ & 118.0(10) \\
\hline $\mathrm{C}\left(9^{\prime}\right)-\mathrm{C}\left(10^{\prime}\right)-\mathrm{P}\left(2^{\prime}\right)$ & $124.1(7)$ & $\mathrm{C}(21)-\mathrm{C}(22)-\mathrm{C}(23)$ & 119.7(10) \\
\hline $\mathrm{C}(5)-\mathrm{C}(10)-\mathrm{C}(9)$ & $118.4(8)$ & $\mathrm{C}\left(24^{\prime}\right)-\mathrm{C}\left(23^{\prime}\right)-\mathrm{C}\left(22^{\prime}\right)$ & 119.9(11) \\
\hline$C(5)-C(10)-P(2)$ & $116.9(6)$ & $\mathrm{C}(22)-\mathrm{C}(23)-\mathrm{C}(24)$ & 119.2(10) \\
\hline $\mathrm{C}(9)-\mathrm{C}(10)-\mathrm{P}(2)$ & $124.6(7)$ & $\mathrm{C}\left(19^{\prime}\right)-\mathrm{C}\left(24^{\prime}\right)-\mathrm{C}\left(23^{\prime}\right)$ & 123.1(10) \\
\hline $\mathrm{C}\left(17^{\prime}\right)-\mathrm{C}\left(11^{\prime}\right)-\mathrm{C}\left(12^{\prime}\right)$ & $112.5(10)$ & $\mathrm{C}(23)-\mathrm{C}(24)-\mathrm{C}(19)$ & $123.8(10)$ \\
\hline $\mathrm{C}\left(17^{\prime}\right)-\mathrm{C}\left(11^{\prime}\right)-\mathrm{P}\left(2^{\prime}\right)$ & $115.6(8)$ & & \\
\hline $\mathrm{C}\left(12^{\prime}\right)-\mathrm{C}\left(11^{\prime}\right)-\mathrm{P}\left(2^{\prime}\right)$ & $104.0(8)$ & & \\
\hline $\mathrm{C}(17)-\mathrm{C}(11)-\mathrm{C}(12)$ & $112.3(8)$ & \multirow{2}{*}{\multicolumn{2}{|c|}{$\begin{array}{l}\text { Symmetry transformations used to generate equivalent } \\
\text { atoms: }\end{array}$}} \\
\hline $\mathrm{C}(17)-\mathrm{C}(11)-\mathrm{P}(2)$ & $115.8(7)$ & & \\
\hline $\mathrm{C}(12)-\mathrm{C}(11)-\mathrm{P}(2)$ & $106.1(6)$ & & \\
\hline $\mathrm{C}\left(13^{\prime}\right)-\mathrm{C}\left(12^{\prime}\right)-\mathrm{C}\left(11^{\prime}\right)$ & $106.0(9)$ & & \\
\hline $\mathrm{C}(13)-\mathrm{C}(12)-\mathrm{C}(11)$ & $108.1(8)$ & & \\
\hline $\mathrm{C}\left(14^{\prime}\right)-\mathrm{C}\left(13^{\prime}\right)-\mathrm{C}\left(12^{\prime}\right)$ & $108.1(9)$ & & \\
\hline$C(12)-C(13)-C(14)$ & $107.2(8)$ & & \\
\hline $\mathrm{C}\left(13^{\prime}\right)-\mathrm{C}\left(14^{\prime}\right)-\mathrm{C}\left(18^{\prime}\right)$ & $114.2(10)$ & & \\
\hline $\mathrm{C}\left(13^{\prime}\right)-\mathrm{C}\left(14^{\prime}\right)-\mathrm{P}\left(2^{\prime}\right)$ & $105.5(7)$ & & \\
\hline $\mathrm{C}\left(18^{\prime}\right)-\mathrm{C}\left(14^{\prime}\right)-\mathrm{P}\left(2^{\prime}\right)$ & $116.3(8)$ & & \\
\hline$C(18)-C(14)-C(13)$ & 113.1(9) & & \\
\hline
\end{tabular}


Table 4. Anisotropic displacement parameters $\left(\AA^{2} \times 10^{3}\right)$ for $\operatorname{Pt}((\mathrm{R}, \mathrm{R})-\mathrm{Me}-\mathrm{Duphos})(\mathrm{Ph})(\mathrm{OH})$, glu236. The anisotropic displacement factor exponent takes the form: $-2 \pi^{2}\left[h^{2} a^{* 2} U^{11}+\ldots+2 h k a^{*} b^{*} U^{12}\right]$

\begin{tabular}{|c|c|c|c|c|c|c|}
\hline & $\mathrm{U}^{11}$ & $\mathrm{U}^{22}$ & $\mathrm{U}^{33}$ & $\mathrm{U}^{23}$ & $\mathrm{U}^{13}$ & $\mathrm{U}^{12}$ \\
\hline $\operatorname{Pt}(1)$ & $33(1)$ & $25(1)$ & $23(1)$ & $1(1)$ & $6(1)$ & $4(1)$ \\
\hline $\operatorname{Pt}\left(1^{\prime}\right)$ & $35(1)$ & $35(1)$ & $26(1)$ & $-7(1)$ & $7(1)$ & $-7(1)$ \\
\hline $\mathrm{P}\left(1^{\prime}\right)$ & $34(1)$ & $26(1)$ & $34(1)$ & $3(1)$ & $7(1)$ & $-1(1)$ \\
\hline $\mathrm{P}(1)$ & $34(1)$ & $27(1)$ & $28(1)$ & $-4(1)$ & $4(1)$ & $3(1)$ \\
\hline $\mathrm{P}\left(2^{\prime}\right)$ & $45(2)$ & $41(1)$ & $25(1)$ & $-4(1)$ & $3(1)$ & $6(1)$ \\
\hline $\mathrm{P}(2)$ & $39(1)$ & $28(1)$ & $23(1)$ & $1(1)$ & $5(1)$ & $-2(1)$ \\
\hline $\mathrm{O}\left(1^{\prime}\right)$ & $35(4)$ & $98(7)$ & $45(4)$ & $-13(4)$ & $5(3)$ & $-24(4)$ \\
\hline $\mathrm{O}(1)$ & $67(5)$ & $34(4)$ & $39(4)$ & $-7(3)$ & $-10(3)$ & $24(3)$ \\
\hline$C\left(1^{\prime}\right)$ & $43(5)$ & $28(6)$ & $62(6)$ & $12(5)$ & $11(5)$ & $-2(4)$ \\
\hline $\mathrm{C}(1)$ & $24(4)$ & $36(6)$ & $59(6)$ & $-17(5)$ & $3(4)$ & $-8(4)$ \\
\hline$C\left(2^{\prime}\right)$ & $51(7)$ & $37(6)$ & $88(9)$ & $15(6)$ & $3(6)$ & $4(5)$ \\
\hline $\mathrm{C}(2)$ & $28(5)$ & $60(8)$ & $79(8)$ & $-13(7)$ & $0(5)$ & $-7(5)$ \\
\hline$C\left(3^{\prime}\right)$ & $62(7)$ & $42(6)$ & $61(7)$ & $10(6)$ & $-22(5)$ & $-2(6)$ \\
\hline $\mathrm{C}(3)$ & $64(7)$ & $60(7)$ & $44(6)$ & $-16(5)$ & $-19(6)$ & $6(6)$ \\
\hline $\mathrm{C}\left(4^{\prime}\right)$ & $50(6)$ & $42(5)$ & $29(5)$ & $9(4)$ & $-4(4)$ & $-11(4)$ \\
\hline$C(4)$ & $36(5)$ & $44(5)$ & $31(5)$ & $-8(4)$ & $0(4)$ & $16(4)$ \\
\hline$C\left(5^{\prime}\right)$ & $37(5)$ & $25(4)$ & $32(5)$ & $-3(3)$ & $9(4)$ & $-8(3)$ \\
\hline$C(5)$ & $30(5)$ & $32(4)$ & $31(5)$ & 1(3) & $18(4)$ & $0(3)$ \\
\hline$C\left(6^{\prime}\right)$ & $37(5)$ & $32(5)$ & $49(6)$ & $9(4)$ & $9(5)$ & $-1(4)$ \\
\hline$C(6)$ & $45(6)$ & $35(5)$ & $37(5)$ & $3(4)$ & $10(4)$ & $4(4)$ \\
\hline$C\left(7^{\prime}\right)$ & $44(6)$ & $37(6)$ & $70(8)$ & $-1(5)$ & $26(5)$ & $-8(4)$ \\
\hline$C(7)$ & $66(7)$ & $33(5)$ & $42(6)$ & $-2(4)$ & $18(5)$ & $19(5)$ \\
\hline$C\left(8^{\prime}\right)$ & $70(8)$ & $22(5)$ & $63(7)$ & $12(4)$ & $33(6)$ & $12(5)$ \\
\hline $\mathrm{C}(8)$ & $54(6)$ & $22(4)$ & $41(5)$ & $-7(4)$ & $20(5)$ & $-1(4)$ \\
\hline $\mathrm{C}\left(9^{\prime}\right)$ & $58(7)$ & $37(5)$ & $44(6)$ & $12(4)$ & $19(5)$ & $12(5)$ \\
\hline $\mathrm{C}(9)$ & $53(6)$ & $36(5)$ & $33(5)$ & $-10(4)$ & $6(5)$ & $-8(4)$ \\
\hline
\end{tabular}




\begin{tabular}{|c|c|c|c|c|c|c|}
\hline $\mathrm{C}\left(10^{\prime}\right)$ & $36(5)$ & $37(5)$ & $29(5)$ & $5(4)$ & $9(4)$ & $10(4)$ \\
\hline $\mathrm{C}(10)$ & $40(5)$ & $26(4)$ & $26(4)$ & 1(3) & $13(4)$ & $2(3)$ \\
\hline $\mathrm{C}\left(11^{\prime}\right)$ & $55(7)$ & $53(7)$ & $43(6)$ & $-8(5)$ & $-8(5)$ & $9(5)$ \\
\hline $\mathrm{C}(11)$ & $44(6)$ & $23(4)$ & $41(5)$ & 1(4) & $8(4)$ & $3(4)$ \\
\hline$C\left(12^{\prime}\right)$ & $75(9)$ & $75(9)$ & $34(6)$ & $2(6)$ & $-8(6)$ & $-4(7)$ \\
\hline$C(12)$ & $54(7)$ & $47(6)$ & $34(5)$ & 2(4) & $-2(5)$ & $4(5)$ \\
\hline $\mathrm{C}\left(13^{\prime}\right)$ & $116(12)$ & $66(8)$ & $31(6)$ & $-10(5)$ & $12(7)$ & $-7(8)$ \\
\hline$C(13)$ & $82(8)$ & $39(5)$ & $20(5)$ & $5(4)$ & $6(5)$ & $6(5)$ \\
\hline$C\left(14^{\prime}\right)$ & $77(8)$ & $44(6)$ & $25(5)$ & $-4(4)$ & $11(5)$ & $0(5)$ \\
\hline$C(14)$ & $59(6)$ & $40(5)$ & $30(5)$ & $0(4)$ & $13(4)$ & $-7(5)$ \\
\hline$C\left(15^{\prime}\right)$ & $58(7)$ & $61(7)$ & $35(6)$ & $0(5)$ & $0(5)$ & $-1(6)$ \\
\hline$C(15)$ & $70(8)$ & $45(6)$ & $38(6)$ & $8(5)$ & $9(5)$ & $4(5)$ \\
\hline$C\left(16^{\prime}\right)$ & $73(8)$ & $32(5)$ & $80(8)$ & $-3(5)$ & $47(7)$ & $5(5)$ \\
\hline$C(16)$ & $55(7)$ & $56(7)$ & $65(8)$ & $-17(6)$ & $18(6)$ & $-23(6)$ \\
\hline$C\left(17^{\prime}\right)$ & $84(11)$ & $169(18)$ & $61(9)$ & $-9(10)$ & $2(8)$ & $74(11)$ \\
\hline $\mathrm{C}(17)$ & $42(6)$ & $58(7)$ & $61(7)$ & $0(6)$ & $3(5)$ & $-2(5)$ \\
\hline$C\left(18^{\prime}\right)$ & 101(11) & $80(10)$ & $72(9)$ & $-2(7)$ & $48(8)$ & $32(8)$ \\
\hline $\mathrm{C}(18)$ & $104(10)$ & $68(8)$ & $42(6)$ & $-3(6)$ & $28(6)$ & $-25(7)$ \\
\hline$C\left(19^{\prime}\right)$ & $35(5)$ & $33(5)$ & $41(5)$ & $-8(4)$ & $5(4)$ & $-16(4)$ \\
\hline $\mathrm{C}(19)$ & $44(5)$ & $27(4)$ & $22(4)$ & $-2(3)$ & $0(4)$ & $3(4)$ \\
\hline$C\left(20^{\prime}\right)$ & $59(7)$ & $37(5)$ & $68(7)$ & $-17(5)$ & $39(6)$ & $-9(5)$ \\
\hline $\mathrm{C}(20)$ & $44(6)$ & $32(5)$ & $46(6)$ & $-4(4)$ & $12(5)$ & $-2(4)$ \\
\hline $\mathrm{C}\left(21^{\prime}\right)$ & $89(9)$ & $23(5)$ & $91(10)$ & $0(5)$ & $36(8)$ & $-5(5)$ \\
\hline$C(21)$ & $58(7)$ & $42(6)$ & $66(8)$ & $-27(5)$ & $12(6)$ & $-1(5)$ \\
\hline $\mathrm{C}\left(22^{\prime}\right)$ & 71(8) & $44(6)$ & $39(6)$ & $12(5)$ & $-9(6)$ & $-18(5)$ \\
\hline $\mathrm{C}(22)$ & $57(8)$ & $87(10)$ & $48(7)$ & $-36(6)$ & $10(6)$ & $4(6)$ \\
\hline $\mathrm{C}\left(23^{\prime}\right)$ & $72(8)$ & $52(7)$ & $40(6)$ & $-1(5)$ & $16(6)$ & $-1(6)$ \\
\hline$C(23)$ & $64(8)$ & $61(7)$ & $31(6)$ & $-1(5)$ & $19(5)$ & $6(6)$ \\
\hline$C\left(24^{\prime}\right)$ & $69(7)$ & $32(5)$ & $42(6)$ & $-4(4)$ & $5(5)$ & $-1(5)$ \\
\hline $\mathrm{C}(24)$ & $65(7)$ & $30(5)$ & $40(6)$ & $-2(4)$ & $12(5)$ & $-1(5)$ \\
\hline
\end{tabular}


Table 5. Hydrogen coordinates (x $\left.10^{4}\right)$ and isotropic displacement parameters $\left(\AA^{2} \times 10^{3}\right)$ for $\mathrm{Pt}((\mathrm{R}, \mathrm{R})-\mathrm{Me}-$ Duphos)(Ph)(OH), glu236.

\begin{tabular}{|c|c|c|c|c|}
\hline & $\mathrm{x}$ & $\mathrm{y}$ & $\mathrm{z}$ & $\mathrm{U}(\mathrm{eq})$ \\
\hline $\mathrm{H}\left(1^{\prime} \mathrm{A}\right)$ & 3121 & 1775 & 8553 & 90 \\
\hline $\mathrm{H}(1 \mathrm{~A})$ & 6506 & 6156 & 6346 & 74 \\
\hline $\mathrm{H}\left(1^{\prime} \mathrm{B}\right)$ & 10738 & 2643 & 8852 & 53 \\
\hline $\mathrm{H}(1 \mathrm{~B})$ & -612 & 7976 & 5926 & 49 \\
\hline $\mathrm{H}\left(2^{\prime} \mathrm{A}\right)$ & 9981 & 1289 & 9801 & 72 \\
\hline $\mathrm{H}\left(2^{\prime} \mathrm{B}\right)$ & 11737 & 1607 & 9710 & 72 \\
\hline $\mathrm{H}(2 \mathrm{~A})$ & 157 & 6714 & 4926 & 69 \\
\hline $\mathrm{H}(2 \mathrm{~B})$ & -1597 & 7097 & 4977 & 69 \\
\hline $\mathrm{H}\left(3^{\prime} \mathrm{A}\right)$ & 11412 & 3181 & 10118 & 72 \\
\hline $\mathrm{H}\left(3^{\prime} \mathrm{B}\right)$ & 10906 & 2427 & 10628 & 72 \\
\hline $\mathrm{H}(3 \mathrm{~A})$ & -920 & 8709 & 4752 & 72 \\
\hline $\mathrm{H}(3 \mathrm{~B})$ & -391 & 8038 & 4203 & 72 \\
\hline $\mathrm{H}\left(4^{\prime} \mathrm{A}\right)$ & 8221 & 2624 & 10223 & 50 \\
\hline $\mathrm{H}(4 \mathrm{~A})$ & 2221 & 8047 & 4691 & 46 \\
\hline $\mathrm{H}\left(6^{\prime} \mathrm{A}\right)$ & 10775 & 4577 & 9385 & 47 \\
\hline $\mathrm{H}(6 \mathrm{~A})$ & -91 & 9892 & 5744 & 47 \\
\hline $\mathrm{H}\left(7^{\prime} \mathrm{A}\right)$ & 11127 & 6098 & 8912 & 58 \\
\hline $\mathrm{H}(7 \mathrm{~A})$ & -168 & 11259 & 6414 & 55 \\
\hline $\mathrm{H}\left(8^{\prime} \mathrm{A}\right)$ & 9379 & 6600 & 7985 & 58 \\
\hline $\mathrm{H}(8 \mathrm{~A})$ & 1856 & 11518 & 7317 & 45 \\
\hline $\mathrm{H}\left(9^{\prime} \mathrm{A}\right)$ & 7093 & 5698 & 7568 & 54 \\
\hline $\mathrm{H}(9 \mathrm{~A})$ & 3888 & 10376 & 7615 & 49 \\
\hline $\mathrm{H}(11 \mathrm{~A})$ & 4516 & 5153 & 7452 & 63 \\
\hline $\mathrm{H}(11 \mathrm{~B})$ & 6365 & 9687 & 7437 & 43 \\
\hline $\mathrm{H}(12 \mathrm{~A})$ & 2982 & 3382 & 6901 & 77 \\
\hline $\mathrm{H}(12 \mathrm{~B})$ & 2858 & 4434 & 6558 & 77 \\
\hline
\end{tabular}




\begin{tabular}{|c|c|c|c|c|}
\hline $\mathrm{H}(12 \mathrm{C})$ & 7536 & 7987 & 8174 & 56 \\
\hline $\mathrm{H}(12 \mathrm{D})$ & 7622 & 9098 & 8419 & 56 \\
\hline $\mathrm{H}(13 \mathrm{~A})$ & 4845 & 3364 & 6204 & 86 \\
\hline $\mathrm{H}(13 \mathrm{~B})$ & 5582 & 4392 & 6481 & 86 \\
\hline $\mathrm{H}(13 \mathrm{C})$ & 4904 & 9115 & 8453 & 57 \\
\hline $\mathrm{H}(13 \mathrm{D})$ & 5572 & 8113 & 8800 & 57 \\
\hline $\mathrm{H}(14 \mathrm{~A})$ & 5941 & 2545 & 7106 & 58 \\
\hline $\mathrm{H}(14 \mathrm{~B})$ & 4618 & 7198 & 7878 & 51 \\
\hline $\mathrm{H}(15 \mathrm{~A})$ & 9133 & 4008 & 10856 & 79 \\
\hline $\mathrm{H}(15 \mathrm{~B})$ & 7601 & 4274 & 10313 & 79 \\
\hline $\mathrm{H}(15 \mathrm{C})$ & 9349 & 4588 & 10219 & 79 \\
\hline $\mathrm{H}(15 \mathrm{D})$ & 1664 & 9612 & 4231 & 77 \\
\hline $\mathrm{H}(15 \mathrm{E})$ & 3106 & 9676 & 4844 & 77 \\
\hline $\mathrm{H}(15 \mathrm{~F})$ & 1351 & 10024 & 4907 & 77 \\
\hline $\mathrm{H}(16 \mathrm{~A})$ & 10397 & 1053 & 8465 & 86 \\
\hline $\mathrm{H}(16 \mathrm{~B})$ & 8999 & 1729 & 8080 & 86 \\
\hline $\mathrm{H}(16 \mathrm{C})$ & 8648 & 1011 & 8635 & 86 \\
\hline $\mathrm{H}(16 \mathrm{D})$ & -752 & 6323 & 6221 & 86 \\
\hline $\mathrm{H}(16 \mathrm{E})$ & 737 & 6821 & 6690 & 86 \\
\hline $\mathrm{H}(16 \mathrm{~F})$ & 1028 & 6136 & 6109 & 86 \\
\hline $\mathrm{H}(17 \mathrm{~A})$ & 1966 & 4994 & 7658 & 159 \\
\hline $\mathrm{H}(17 \mathrm{~B})$ & 3228 & 4852 & 8323 & 159 \\
\hline $\mathrm{H}(17 \mathrm{C})$ & 2361 & 3925 & 7948 & 159 \\
\hline $\mathrm{H}(17 \mathrm{D})$ & 8862 & 9107 & 7293 & 82 \\
\hline $\mathrm{H}(17 \mathrm{E})$ & 7593 & 9029 & 6625 & 82 \\
\hline $\mathrm{H}(17 \mathrm{~F})$ & 8090 & 8075 & 7055 & 82 \\
\hline $\mathrm{H}(18 \mathrm{~A})$ & 8106 & 3004 & 6614 & 121 \\
\hline $\mathrm{H}(18 \mathrm{~B})$ & 8700 & 2887 & 7380 & 121 \\
\hline $\mathrm{H}(18 \mathrm{C})$ & 8415 & 3950 & 7066 & 121 \\
\hline $\mathrm{H}(18 \mathrm{D})$ & 2493 & 7581 & 8381 & 104 \\
\hline $\mathrm{H}(18 \mathrm{E})$ & 1862 & 7482 & 7616 & 104 \\
\hline
\end{tabular}




\begin{tabular}{lrrrr}
$\mathrm{H}(18 \mathrm{~F})$ & 2107 & 8537 & 7943 & 104 \\
$\mathrm{H}(20 \mathrm{~A})$ & 6406 & 422 & 9035 & 62 \\
$\mathrm{H}(20 \mathrm{~B})$ & 3073 & 5324 & 5594 & 48 \\
$\mathrm{H}(21 \mathrm{~A})$ & 6163 & -719 & 9826 & 78 \\
$\mathrm{H}(21 \mathrm{~B})$ & 3120 & 4410 & 4662 & 67 \\
$\mathrm{H}(22 \mathrm{~A})$ & 5041 & -277 & 10725 & 65 \\
$\mathrm{H}(22 \mathrm{~B})$ & 4356 & 5046 & 3850 & 77 \\
$\mathrm{H}(23 \mathrm{~A})$ & 4480 & 1394 & 10871 & 65 \\
$\mathrm{H}(23 \mathrm{~B})$ & 5542 & 6606 & 3983 & 61 \\
$\mathrm{H}(24 \mathrm{~A})$ & 4955 & 2551 & 10110 & 58 \\
$\mathrm{H}(24 \mathrm{~B})$ & 5367 & 7523 & 4892 & 54 \\
& & & & \\
\hline
\end{tabular}


Table 1. Crystal data and structure refinement for $\mathrm{Pt}((\mathrm{R}, \mathrm{R})-\mathrm{Me}-\mathrm{Duphos})(\mathrm{Ph})(\mathrm{Br})$, glu215.

Identification code

Empirical formula

Formula weight

Temperature

Wavelength

Crystal system

Space group

Unit cell dimensions

Volume

Z

Density (calculated)

Absorption coefficient

$\mathrm{F}(000)$

Crystal size

Theta range for data collection

Index ranges

Reflections collected

Independent reflections

Completeness to theta $=28.22^{\circ}$

Absorption correction

Max. and min. transmission

Refinement method

Data / restraints / parameters

Goodness-of-fit on $\mathrm{F}^{2}$

Final $\mathrm{R}$ indices [I $>2 \operatorname{sigma}(\mathrm{I})]$

$\mathrm{R}$ indices (all data)

Absolute structure parameter

Largest diff. peak and hole glu215

C24 H33 Br P2 Pt

658.44

213(2) K

$0.71073 \AA$

Orthorhombic

P2(1)2(1)2(1)

$\mathrm{a}=10.701(2) \AA$

$\alpha=90^{\circ}$.

$\mathrm{b}=13.890(3) \AA$ $\beta=90^{\circ}$.

$\mathrm{c}=16.873(3) \AA$ $\gamma=90^{\circ}$.
4

$1.744 \mathrm{Mg} / \mathrm{m}^{3}$

$7.323 \mathrm{~mm}^{-1}$

1280

$0.30 \times 0.20 \times 0.10 \mathrm{~mm}^{3}$

1.90 to $28.22^{\circ}$.

$-13 \leq \mathrm{h} \leq 12,-18 \leq \mathrm{k} \leq 18,-21 \leq 1 \leq 22$

16313

$5792[\mathrm{R}(\mathrm{int})=0.0397]$

$96.4 \%$

None

0.5279 and 0.2173

Full-matrix least-squares on $\mathrm{F}^{2}$

$5792 / 0 / 253$

0.893

$\mathrm{R} 1=0.0236, \mathrm{wR} 2=0.0494$

$\mathrm{R} 1=0.0254, \mathrm{wR} 2=0.0499$

$-0.002(5)$

0.783 and -0.647 e. $\AA^{-3}$ 
Table 2. Atomic coordinates $\left(\mathrm{x} 10^{4}\right)$ and equivalent isotropic displacement parameters $\left(\AA^{2} \mathrm{x}\right.$ $\left.10^{3}\right)$ for $\mathrm{Pt}((\mathrm{R}, \mathrm{R})-\mathrm{Me}-\mathrm{Duphos})(\mathrm{Ph})(\mathrm{Br})$, glu215. $\mathrm{U}(\mathrm{eq})$ is defined as one third of the trace of the orthogonalized Uij tensor.

\begin{tabular}{|c|c|c|c|c|}
\hline & $\mathrm{X}$ & $\mathrm{y}$ & z & $\mathrm{U}(\mathrm{eq})$ \\
\hline $\operatorname{Pt}(1)$ & $6837(1)$ & $91(1)$ & $8900(1)$ & $24(1)$ \\
\hline $\operatorname{Br}(1)$ & $5768(1)$ & $-650(1)$ & $10060(1)$ & $35(1)$ \\
\hline $\mathrm{P}(1)$ & $5033(1)$ & $832(1)$ & $8528(1)$ & $26(1)$ \\
\hline $\mathrm{P}(2)$ & $7674(1)$ & $677(1)$ & $7809(1)$ & $24(1)$ \\
\hline$C(1)$ & $5203(5)$ & 2053(4) & $9879(3)$ & $61(2)$ \\
\hline$C(2)$ & $4359(5)$ & 1731(3) & $9211(3)$ & $45(1)$ \\
\hline $\mathrm{C}(3)$ & $3121(5)$ & $1279(4)$ & $9477(3)$ & $60(2)$ \\
\hline$C(4)$ & $2558(4)$ & $781(5)$ & $8757(3)$ & $61(2)$ \\
\hline$C(5)$ & $3569(4)$ & $140(3)$ & $8410(3)$ & $40(1)$ \\
\hline$C(6)$ & $3329(5)$ & $-262(4)$ & $7593(3)$ & $57(1)$ \\
\hline$C(7)$ & $8994(5)$ & $2453(3)$ & $7975(3)$ & $47(1)$ \\
\hline $\mathrm{C}(8)$ & $9132(4)$ & 1371(3) & $7907(3)$ & $32(1)$ \\
\hline $\mathrm{C}(9)$ & $9967(4)$ & 1021(3) & $7231(3)$ & $36(1)$ \\
\hline$C(10)$ & $9695(4)$ & $-47(3)$ & $7112(3)$ & $36(1)$ \\
\hline $\mathrm{C}(11)$ & $8271(3)$ & $-144(3)$ & $7039(2)$ & $29(1)$ \\
\hline$C(12)$ & $7732(4)$ & $-1151(3)$ & $7043(3)$ & $41(1)$ \\
\hline$C(13)$ & $8527(4)$ & $-392(3)$ & $9336(3)$ & $34(1)$ \\
\hline$C(14)$ & $9118(4)$ & 173(4) & $9904(3)$ & $45(1)$ \\
\hline$C(15)$ & $10246(5)$ & $-82(5)$ & $10245(3)$ & $63(2)$ \\
\hline$C(16)$ & $10824(5)$ & $-905(5)$ & $10041(4)$ & $69(2)$ \\
\hline$C(17)$ & 10293(6) & $-1489(5)$ & $9484(4)$ & $66(2)$ \\
\hline $\mathrm{C}(18)$ & $9151(5)$ & $-1241(4)$ & $9130(3)$ & $52(1)$ \\
\hline$C(19)$ & $5332(4)$ & $1492(3)$ & $7613(2)$ & $27(1)$ \\
\hline$C(20)$ & $4436(4)$ & $2058(3)$ & $7240(3)$ & $36(1)$ \\
\hline $\mathrm{C}(21)$ & $4726(4)$ & 2541(3) & $6549(3)$ & $39(1)$ \\
\hline$C(22)$ & $5901(4)$ & $2446(3)$ & $6215(3)$ & $39(1)$ \\
\hline
\end{tabular}


C(23)

C(24)
6794(4)

6525(4)
1882(3)

6583(2)

1415(3)
7289(2)

33(1)

28(1) 
Table 3. Bond lengths $[\AA]$ and angles [deg] for $\mathrm{Pt}((\mathrm{R}, \mathrm{R})-\mathrm{Me}-\mathrm{Duphos})(\mathrm{Ph})(\mathrm{Br})$, glu215.

\begin{tabular}{|c|c|c|c|}
\hline $\operatorname{Pt}(1)-\mathrm{C}(13)$ & $2.065(4)$ & $\mathrm{C}(8)-\mathrm{C}(9)$ & $1.528(6)$ \\
\hline $\mathrm{Pt}(1)-\mathrm{P}(2)$ & $2.2028(11)$ & $\mathrm{C}(9)-\mathrm{C}(10)$ & $1.524(6)$ \\
\hline $\mathrm{Pt}(1)-\mathrm{P}(1)$ & $2.2755(11)$ & $\mathrm{C}(10)-\mathrm{C}(11)$ & $1.534(5)$ \\
\hline $\operatorname{Pt}(1)-\operatorname{Br}(1)$ & $2.4900(5)$ & $\mathrm{C}(11)-\mathrm{C}(12)$ & $1.513(5)$ \\
\hline $\mathrm{P}(1)-\mathrm{C}(19)$ & $1.823(4)$ & $\mathrm{C}(13)-\mathrm{C}(18)$ & $1.399(6)$ \\
\hline $\mathrm{P}(1)-\mathrm{C}(2)$ & $1.847(5)$ & $\mathrm{C}(13)-\mathrm{C}(14)$ & $1.391(6)$ \\
\hline $\mathrm{P}(1)-\mathrm{C}(5)$ & $1.849(4)$ & $\mathrm{C}(14)-\mathrm{C}(15)$ & $1.383(6)$ \\
\hline $\mathrm{P}(2)-\mathrm{C}(24)$ & $1.827(4)$ & $C(15)-C(16)$ & $1.344(8)$ \\
\hline $\mathrm{P}(2)-\mathrm{C}(8)$ & $1.841(4)$ & $\mathrm{C}(16)-\mathrm{C}(17)$ & $1.366(9)$ \\
\hline $\mathrm{P}(2)-\mathrm{C}(11)$ & $1.843(4)$ & $\mathrm{C}(17)-\mathrm{C}(18)$ & $1.403(7)$ \\
\hline $\mathrm{C}(1)-\mathrm{C}(2)$ & $1.512(7)$ & $\mathrm{C}(19)-\mathrm{C}(24)$ & $1.393(6)$ \\
\hline$C(2)-C(3)$ & $1.534(7)$ & $C(19)-C(20)$ & $1.390(5)$ \\
\hline $\mathrm{C}(3)-\mathrm{C}(4)$ & $1.522(8)$ & $\mathrm{C}(20)-\mathrm{C}(21)$ & $1.380(6)$ \\
\hline$C(4)-C(5)$ & $1.518(6)$ & $\mathrm{C}(21)-\mathrm{C}(22)$ & $1.384(6)$ \\
\hline$C(5)-C(6)$ & $1.510(7)$ & $C(22)-C(23)$ & $1.383(6)$ \\
\hline$C(7)-C(8)$ & $1.516(6)$ & $C(23)-C(24)$ & $1.386(5)$ \\
\hline $\mathrm{C}(13)-\mathrm{Pt}(1)-\mathrm{P}(2)$ & $93.51(12)$ & $\mathrm{C}(24)-\mathrm{P}(2)-\mathrm{C}(8)$ & $108.63(18)$ \\
\hline $\mathrm{C}(13)-\operatorname{Pt}(1)-\mathrm{P}(1)$ & $171.26(13)$ & $C(24)-P(2)-C(11)$ & $103.98(18)$ \\
\hline$P(2)-P t(1)-P(1)$ & $87.00(4)$ & $\mathrm{C}(8)-\mathrm{P}(2)-\mathrm{C}(11)$ & $95.33(18)$ \\
\hline$C(13)-\operatorname{Pt}(1)-\operatorname{Br}(1)$ & $89.30(11)$ & $\mathrm{C}(24)-\mathrm{P}(2)-\mathrm{Pt}(1)$ & $109.57(14)$ \\
\hline $\mathrm{P}(2)-\operatorname{Pt}(1)-\operatorname{Br}(1)$ & $175.14(3)$ & $\mathrm{C}(8)-\mathrm{P}(2)-\mathrm{Pt}(1)$ & $117.61(14)$ \\
\hline $\mathrm{P}(1)-\mathrm{Pt}(1)-\operatorname{Br}(1)$ & $90.84(3)$ & $\mathrm{C}(11)-\mathrm{P}(2)-\mathrm{Pt}(1)$ & $120.15(12)$ \\
\hline $\mathrm{C}(19)-\mathrm{P}(1)-\mathrm{C}(2)$ & $104.8(2)$ & $\mathrm{C}(1)-\mathrm{C}(2)-\mathrm{C}(3)$ & $114.8(4)$ \\
\hline $\mathrm{C}(19)-\mathrm{P}(1)-\mathrm{C}(5)$ & $108.6(2)$ & $\mathrm{C}(1)-\mathrm{C}(2)-\mathrm{P}(1)$ & $115.6(3)$ \\
\hline$C(2)-P(1)-C(5)$ & $95.0(2)$ & $\mathrm{C}(3)-\mathrm{C}(2)-\mathrm{P}(1)$ & $104.0(3)$ \\
\hline $\mathrm{C}(19)-\mathrm{P}(1)-\mathrm{Pt}(1)$ & $108.20(14)$ & $\mathrm{C}(4)-\mathrm{C}(3)-\mathrm{C}(2)$ & $107.1(4)$ \\
\hline $\mathrm{C}(2)-\mathrm{P}(1)-\mathrm{Pt}(1)$ & $117.68(17)$ & $\mathrm{C}(3)-\mathrm{C}(4)-\mathrm{C}(5)$ & $107.0(4)$ \\
\hline $\mathrm{C}(5)-\mathrm{P}(1)-\mathrm{Pt}(1)$ & $120.91(15)$ & $\mathrm{C}(6)-\mathrm{C}(5)-\mathrm{C}(4)$ & $116.6(4)$ \\
\hline
\end{tabular}




\begin{tabular}{|c|c|}
\hline$C(6)-C(5)-P(1)$ & $115.8(3)$ \\
\hline $\mathrm{C}(4)-\mathrm{C}(5)-\mathrm{P}(1)$ & $104.9(3)$ \\
\hline $\mathrm{C}(7)-\mathrm{C}(8)-\mathrm{C}(9)$ & $115.5(4)$ \\
\hline $\mathrm{C}(7)-\mathrm{C}(8)-\mathrm{P}(2)$ & $116.3(3)$ \\
\hline $\mathrm{C}(9)-\mathrm{C}(8)-\mathrm{P}(2)$ & $105.2(3)$ \\
\hline $\mathrm{C}(10)-\mathrm{C}(9)-\mathrm{C}(8)$ & 107.2(3) \\
\hline $\mathrm{C}(9)-\mathrm{C}(10)-\mathrm{C}(11)$ & $106.6(3)$ \\
\hline $\mathrm{C}(12)-\mathrm{C}(11)-\mathrm{C}(10)$ & $117.4(4)$ \\
\hline $\mathrm{C}(12)-\mathrm{C}(11)-\mathrm{P}(2)$ & $115.9(3)$ \\
\hline $\mathrm{C}(10)-\mathrm{C}(11)-\mathrm{P}(2)$ & $103.5(3)$ \\
\hline $\mathrm{C}(18)-\mathrm{C}(13)-\mathrm{C}(14)$ & $115.5(4)$ \\
\hline $\mathrm{C}(18)-\mathrm{C}(13)-\mathrm{Pt}(1)$ & $127.1(4)$ \\
\hline $\mathrm{C}(14)-\mathrm{C}(13)-\mathrm{Pt}(1)$ & $117.4(3)$ \\
\hline $\mathrm{C}(15)-\mathrm{C}(14)-\mathrm{C}(13)$ & $122.6(5)$ \\
\hline$C(16)-C(15)-C(14)$ & $120.8(6)$ \\
\hline $\mathrm{C}(17)-\mathrm{C}(16)-\mathrm{C}(15)$ & $119.3(5)$ \\
\hline$C(16)-C(17)-C(18)$ & $120.7(6)$ \\
\hline$C(13)-C(18)-C(17)$ & $121.1(6)$ \\
\hline$C(24)-C(19)-C(20)$ & $119.8(4)$ \\
\hline $\mathrm{C}(24)-\mathrm{C}(19)-\mathrm{P}(1)$ & 117.1(3) \\
\hline $\mathrm{C}(20)-\mathrm{C}(19)-\mathrm{P}(1)$ & $123.1(3)$ \\
\hline $\mathrm{C}(21)-\mathrm{C}(20)-\mathrm{C}(19)$ & $120.1(4)$ \\
\hline$C(22)-C(21)-C(20)$ & $120.2(4)$ \\
\hline$C(21)-C(22)-C(23)$ & $119.9(4)$ \\
\hline $\mathrm{C}(22)-\mathrm{C}(23)-\mathrm{C}(24)$ & $120.4(4)$ \\
\hline$C(23)-C(24)-C(19)$ & $119.5(4)$ \\
\hline $\mathrm{C}(23)-\mathrm{C}(24)-\mathrm{P}(2)$ & $122.4(3)$ \\
\hline $\mathrm{C}(19)-\mathrm{C}(24)-\mathrm{P}(2)$ & $118.1(3)$ \\
\hline
\end{tabular}

Symmetry transformations used to generate equivalent atoms: 
Table 4. Anisotropic displacement parameters $\left(\AA^{2} \times 10^{3}\right)$ for $\mathrm{Pt}((\mathrm{R}, \mathrm{R})-\mathrm{Me}-\mathrm{Duphos})(\mathrm{Ph})(\mathrm{Br})$, glu215. The anisotropic displacement factor exponent takes the form: $-2 \pi^{2}\left[h^{2} a^{* 2} U^{11}+\ldots+2 h k a^{*} b^{*} U^{12}\right]$

\begin{tabular}{|c|c|c|c|c|c|c|}
\hline & $\mathrm{U}^{11}$ & $\mathrm{U}^{22}$ & $\mathrm{U}^{33}$ & $\mathrm{U}^{23}$ & $\mathrm{U}^{13}$ & $\mathrm{U}^{12}$ \\
\hline $\operatorname{Pt}(1)$ & $27(1)$ & $25(1)$ & $20(1)$ & $2(1)$ & $-1(1)$ & 1(1) \\
\hline $\operatorname{Br}(1)$ & $43(1)$ & $42(1)$ & 21(1) & $4(1)$ & $1(1)$ & $-6(1)$ \\
\hline $\mathrm{P}(1)$ & $25(1)$ & $31(1)$ & $23(1)$ & 1(1) & $0(1)$ & 1(1) \\
\hline $\mathrm{P}(2)$ & $24(1)$ & $26(1)$ & $22(1)$ & $3(1)$ & $-1(1)$ & $0(1)$ \\
\hline$C(1)$ & $82(4)$ & $55(3)$ & $45(3)$ & $-24(3)$ & $1(3)$ & $11(3)$ \\
\hline$C(2)$ & $48(3)$ & $50(3)$ & $36(3)$ & $-3(2)$ & $7(2)$ & $16(2)$ \\
\hline$C(3)$ & 41(3) & $98(4)$ & $42(3)$ & $8(3)$ & $17(3)$ & $18(3)$ \\
\hline$C(4)$ & $29(3)$ & $101(4)$ & $53(4)$ & 21(3) & $6(3)$ & 3(3) \\
\hline$C(5)$ & $31(2)$ & $47(2)$ & $42(3)$ & $13(2)$ & $-7(2)$ & $-7(2)$ \\
\hline$C(6)$ & $49(3)$ & $57(3)$ & $65(4)$ & $-1(3)$ & $-16(3)$ & $-17(2)$ \\
\hline$C(7)$ & $55(3)$ & $35(2)$ & $51(3)$ & $-4(2)$ & $-7(3)$ & $-12(2)$ \\
\hline$C(8)$ & $29(2)$ & $37(2)$ & $29(2)$ & $3(2)$ & $-4(2)$ & $-11(2)$ \\
\hline$C(9)$ & $26(2)$ & $45(2)$ & $35(3)$ & $10(2)$ & $-1(2)$ & $-7(2)$ \\
\hline$C(10)$ & $32(2)$ & $42(2)$ & $35(2)$ & $0(2)$ & $1(2)$ & $7(2)$ \\
\hline$C(11)$ & $28(2)$ & $32(2)$ & $26(2)$ & $-1(2)$ & $3(2)$ & $4(2)$ \\
\hline$C(12)$ & $45(3)$ & $37(2)$ & $39(3)$ & $-6(2)$ & $3(2)$ & $-3(2)$ \\
\hline$C(13)$ & $36(2)$ & $40(2)$ & $27(2)$ & $11(2)$ & $2(2)$ & $3(2)$ \\
\hline$C(14)$ & $43(3)$ & $52(3)$ & 41(3) & $14(2)$ & $-11(2)$ & $-5(2)$ \\
\hline$C(15)$ & $47(3)$ & $87(4)$ & $56(4)$ & $21(3)$ & $-22(3)$ & $-20(3)$ \\
\hline$C(16)$ & $33(3)$ & $100(5)$ & $74(4)$ & $48(4)$ & $-6(3)$ & $10(3)$ \\
\hline$C(17)$ & $54(4)$ & $82(4)$ & $64(4)$ & $29(4)$ & 11(3) & $31(3)$ \\
\hline$C(18)$ & $56(3)$ & $59(3)$ & $42(3)$ & $12(2)$ & $-3(3)$ & $18(3)$ \\
\hline$C(19)$ & $34(2)$ & $26(2)$ & $23(2)$ & 1(2) & $1(2)$ & 1(2) \\
\hline$C(20)$ & $30(2)$ & $41(2)$ & $36(2)$ & $0(2)$ & $-1(2)$ & $9(2)$ \\
\hline $\mathrm{C}(21)$ & $43(3)$ & $32(2)$ & 41(3) & $10(2)$ & $-9(2)$ & $7(2)$ \\
\hline$C(22)$ & $52(3)$ & $36(2)$ & $28(2)$ & $9(2)$ & $-5(2)$ & $-2(2)$ \\
\hline
\end{tabular}


C(23)

36(2)

28(2)

5(2)

0(2)

1(2)

C(24)

25(2)

25(2)

0(2)

$-4(2)$

$-1(2)$ 
Table 5. Hydrogen coordinates ( $\left.\times 10^{4}\right)$ and isotropic displacement parameters $\left(\AA^{2} \times 10^{3}\right)$ for $\operatorname{Pt}((\mathrm{R}, \mathrm{R})-\mathrm{Me}-\mathrm{Duphos})(\mathrm{Ph})(\mathrm{Br})$, glu215.

\begin{tabular}{|c|c|c|c|c|}
\hline & $\mathrm{x}$ & $\mathrm{y}$ & $\mathrm{z}$ & $\mathrm{U}(\mathrm{eq})$ \\
\hline $\mathrm{H}(1 \mathrm{~A})$ & 4779 & 2538 & 10193 & 91 \\
\hline $\mathrm{H}(1 \mathrm{~B})$ & 5966 & 2322 & 9661 & 91 \\
\hline $\mathrm{H}(1 \mathrm{C})$ & 5406 & 1505 & 10211 & 91 \\
\hline $\mathrm{H}(2 \mathrm{~A})$ & 4153 & 2309 & 8893 & 54 \\
\hline $\mathrm{H}(3 \mathrm{~A})$ & 3268 & 811 & 9902 & 72 \\
\hline $\mathrm{H}(3 \mathrm{~B})$ & 2552 & 1777 & 9674 & 72 \\
\hline $\mathrm{H}(4 \mathrm{~A})$ & 2288 & 1259 & 8365 & 73 \\
\hline $\mathrm{H}(4 \mathrm{~B})$ & 1832 & 395 & 8913 & 73 \\
\hline $\mathrm{H}(5 \mathrm{~A})$ & 3638 & -422 & 8769 & 48 \\
\hline $\mathrm{H}(6 \mathrm{~A})$ & 2537 & -602 & 7590 & 85 \\
\hline $\mathrm{H}(6 \mathrm{~B})$ & 3995 & -703 & 7451 & 85 \\
\hline $\mathrm{H}(6 \mathrm{C})$ & 3300 & 261 & 7212 & 85 \\
\hline $\mathrm{H}(7 \mathrm{~A})$ & 9810 & 2743 & 8049 & 71 \\
\hline $\mathrm{H}(7 \mathrm{~B})$ & 8466 & 2606 & 8426 & 71 \\
\hline $\mathrm{H}(7 \mathrm{C})$ & 8616 & 2704 & 7495 & 71 \\
\hline $\mathrm{H}(8 \mathrm{~A})$ & 9532 & 1152 & 8404 & 38 \\
\hline $\mathrm{H}(9 \mathrm{~A})$ & 9784 & 1380 & 6745 & 43 \\
\hline $\mathrm{H}(9 \mathrm{~B})$ & 10849 & 1116 & 7366 & 43 \\
\hline $\mathrm{H}(10 \mathrm{~A})$ & 10001 & -421 & 7565 & 43 \\
\hline $\mathrm{H}(10 \mathrm{~B})$ & 10105 & -283 & 6630 & 43 \\
\hline $\mathrm{H}(11 \mathrm{~A})$ & 8040 & 145 & 6523 & 34 \\
\hline $\mathrm{H}(12 \mathrm{~A})$ & 8094 & -1520 & 6612 & 61 \\
\hline $\mathrm{H}(12 \mathrm{~B})$ & 6833 & -1117 & 6975 & 61 \\
\hline $\mathrm{H}(12 \mathrm{C})$ & 7923 & -1461 & 7543 & 61 \\
\hline $\mathrm{H}(14 \mathrm{~A})$ & 8735 & 750 & 10063 & 54 \\
\hline
\end{tabular}




\begin{tabular}{lrrrr}
$\mathrm{H}(15 \mathrm{~A})$ & 10613 & 325 & 10624 & 76 \\
$\mathrm{H}(16 \mathrm{~A})$ & 11586 & -1076 & 10279 & 83 \\
$\mathrm{H}(17 \mathrm{~A})$ & 10697 & -2062 & 9336 & 80 \\
$\mathrm{H}(18 \mathrm{~A})$ & 8799 & -1653 & 8748 & 63 \\
$\mathrm{H}(20 \mathrm{~A})$ & 3631 & 2111 & 7459 & 43 \\
$\mathrm{H}(21 \mathrm{~A})$ & 4125 & 2935 & 6305 & 46 \\
$\mathrm{H}(22 \mathrm{~A})$ & 6091 & 2765 & 5739 & 46 \\
$\mathrm{H}(23 \mathrm{~A})$ & 7589 & 1814 & 6352 & 40 \\
& & & & \\
\hline
\end{tabular}


Table 1. Crystal data and structure refinement for [Pt((R,R)-Me-Duphos)(Ph)(PMe(Is) $\left.\left(\mathrm{CH}_{2} \mathrm{Ph}\right)\right]\left[\mathrm{BF}_{4}\right]$, GLU214.

Identification code

Empirical formula

Formula weight

Temperature

Wavelength

Crystal system

Space group

Unit cell dimensions

Volume

Z

Density (calculated)

Absorption coefficient

$\mathrm{F}(000)$

Crystal size

Theta range for data collection

Index ranges

Reflections collected

Independent reflections

Completeness to theta $=28.22^{\circ}$

Absorption correction

Max. and min. transmission

Refinement method

Data / restraints / parameters

Goodness-of-fit on $\mathrm{F}^{2}$

Final R indices [I $>2 \operatorname{sigma}(\mathrm{I})]$

$\mathrm{R}$ indices (all data)

Absolute structure parameter glu214

$\mathrm{C}_{47} \mathrm{H}_{66} \mathrm{~B} \mathrm{~F}_{4} \mathrm{P}_{3} \mathrm{Pt}$

1005.81

213(2) K

$0.71073 \AA$

Orthorhombic

P2(1)2(1)2(1)

$$
\begin{array}{ll}
\mathrm{a}=12.9514(16) \AA & \alpha=90^{\circ} . \\
\mathrm{b}=17.462(2) \AA & \beta=90^{\circ} . \\
\mathrm{c}=21.291(3) \AA & \gamma=90^{\circ} .
\end{array}
$$

4814.9(10) $\AA^{3}$

4

$1.388 \mathrm{Mg} / \mathrm{m}^{3}$

$3.059 \mathrm{~mm}^{-1}$

2048

$0.35 \times 0.20 \times 0.20 \mathrm{~mm}^{3}$

1.84 to $28.22^{\circ}$.

$-15 \leq \mathrm{h} \leq 16,-22 \leq \mathrm{k} \leq 21,-28 \leq \mathrm{l} \leq 28$

36407

10747 [R(int) $=0.0298]$

$97.0 \%$

Semi-empirical from equivalents

0.5450 and 0.3892

Full-matrix least-squares on $\mathrm{F}^{2}$

10747 / 18 / 577

0.991

$\mathrm{R} 1=0.0227, \mathrm{wR} 2=0.0494$

$\mathrm{R} 1=0.0247, \mathrm{wR} 2=0.0497$

$-0.005(3)$ 
Table 2. Atomic coordinates ( $\left.\times 10^{4}\right)$ and equivalent isotropic displacement parameters $\left(\AA^{2} \times 10^{3}\right)$ for $\left[\mathrm{Pt}((\mathrm{R}, \mathrm{R})-\mathrm{Me}-\mathrm{Duphos})(\mathrm{Ph})\left(\mathrm{PMe}(\mathrm{Is})\left(\mathrm{CH}_{2} \mathrm{Ph}\right)\right]\left[\mathrm{BF}_{4}\right], \mathrm{GLU} 214\right.$. U(eq) is defined as one third of the trace of the orthogonalized $\mathrm{U}^{\mathrm{ij}}$ tensor.

\begin{tabular}{|c|c|c|c|c|}
\hline & $\mathrm{x}$ & $\mathrm{y}$ & $\mathrm{z}$ & $\mathrm{U}(\mathrm{eq})$ \\
\hline $\operatorname{Pt}(1)$ & $686(1)$ & 9390(1) & $2368(1)$ & $28(1)$ \\
\hline $\mathrm{P}(1)$ & $71(1)$ & $9063(1)$ & $1386(1)$ & $33(1)$ \\
\hline $\mathrm{P}(2)$ & $2276(1)$ & 9134(1) & $1952(1)$ & $41(1)$ \\
\hline $\mathrm{P}(3)$ & $-802(1)$ & 9773(1) & 2901(1) & $30(1)$ \\
\hline $\mathrm{F}(1)$ & $6778(2)$ & $7437(2)$ & $1544(1)$ & $95(1)$ \\
\hline $\mathrm{F}(2)$ & $6345(3)$ & $8643(2)$ & 1694(2) & $155(2)$ \\
\hline $\mathrm{F}(3)$ & $5272(3)$ & $7859(2)$ & $1205(2)$ & $121(1)$ \\
\hline $\mathrm{F}(4)$ & $5486(2)$ & 7733(2) & $2219(2)$ & $114(1)$ \\
\hline $\mathrm{B}(1)$ & $5977(4)$ & 7928(4) & 1664(3) & $77(2)$ \\
\hline$C(1)$ & $-465(3)$ & 9803(2) & $843(2)$ & $48(1)$ \\
\hline$C(2)$ & $-1581(3)$ & $9572(2)$ & $739(2)$ & $57(1)$ \\
\hline$C(3)$ & $-1638(3)$ & $8692(2)$ & $725(2)$ & $49(1)$ \\
\hline$C(4)$ & $-1027(2)$ & $8400(2)$ & 1299(1) & $36(1)$ \\
\hline$C(5)$ & $-295(4)$ & $10630(2)$ & $1049(2)$ & $70(1)$ \\
\hline$C(6)$ & $-751(3)$ & 7551(2) & $1288(2)$ & $45(1)$ \\
\hline$C(7)$ & 1141(3) & $8659(2)$ & $936(1)$ & $38(1)$ \\
\hline$C(8)$ & $993(3)$ & $8345(2)$ & $334(1)$ & $47(1)$ \\
\hline$C(9)$ & 1818(3) & $8039(2)$ & $7(2)$ & $56(1)$ \\
\hline $\mathrm{C}(10)$ & $2780(4)$ & $8059(2)$ & $268(2)$ & $60(1)$ \\
\hline $\mathrm{C}(11)$ & 2949(3) & $8381(2)$ & $856(2)$ & $57(1)$ \\
\hline$C(12)$ & 2112(3) & $8678(2)$ & 1191(1) & $41(1)$ \\
\hline$C(13)$ & $3095(3)$ & 9998(2) & $1853(2)$ & $73(1)$ \\
\hline$C(14)$ & $4204(16)$ & $9840(20)$ & $2075(17)$ & $79(6)$ \\
\hline$C(15)$ & $4010(18)$ & $9214(12)$ & $2546(13)$ & $84(9)$ \\
\hline
\end{tabular}




\begin{tabular}{|c|c|c|c|c|}
\hline $\mathrm{C}(17)$ & $3210(30)$ & $10220(20)$ & $1186(10)$ & $143(18)$ \\
\hline $\mathrm{C}(14 \mathrm{~A})$ & $4124(13)$ & $9680(16)$ & $2133(12)$ & $144(13)$ \\
\hline$C(15 \mathrm{~A})$ & $3967(13)$ & $9157(12)$ & 2681(11) & $128(10)$ \\
\hline$C(17 A)$ & $3080(20)$ & $10392(11)$ & $1198(5)$ & $82(5)$ \\
\hline$C(16)$ & $3263(3)$ & $8573(3)$ & $2351(2)$ & $68(1)$ \\
\hline$C(18)$ & 2898(4) & 7956(3) & 2793(2) & $96(2)$ \\
\hline$C(19)$ & $-940(2)$ & $9334(2)$ & $3680(1)$ & $37(1)$ \\
\hline$C(20)$ & $-1104(3)$ & $8523(2)$ & $3669(2)$ & $46(1)$ \\
\hline $\mathrm{C}(21)$ & $-1269(4)$ & $8152(2)$ & $4238(2)$ & $67(1)$ \\
\hline$C(22)$ & $-1289(5)$ & $8521(3)$ & $4806(2)$ & $81(2)$ \\
\hline$C(23)$ & $-1115(4)$ & $9301(2)$ & $4800(2)$ & $65(1)$ \\
\hline$C(24)$ & $-918(3)$ & $9712(2)$ & $4263(2)$ & $42(1)$ \\
\hline$C(25)$ & $-1056(3)$ & $8019(2)$ & $3088(2)$ & $46(1)$ \\
\hline$C(26)$ & $-109(4)$ & $7503(2)$ & $3106(2)$ & $67(1)$ \\
\hline$C(27)$ & $-2049(4)$ & $7549(2)$ & $3000(2)$ & $80(1)$ \\
\hline$C(28)$ & $-1279(18)$ & $8074(15)$ & $5435(9)$ & $115(12)$ \\
\hline$C(29)$ & $-190(20)$ & $8010(20)$ & $5717(14)$ & $450(50)$ \\
\hline$C(30)$ & $-2170(20)$ & $8366(16)$ & $5820(12)$ & $210(20)$ \\
\hline$C(28 \mathrm{~A})$ & $-1592(16)$ & $8064(8)$ & $5396(6)$ & 133(9) \\
\hline$C(29 A)$ & $-789(18)$ & $7435(12)$ & $5514(7)$ & $250(15)$ \\
\hline$C(30 A)$ & $-2676(12)$ & 7751(8) & $5360(6)$ & $168(8)$ \\
\hline$C(31)$ & $-698(3)$ & $10572(2)$ & $4339(1)$ & $47(1)$ \\
\hline$C(32)$ & $-90(4)$ & 10767(3) & $4925(2)$ & $75(1)$ \\
\hline$C(33)$ & $-1698(3)$ & $11034(2)$ & $4330(2)$ & $61(1)$ \\
\hline$C(34)$ & $-2102(2)$ & $9654(2)$ & $2561(2)$ & $40(1)$ \\
\hline$C(35)$ & $-2953(3)$ & $10039(2)$ & $2934(2)$ & $48(1)$ \\
\hline$C(36)$ & $-3292(3)$ & $10772(2)$ & $2766(2)$ & $66(1)$ \\
\hline$C(37)$ & $-4065(4)$ & $11140(3)$ & $3109(3)$ & $85(2)$ \\
\hline$C(38)$ & $-4480(20)$ & $10788(8)$ & $3631(11)$ & $112(16)$ \\
\hline$C(38 \mathrm{~A})$ & $-4507(10)$ & 10761(8) & $3605(6)$ & 101(10) \\
\hline C(39) & $-4197(4)$ & $10040(4)$ & $3776(2)$ & $88(2)$ \\
\hline
\end{tabular}




$\begin{array}{lrrrr}\mathrm{C}(40) & -3413(3) & 9672(3) & 3436(2) & 62(1) \\ \mathrm{C}(41) & -725(3) & 10817(1) & 2892(2) & 40(1) \\ \mathrm{C}(42) & 1418(2) & 9710(2) & 3204(1) & 35(1) \\ \mathrm{C}(43) & 1504(3) & 9231(2) & 3727(2) & 44(1) \\ \mathrm{C}(44) & 2048(3) & 9444(2) & 4256(2) & 57(1) \\ \mathrm{C}(45) & 2521(3) & 10147(2) & 4282(2) & 64(1) \\ \mathrm{C}(46) & 2438(3) & 10642(2) & 3781(2) & 53(1) \\ \mathrm{C}(47) & 1892(2) & 10431(2) & 3253(2) & 40(1) \\ \end{array}$


Table 3. Bond lengths $[\AA]$ and angles $[\mathrm{deg}]$ for $\left[\mathrm{Pt}((\mathrm{R}, \mathrm{R})-\mathrm{Me}-\mathrm{Duphos})(\mathrm{Ph})\left(\mathrm{PMe}(\mathrm{Is})\left(\mathrm{CH}_{2} \mathrm{Ph}\right)\right]\left[\mathrm{BF}_{4}\right]\right.$, GLU214.

\begin{tabular}{|c|c|c|c|}
\hline $\operatorname{Pt}(1)-C(42)$ & $2.093(3)$ & $C(13)-C(17)$ & $1.479(18)$ \\
\hline $\operatorname{Pt}(1)-\mathrm{P}(2)$ & $2.2853(9)$ & $C(13)-C(14)$ & $1.536(16)$ \\
\hline $\operatorname{Pt}(1)-\mathrm{P}(1)$ & $2.3085(7)$ & $C(13)-C(17 A)$ & $1.554(11)$ \\
\hline $\mathrm{Pt}(1)-\mathrm{P}(3)$ & $2.3338(8)$ & $C(13)-C(14 A)$ & $1.562(15)$ \\
\hline $\mathrm{P}(1)-\mathrm{C}(7)$ & $1.826(3)$ & $\mathrm{C}(14)-\mathrm{C}(15)$ & $1.506(18)$ \\
\hline $\mathrm{P}(1)-\mathrm{C}(4)$ & $1.842(3)$ & $C(15)-C(16)$ & $1.536(17)$ \\
\hline $\mathrm{P}(1)-\mathrm{C}(1)$ & $1.867(3)$ & $C(14 \mathrm{~A})-\mathrm{C}(15 \mathrm{~A})$ & $1.495(16)$ \\
\hline $\mathrm{P}(2)-\mathrm{C}(12)$ & $1.817(3)$ & $C(15 A)-C(16)$ & $1.537(11)$ \\
\hline $\mathrm{P}(2)-\mathrm{C}(16)$ & $1.822(4)$ & $C(16)-C(18)$ & $1.507(7)$ \\
\hline $\mathrm{P}(2)-\mathrm{C}(13)$ & $1.856(4)$ & $C(19)-C(24)$ & $1.406(4)$ \\
\hline $\mathrm{P}(3)-\mathrm{C}(41)$ & $1.825(3)$ & $C(19)-C(20)$ & $1.432(4)$ \\
\hline $\mathrm{P}(3)-\mathrm{C}(19)$ & $1.836(3)$ & $C(20)-C(21)$ & $1.389(5)$ \\
\hline $\mathrm{P}(3)-\mathrm{C}(34)$ & $1.845(3)$ & $C(20)-C(25)$ & $1.519(5)$ \\
\hline $\mathrm{F}(1)-\mathrm{B}(1)$ & $1.370(6)$ & $C(21)-C(22)$ & $1.370(6)$ \\
\hline $\mathrm{F}(2)-\mathrm{B}(1)$ & $1.339(6)$ & $C(22)-C(23)$ & $1.381(6)$ \\
\hline $\mathrm{F}(3)-\mathrm{B}(1)$ & $1.342(7)$ & $\mathrm{C}(22)-\mathrm{C}(28 \mathrm{~A})$ & $1.539(12)$ \\
\hline $\mathrm{F}(4)-\mathrm{B}(1)$ & $1.384(7)$ & $C(22)-C(28)$ & $1.551(16)$ \\
\hline$C(1)-C(2)$ & $1.517(5)$ & $C(23)-C(24)$ & $1.373(5)$ \\
\hline$C(1)-C(5)$ & $1.525(5)$ & $\mathrm{C}(24)-\mathrm{C}(31)$ & $1.537(4)$ \\
\hline$C(2)-C(3)$ & $1.538(5)$ & $C(25)-C(26)$ & $1.522(5)$ \\
\hline$C(3)-C(4)$ & $1.543(4)$ & $C(25)-C(27)$ & $1.537(5)$ \\
\hline$C(4)-C(6)$ & $1.526(4)$ & $C(28)-C(30)$ & $1.500(18)$ \\
\hline$C(7)-C(12)$ & $1.371(5)$ & $C(28)-C(29)$ & $1.541(19)$ \\
\hline$C(7)-C(8)$ & $1.407(4)$ & $C(28 \mathrm{~A})-\mathrm{C}(30 \mathrm{~A})$ & $1.509(17)$ \\
\hline $\mathrm{C}(8)-\mathrm{C}(9)$ & $1.384(5)$ & $C(28 \mathrm{~A})-\mathrm{C}(29 \mathrm{~A})$ & $1.534(15)$ \\
\hline $\mathrm{C}(9)-\mathrm{C}(10)$ & $1.365(6)$ & $\mathrm{C}(31)-\mathrm{C}(32)$ & $1.515(5)$ \\
\hline $\mathrm{C}(10)-\mathrm{C}(11)$ & $1.391(5)$ & $\mathrm{C}(31)-\mathrm{C}(33)$ & $1.526(5)$ \\
\hline $\mathrm{C}(11)-\mathrm{C}(12)$ & $1.397(5)$ & $\mathrm{C}(34)-\mathrm{C}(35)$ & $1.516(4)$ \\
\hline
\end{tabular}




\begin{tabular}{|c|c|c|c|}
\hline$C(35)-C(40)$ & $1.380(6)$ & $\mathrm{C}(39)-\mathrm{C}(40)$ & $1.403(6)$ \\
\hline$C(35)-C(36)$ & $1.400(5)$ & $\mathrm{C}(42)-\mathrm{C}(43)$ & $1.397(4)$ \\
\hline$C(36)-C(37)$ & $1.396(6)$ & $\mathrm{C}(42)-\mathrm{C}(47)$ & $1.404(4)$ \\
\hline $\mathrm{C}(37)-\mathrm{C}(38 \mathrm{~A})$ & $1.372(12)$ & $\mathrm{C}(43)-\mathrm{C}(44)$ & $1.379(5)$ \\
\hline $\mathrm{C}(37)-\mathrm{C}(38)$ & $1.377(17)$ & $\mathrm{C}(44)-\mathrm{C}(45)$ & $1.374(6)$ \\
\hline $\mathrm{C}(38)-\mathrm{C}(39)$ & $1.390(15)$ & $\mathrm{C}(45)-\mathrm{C}(46)$ & $1.376(6)$ \\
\hline C(38A)-C(39) & $1.371(12)$ & $\mathrm{C}(46)-\mathrm{C}(47)$ & $1.380(4)$ \\
\hline $\mathrm{C}(42)-\mathrm{Pt}(1)-\mathrm{P}(2)$ & $88.47(9)$ & $\mathrm{C}(34)-\mathrm{P}(3)-\mathrm{Pt}(1)$ & $122.04(10)$ \\
\hline $\mathrm{C}(42)-\mathrm{Pt}(1)-\mathrm{P}(1)$ & 172.94(9) & $\mathrm{F}(2)-\mathrm{B}(1)-\mathrm{F}(3)$ & $111.2(6)$ \\
\hline $\mathrm{P}(2)-\mathrm{Pt}(1)-\mathrm{P}(1)$ & $84.93(3)$ & $\mathrm{F}(2)-\mathrm{B}(1)-\mathrm{F}(1)$ & $108.9(4)$ \\
\hline $\mathrm{C}(42)-\mathrm{Pt}(1)-\mathrm{P}(3)$ & $83.35(8)$ & $\mathrm{F}(3)-\mathrm{B}(1)-\mathrm{F}(1)$ & $108.8(4)$ \\
\hline $\mathrm{P}(2)-\mathrm{Pt}(1)-\mathrm{P}(3)$ & $171.14(3)$ & $\mathrm{F}(2)-\mathrm{B}(1)-\mathrm{F}(4)$ & $110.7(5)$ \\
\hline $\mathrm{P}(1)-\mathrm{Pt}(1)-\mathrm{P}(3)$ & $103.09(3)$ & $\mathrm{F}(3)-\mathrm{B}(1)-\mathrm{F}(4)$ & $106.7(4)$ \\
\hline $\mathrm{C}(7)-\mathrm{P}(1)-\mathrm{C}(4)$ & $106.87(14)$ & $\mathrm{F}(1)-\mathrm{B}(1)-\mathrm{F}(4)$ & $110.6(5)$ \\
\hline $\mathrm{C}(7)-\mathrm{P}(1)-\mathrm{C}(1)$ & $102.92(16)$ & $C(2)-C(1)-C(5)$ & $115.5(3)$ \\
\hline $\mathrm{C}(4)-\mathrm{P}(1)-\mathrm{C}(1)$ & $94.89(15)$ & $\mathrm{C}(2)-\mathrm{C}(1)-\mathrm{P}(1)$ & $105.1(2)$ \\
\hline $\mathrm{C}(7)-\mathrm{P}(1)-\mathrm{Pt}(1)$ & $108.00(11)$ & $\mathrm{C}(5)-\mathrm{C}(1)-\mathrm{P}(1)$ & $115.1(2)$ \\
\hline $\mathrm{C}(4)-\mathrm{P}(1)-\mathrm{Pt}(1)$ & $120.85(10)$ & $\mathrm{C}(1)-\mathrm{C}(2)-\mathrm{C}(3)$ & $108.3(3)$ \\
\hline $\mathrm{C}(1)-\mathrm{P}(1)-\mathrm{Pt}(1)$ & $121.19(10)$ & $\mathrm{C}(2)-\mathrm{C}(3)-\mathrm{C}(4)$ & $106.8(3)$ \\
\hline $\mathrm{C}(12)-\mathrm{P}(2)-\mathrm{C}(16)$ & $105.14(17)$ & $\mathrm{C}(6)-\mathrm{C}(4)-\mathrm{C}(3)$ & $115.4(3)$ \\
\hline $\mathrm{C}(12)-\mathrm{P}(2)-\mathrm{C}(13)$ & $108.73(18)$ & $\mathrm{C}(6)-\mathrm{C}(4)-\mathrm{P}(1)$ & $115.6(2)$ \\
\hline $\mathrm{C}(16)-\mathrm{P}(2)-\mathrm{C}(13)$ & $95.1(2)$ & $\mathrm{C}(3)-\mathrm{C}(4)-\mathrm{P}(1)$ & $105.6(2)$ \\
\hline $\mathrm{C}(12)-\mathrm{P}(2)-\mathrm{Pt}(1)$ & $109.04(12)$ & $C(12)-C(7)-C(8)$ & $119.7(3)$ \\
\hline $\mathrm{C}(16)-\mathrm{P}(2)-\mathrm{Pt}(1)$ & $123.83(15)$ & $\mathrm{C}(12)-\mathrm{C}(7)-\mathrm{P}(1)$ & $118.6(2)$ \\
\hline $\mathrm{C}(13)-\mathrm{P}(2)-\mathrm{Pt}(1)$ & $113.67(14)$ & $\mathrm{C}(8)-\mathrm{C}(7)-\mathrm{P}(1)$ & $121.7(3)$ \\
\hline $\mathrm{C}(41)-\mathrm{P}(3)-\mathrm{C}(19)$ & $115.60(14)$ & $\mathrm{C}(9)-\mathrm{C}(8)-\mathrm{C}(7)$ & $120.3(4)$ \\
\hline $\mathrm{C}(41)-\mathrm{P}(3)-\mathrm{C}(34)$ & $99.07(15)$ & $\mathrm{C}(10)-\mathrm{C}(9)-\mathrm{C}(8)$ & $119.3(3)$ \\
\hline $\mathrm{C}(19)-\mathrm{P}(3)-\mathrm{C}(34)$ & $102.62(14)$ & $C(9)-C(10)-C(11)$ & $121.4(4)$ \\
\hline $\mathrm{C}(41)-\mathrm{P}(3)-\mathrm{Pt}(1)$ & $103.65(11)$ & $C(10)-C(11)-C(12)$ & $119.2(4)$ \\
\hline C(19)-P(3)-Pt(1) & $113.59(10)$ & $\mathrm{C}(7)-\mathrm{C}(12)-\mathrm{C}(11)$ & $120.0(3)$ \\
\hline
\end{tabular}




\begin{tabular}{|c|c|c|c|}
\hline $\mathrm{C}(7)-\mathrm{C}(12)-\mathrm{P}(2)$ & $118.1(2)$ & $C(23)-C(22)-C(28 A)$ & $124.0(7)$ \\
\hline $\mathrm{C}(11)-\mathrm{C}(12)-\mathrm{P}(2)$ & $121.9(3)$ & $\mathrm{C}(21)-\mathrm{C}(22)-\mathrm{C}(28)$ & $121.7(11)$ \\
\hline $\mathrm{C}(17)-\mathrm{C}(13)-\mathrm{C}(14)$ & $105(2)$ & $\mathrm{C}(23)-\mathrm{C}(22)-\mathrm{C}(28)$ & $120.1(11)$ \\
\hline $\mathrm{C}(17)-\mathrm{C}(13)-\mathrm{C}(17 \mathrm{~A})$ & $13(2)$ & $\mathrm{C}(28 \mathrm{~A})-\mathrm{C}(22)-\mathrm{C}(28)$ & $15.5(13)$ \\
\hline $\mathrm{C}(14)-\mathrm{C}(13)-\mathrm{C}(17 \mathrm{~A})$ & $111.8(16)$ & $\mathrm{C}(24)-\mathrm{C}(23)-\mathrm{C}(22)$ & $123.5(3)$ \\
\hline $\mathrm{C}(17)-\mathrm{C}(13)-\mathrm{C}(14 \mathrm{~A})$ & $112(2)$ & $\mathrm{C}(23)-\mathrm{C}(24)-\mathrm{C}(19)$ & 119.1(3) \\
\hline $\mathrm{C}(14)-\mathrm{C}(13)-\mathrm{C}(14 \mathrm{~A})$ & $12.0(19)$ & $\mathrm{C}(23)-\mathrm{C}(24)-\mathrm{C}(31)$ & $117.2(3)$ \\
\hline $\mathrm{C}(17 \mathrm{~A})-\mathrm{C}(13)-\mathrm{C}(14 \mathrm{~A})$ & $121.0(14)$ & $C(19)-C(24)-C(31)$ & $123.7(3)$ \\
\hline $\mathrm{C}(17)-\mathrm{C}(13)-\mathrm{P}(2)$ & $112.4(15)$ & $C(20)-C(25)-C(26)$ & $110.8(3)$ \\
\hline $\mathrm{C}(14)-\mathrm{C}(13)-\mathrm{P}(2)$ & $110.8(13)$ & $C(20)-C(25)-C(27)$ & $112.0(3)$ \\
\hline $\mathrm{C}(17 \mathrm{~A})-\mathrm{C}(13)-\mathrm{P}(2)$ & $116.9(8)$ & $C(26)-C(25)-C(27)$ & $111.2(3)$ \\
\hline $\mathrm{C}(14 \mathrm{~A})-\mathrm{C}(13)-\mathrm{P}(2)$ & $99.0(10)$ & $\mathrm{C}(30)-\mathrm{C}(28)-\mathrm{C}(29)$ & $121(3)$ \\
\hline $\mathrm{C}(15)-\mathrm{C}(14)-\mathrm{C}(13)$ & $100.3(18)$ & $\mathrm{C}(30)-\mathrm{C}(28)-\mathrm{C}(22)$ & $107.2(14)$ \\
\hline $\mathrm{C}(14)-\mathrm{C}(15)-\mathrm{C}(16)$ & $117(2)$ & $\mathrm{C}(29)-\mathrm{C}(28)-\mathrm{C}(22)$ & $112.3(14)$ \\
\hline$C(15 A)-C(14 A)-C(13)$ & $113.5(15)$ & $\mathrm{C}(30 \mathrm{~A})-\mathrm{C}(28 \mathrm{~A})-\mathrm{C}(29 \mathrm{~A})$ & $112.3(14)$ \\
\hline$C(14 A)-C(15 A)-C(16)$ & $97.5(16)$ & $C(30 A)-C(28 A)-C(22)$ & $112.7(12)$ \\
\hline$C(18)-C(16)-C(15)$ & $123.4(11)$ & $C(29 A)-C(28 A)-C(22)$ & $109.4(10)$ \\
\hline $\mathrm{C}(18)-\mathrm{C}(16)-\mathrm{C}(15 \mathrm{~A})$ & $112.0(10)$ & $\mathrm{C}(32)-\mathrm{C}(31)-\mathrm{C}(33)$ & 109.4(3) \\
\hline$C(15)-C(16)-C(15 A)$ & $11.6(18)$ & $\mathrm{C}(32)-\mathrm{C}(31)-\mathrm{C}(24)$ & $113.7(3)$ \\
\hline $\mathrm{C}(18)-\mathrm{C}(16)-\mathrm{P}(2)$ & 117.1(3) & $\mathrm{C}(33)-\mathrm{C}(31)-\mathrm{C}(24)$ & $111.0(3)$ \\
\hline$C(15)-C(16)-P(2)$ & $100.2(10)$ & $\mathrm{C}(35)-\mathrm{C}(34)-\mathrm{P}(3)$ & $114.1(2)$ \\
\hline$C(15 A)-C(16)-P(2)$ & $105.8(9)$ & $\mathrm{C}(40)-\mathrm{C}(35)-\mathrm{C}(36)$ & $119.2(4)$ \\
\hline$C(24)-C(19)-C(20)$ & $118.8(3)$ & $\mathrm{C}(40)-\mathrm{C}(35)-\mathrm{C}(34)$ & $120.9(3)$ \\
\hline $\mathrm{C}(24)-\mathrm{C}(19)-\mathrm{P}(3)$ & $126.8(2)$ & $\mathrm{C}(36)-\mathrm{C}(35)-\mathrm{C}(34)$ & $119.9(3)$ \\
\hline$C(20)-C(19)-P(3)$ & $114.4(2)$ & $C(37)-C(36)-C(35)$ & $120.8(5)$ \\
\hline $\mathrm{C}(21)-\mathrm{C}(20)-\mathrm{C}(19)$ & $117.9(3)$ & $\mathrm{C}(38 \mathrm{~A})-\mathrm{C}(37)-\mathrm{C}(38)$ & $3.4(18)$ \\
\hline$C(21)-C(20)-C(25)$ & $116.6(3)$ & $\mathrm{C}(38 \mathrm{~A})-\mathrm{C}(37)-\mathrm{C}(36)$ & $118.7(6)$ \\
\hline $\mathrm{C}(19)-\mathrm{C}(20)-\mathrm{C}(25)$ & $125.4(3)$ & $\mathrm{C}(38)-\mathrm{C}(37)-\mathrm{C}(36)$ & $119.6(10)$ \\
\hline$C(22)-C(21)-C(20)$ & $123.6(4)$ & $\mathrm{C}(37)-\mathrm{C}(38)-\mathrm{C}(39)$ & $119.9(16)$ \\
\hline $\mathrm{C}(21)-\mathrm{C}(22)-\mathrm{C}(23)$ & $117.0(3)$ & $\mathrm{C}(39)-\mathrm{C}(38 \mathrm{~A})-\mathrm{C}(37)$ & $121.7(7)$ \\
\hline $\mathrm{C}(21)-\mathrm{C}(22)-\mathrm{C}(28 \mathrm{~A})$ & $118.8(7)$ & $\mathrm{C}(38 \mathrm{~A})-\mathrm{C}(39)-\mathrm{C}(38)$ & $3.3(19)$ \\
\hline
\end{tabular}




$\begin{array}{ll}\mathrm{C}(38 \mathrm{~A})-\mathrm{C}(39)-\mathrm{C}(40) & 119.7(6) \\ \mathrm{C}(38)-\mathrm{C}(39)-\mathrm{C}(40) & 120.2(10) \\ \mathrm{C}(35)-\mathrm{C}(40)-\mathrm{C}(39) & 120.0(5) \\ \mathrm{C}(43)-\mathrm{C}(42)-\mathrm{C}(47) & 116.3(3) \\ \mathrm{C}(43)-\mathrm{C}(42)-\mathrm{Pt}(1) & 123.6(2) \\ \mathrm{C}(47)-\mathrm{C}(42)-\mathrm{Pt}(1) & 120.0(2) \\ \mathrm{C}(44)-\mathrm{C}(43)-\mathrm{C}(42) & 122.0(3) \\ \mathrm{C}(45)-\mathrm{C}(44)-\mathrm{C}(43) & 120.1(4) \\ \mathrm{C}(44)-\mathrm{C}(45)-\mathrm{C}(46) & 119.7(3) \\ \mathrm{C}(45)-\mathrm{C}(46)-\mathrm{C}(47) & 120.3(4) \\ \mathrm{C}(46)-\mathrm{C}(47)-\mathrm{C}(42) & 121.6(3)\end{array}$

Symmetry transformations used to generate equivalent atoms: 
Table 4. Anisotropic displacement parameters $\left(\AA^{2} \times 10^{3}\right)$ for $\left[\mathrm{Pt}((\mathrm{R}, \mathrm{R})-\mathrm{Me}-\mathrm{Duphos})(\mathrm{Ph})\left(\mathrm{PMe}(\mathrm{Is})\left(\mathrm{CH}_{2} \mathrm{Ph}^{2}\right)\right]\left[\mathrm{BF}_{4}\right]\right.$, GLU214. The anisotropic displacement factor exponent takes the form: $-2 \pi^{2}\left[h^{2} a^{* 2} U^{11}+\ldots+2 h k a^{*} b^{*} U^{12}\right]$

\begin{tabular}{|c|c|c|c|c|c|c|}
\hline & $\mathrm{U}^{11}$ & $\mathrm{U}^{22}$ & $\mathrm{U}^{33}$ & $\mathrm{U}^{23}$ & $\mathrm{U}^{13}$ & $\mathrm{U}^{12}$ \\
\hline $\operatorname{Pt}(1)$ & $30(1)$ & $31(1)$ & $25(1)$ & $-5(1)$ & 1(1) & $-1(1)$ \\
\hline $\mathrm{P}(1)$ & $40(1)$ & $33(1)$ & $27(1)$ & $-3(1)$ & $-3(1)$ & $-4(1)$ \\
\hline $\mathrm{P}(2)$ & $34(1)$ & $51(1)$ & $40(1)$ & $-17(1)$ & $4(1)$ & $-3(1)$ \\
\hline $\mathrm{P}(3)$ & $32(1)$ & $29(1)$ & $31(1)$ & $-2(1)$ & $4(1)$ & $0(1)$ \\
\hline $\mathrm{F}(1)$ & $65(2)$ & $131(2)$ & $88(2)$ & $-31(2)$ & $2(1)$ & $31(2)$ \\
\hline $\mathrm{F}(2)$ & $105(3)$ & $106(3)$ & $254(5)$ & $-79(3)$ & $65(3)$ & $-40(2)$ \\
\hline $\mathrm{F}(3)$ & $74(2)$ & $151(3)$ & $136(3)$ & $1(2)$ & $-30(2)$ & $-4(2)$ \\
\hline $\mathrm{F}(4)$ & $83(2)$ & $140(3)$ & $118(3)$ & $-12(2)$ & $39(2)$ & $15(2)$ \\
\hline $\mathrm{B}(1)$ & $39(3)$ & $92(4)$ & $101(4)$ & $-33(3)$ & $7(3)$ & $0(3)$ \\
\hline$C(1)$ & $75(3)$ & $36(2)$ & $35(2)$ & $2(1)$ & $-12(2)$ & $-3(2)$ \\
\hline$C(2)$ & $78(3)$ & $44(2)$ & $50(2)$ & $0(2)$ & $-27(2)$ & $13(2)$ \\
\hline$C(3)$ & $51(2)$ & $49(2)$ & $45(2)$ & $-9(2)$ & $-22(2)$ & $-1(2)$ \\
\hline$C(4)$ & $35(2)$ & $36(2)$ & $36(2)$ & $-2(1)$ & $-9(1)$ & $-3(1)$ \\
\hline$C(5)$ & $113(4)$ & $39(2)$ & $58(2)$ & $0(2)$ & $-19(2)$ & $-1(2)$ \\
\hline$C(6)$ & $49(2)$ & $38(2)$ & $47(2)$ & $-2(1)$ & $-4(2)$ & $-4(2)$ \\
\hline$C(7)$ & $49(2)$ & $35(2)$ & $29(1)$ & $-4(1)$ & 11(1) & $-9(1)$ \\
\hline$C(8)$ & $66(3)$ & $42(2)$ & $34(2)$ & $-3(1)$ & $6(2)$ & $-12(2)$ \\
\hline $\mathrm{C}(9)$ & $93(3)$ & $43(2)$ & $33(2)$ & $-10(1)$ & $24(2)$ & $-19(2)$ \\
\hline $\mathrm{C}(10)$ & $77(3)$ & $51(2)$ & $52(2)$ & $-18(2)$ & $37(2)$ & $-13(2)$ \\
\hline $\mathrm{C}(11)$ & $53(2)$ & $56(2)$ & $61(2)$ & $-12(2)$ & $19(2)$ & $-9(2)$ \\
\hline $\mathrm{C}(12)$ & $49(2)$ & $39(2)$ & $36(2)$ & $-10(1)$ & $15(2)$ & $-9(2)$ \\
\hline$C(13)$ & $53(3)$ & 73(3) & $92(3)$ & $-42(2)$ & $31(2)$ & $-30(2)$ \\
\hline $\mathrm{C}(14)$ & $33(10)$ & $90(11)$ & $112(16)$ & $-30(11)$ & $3(10)$ & $-24(10)$ \\
\hline$C(15)$ & $52(13)$ & $140(20)$ & 61(10) & $-41(10)$ & $-10(7)$ & $52(13)$ \\
\hline$C(17)$ & $120(20)$ & $80(20)$ & $230(30)$ & $-43(15)$ & $100(20)$ & $-62(17)$ \\
\hline$C(14 A)$ & $55(9)$ & $180(20)$ & $200(20)$ & $-127(19)$ & $48(12)$ & $-63(11)$ \\
\hline
\end{tabular}




\begin{tabular}{|c|c|c|c|c|c|c|}
\hline$C(15 A)$ & $37(7)$ & $210(20)$ & $139(17)$ & $-110(14)$ & $-43(8)$ & $11(8)$ \\
\hline$C(17 \mathrm{~A})$ & $120(11)$ & $47(6)$ & $80(7)$ & $-11(4)$ & $60(7)$ & $-22(5)$ \\
\hline$C(16)$ & $48(2)$ & $97(3)$ & $58(2)$ & $-33(2)$ & $-10(2)$ & $27(2)$ \\
\hline $\mathrm{C}(18)$ & $108(4)$ & $120(4)$ & $58(3)$ & $-14(3)$ & $-16(3)$ & $78(4)$ \\
\hline$C(19)$ & $37(2)$ & $40(2)$ & $32(1)$ & $3(1)$ & $8(1)$ & $-2(1)$ \\
\hline$C(20)$ & $55(2)$ & $42(2)$ & $40(2)$ & $3(1)$ & $9(2)$ & $0(2)$ \\
\hline$C(21)$ & $104(4)$ & $45(2)$ & $51(2)$ & $12(2)$ & $18(2)$ & $-7(2)$ \\
\hline$C(22)$ & $139(5)$ & $65(3)$ & $40(2)$ & $11(2)$ & $24(3)$ & $-3(3)$ \\
\hline$C(23)$ & $100(3)$ & $61(2)$ & $35(2)$ & $0(2)$ & $22(2)$ & $6(2)$ \\
\hline $\mathrm{C}(24)$ & $45(2)$ & $46(2)$ & $36(2)$ & $-1(1)$ & $9(1)$ & $2(1)$ \\
\hline$C(25)$ & $62(2)$ & $30(2)$ & $45(2)$ & $1(1)$ & $3(2)$ & $-6(1)$ \\
\hline$C(26)$ & 104(4) & $42(2)$ & $54(2)$ & $-11(2)$ & $-7(2)$ & $19(2)$ \\
\hline$C(27)$ & $93(4)$ & $61(2)$ & $86(3)$ & $1(2)$ & $-4(3)$ & $-31(2)$ \\
\hline$C(28)$ & $200(20)$ & $110(20)$ & $38(10)$ & $11(11)$ & $19(12)$ & $-79(19)$ \\
\hline$C(29)$ & $580(80)$ & $460(60)$ & $300(40)$ & $320(40)$ & $-370(50)$ & $-430(60)$ \\
\hline$C(30)$ & $310(40)$ & $180(30)$ & $130(20)$ & $120(20)$ & $140(20)$ & $150(30)$ \\
\hline $\mathrm{C}(28 \mathrm{~A})$ & $260(20)$ & $66(10)$ & $71(10)$ & $24(7)$ & $84(12)$ & $22(12)$ \\
\hline$C(29 A)$ & $350(30)$ & $250(20)$ & $150(13)$ & $163(15)$ & $138(16)$ & $170(20)$ \\
\hline$C(30 A)$ & $268(19)$ & $110(9)$ & $126(11)$ & $16(8)$ & $136(12)$ & $-32(10)$ \\
\hline$C(31)$ & $50(2)$ & $53(2)$ & $37(2)$ & $-13(1)$ & $11(2)$ & $-11(2)$ \\
\hline$C(32)$ & $86(3)$ & $97(3)$ & $44(2)$ & $-18(2)$ & $1(2)$ & $-22(3)$ \\
\hline $\mathrm{C}(33)$ & $65(3)$ & $51(2)$ & $67(2)$ & $-18(2)$ & $27(2)$ & $-4(2)$ \\
\hline C(34) & $30(2)$ & $44(1)$ & $45(2)$ & $-1(1)$ & $-6(1)$ & $-1(1)$ \\
\hline$C(35)$ & $29(2)$ & $52(2)$ & $63(2)$ & $-16(2)$ & $-1(2)$ & $-3(1)$ \\
\hline$C(36)$ & $42(2)$ & $64(2)$ & 93(3) & $-18(2)$ & $-8(2)$ & $12(2)$ \\
\hline $\mathrm{C}(37)$ & $55(3)$ & $77(3)$ & $123(5)$ & $-33(3)$ & $-5(3)$ & $20(2)$ \\
\hline $\mathrm{C}(38)$ & $90(20)$ & $64(14)$ & $180(40)$ & $-63(17)$ & $-50(19)$ & $2(12)$ \\
\hline$C(38 \mathrm{~A})$ & $26(7)$ & $170(20)$ & $109(13)$ & $-76(13)$ & $9(7)$ & $26(8)$ \\
\hline C(39) & $42(3)$ & $132(5)$ & $90(4)$ & $-43(3)$ & $18(3)$ & $-11(3)$ \\
\hline $\mathrm{C}(40)$ & $40(2)$ & $76(3)$ & $71(3)$ & $-21(2)$ & $10(2)$ & $-2(2)$ \\
\hline$C(41)$ & $47(2)$ & $29(1)$ & $43(2)$ & $-3(1)$ & $8(2)$ & $1(1)$ \\
\hline
\end{tabular}




\begin{tabular}{lllllll}
$\mathrm{C}(42)$ & $34(2)$ & $43(2)$ & $28(1)$ & $-9(1)$ & $-5(1)$ & $5(1)$ \\
$\mathrm{C}(43)$ & $51(2)$ & $38(2)$ & $41(2)$ & $-7(1)$ & $-5(1)$ & $8(1)$ \\
$\mathrm{C}(44)$ & $71(3)$ & $57(2)$ & $42(2)$ & $-6(2)$ & $-20(2)$ & $10(2)$ \\
$\mathrm{C}(45)$ & $69(3)$ & $71(3)$ & $53(2)$ & $-21(2)$ & $-29(2)$ & $11(2)$ \\
$\mathrm{C}(46)$ & $45(2)$ & $54(2)$ & $60(2)$ & $-27(2)$ & $-10(2)$ & $1(2)$ \\
$\mathrm{C}(47)$ & $39(2)$ & $40(2)$ & $42(2)$ & $-11(1)$ & $0(1)$ & $4(1)$ \\
\hline
\end{tabular}


Table 5. Hydrogen coordinates ( x 10 $)$ and isotropic displacement parameters $\left(\AA^{2} \times 10^{3}\right)$ for $\left[\mathrm{Pt}((\mathrm{R}, \mathrm{R})-\mathrm{Me}-\mathrm{Duphos})(\mathrm{Ph})\left(\mathrm{PMe}(\mathrm{Is})\left(\mathrm{CH}_{2} \mathrm{Ph}\right)\right]\left[\mathrm{BF}_{4}\right]\right.$, GLU214.

\begin{tabular}{|c|c|c|c|c|}
\hline & $\mathrm{x}$ & $\mathrm{y}$ & z & $\mathrm{U}(\mathrm{eq})$ \\
\hline $\mathrm{H}(1 \mathrm{~A})$ & -106 & 9739 & 436 & 58 \\
\hline $\mathrm{H}(2 \mathrm{~A})$ & -1833 & 9783 & 341 & 69 \\
\hline $\mathrm{H}(2 \mathrm{~B})$ & -2014 & 9771 & 1080 & 69 \\
\hline $\mathrm{H}(3 \mathrm{~A})$ & -1334 & 8495 & 336 & 58 \\
\hline $\mathrm{H}(3 \mathrm{~B})$ & -2358 & 8521 & 748 & 58 \\
\hline $\mathrm{H}(4 \mathrm{~A})$ & -1475 & 8479 & 1670 & 43 \\
\hline $\mathrm{H}(5 \mathrm{~A})$ & -478 & 10972 & 707 & 105 \\
\hline $\mathrm{H}(5 \mathrm{~B})$ & 425 & 10703 & 1158 & 105 \\
\hline $\mathrm{H}(5 \mathrm{C})$ & -724 & 10740 & 1411 & 105 \\
\hline $\mathrm{H}(6 \mathrm{~A})$ & -1379 & 7248 & 1292 & 67 \\
\hline $\mathrm{H}(6 \mathrm{~B})$ & -338 & 7426 & 1654 & 67 \\
\hline $\mathrm{H}(6 \mathrm{C})$ & -361 & 7437 & 910 & 67 \\
\hline $\mathrm{H}(8 \mathrm{~A})$ & 330 & 8343 & 154 & 57 \\
\hline $\mathrm{H}(9 \mathrm{~A})$ & 1717 & 7820 & -392 & 68 \\
\hline $\mathrm{H}(10 \mathrm{~A})$ & 3340 & 7850 & 45 & 72 \\
\hline $\mathrm{H}(11 \mathrm{~A})$ & 3618 & 8399 & 1026 & 68 \\
\hline $\mathrm{H}(13 \mathrm{~A})$ & 2800 & 10429 & 2095 & 87 \\
\hline $\mathrm{H}(14 \mathrm{~A})$ & 4645 & 9666 & 1730 & 94 \\
\hline $\mathrm{H}(14 \mathrm{~B})$ & 4513 & 10293 & 2271 & 94 \\
\hline $\mathrm{H}(15 \mathrm{~A})$ & 3744 & 9450 & 2931 & 101 \\
\hline $\mathrm{H}(15 \mathrm{~B})$ & 4676 & 8978 & 2650 & 101 \\
\hline $\mathrm{H}(17 \mathrm{~A})$ & 2536 & 10356 & 1016 & 214 \\
\hline $\mathrm{H}(17 \mathrm{~B})$ & 3495 & 9800 & 949 & 214 \\
\hline $\mathrm{H}(17 \mathrm{C})$ & 3664 & 10662 & 1156 & 214 \\
\hline $\mathrm{H}(14 \mathrm{C})$ & 4498 & 9403 & 1804 & 173 \\
\hline
\end{tabular}




\begin{tabular}{|c|c|c|c|c|}
\hline $\mathrm{H}(14 \mathrm{D})$ & 4555 & 10112 & 2266 & 173 \\
\hline $\mathrm{H}(15 \mathrm{C})$ & 3620 & 9413 & 3032 & 153 \\
\hline$H(15 D)$ & 4614 & 8927 & 2825 & 153 \\
\hline $\mathrm{H}(17 \mathrm{D})$ & 2377 & 10556 & 1103 & 123 \\
\hline $\mathrm{H}(17 \mathrm{E})$ & 3306 & 10032 & 881 & 123 \\
\hline $\mathrm{H}(17 \mathrm{~F})$ & 3530 & 10833 & 1202 & 123 \\
\hline $\mathrm{H}(16 \mathrm{~A})$ & 3625 & 8295 & 2011 & 81 \\
\hline $\mathrm{H}(18 \mathrm{~A})$ & 3485 & 7662 & 2940 & 143 \\
\hline $\mathrm{H}(18 \mathrm{~B})$ & 2421 & 7621 & 2575 & 143 \\
\hline $\mathrm{H}(18 \mathrm{C})$ & 2552 & 8189 & 3149 & 143 \\
\hline $\mathrm{H}(21 \mathrm{~A})$ & -1373 & 7620 & 4233 & 80 \\
\hline $\mathrm{H}(23 \mathrm{~A})$ & -1133 & 9566 & 5185 & 78 \\
\hline $\mathrm{H}(25 \mathrm{~A})$ & -985 & 8360 & 2720 & 55 \\
\hline $\mathrm{H}(26 \mathrm{~A})$ & 511 & 7815 & 3088 & 100 \\
\hline $\mathrm{H}(26 \mathrm{~B})$ & -122 & 7158 & 2749 & 100 \\
\hline $\mathrm{H}(26 \mathrm{C})$ & -111 & 7208 & 3492 & 100 \\
\hline $\mathrm{H}(27 \mathrm{~A})$ & -2640 & 7891 & 2995 & 120 \\
\hline $\mathrm{H}(27 \mathrm{~B})$ & -2120 & 7188 & 3343 & 120 \\
\hline $\mathrm{H}(27 \mathrm{C})$ & -2015 & 7273 & 2605 & 120 \\
\hline $\mathrm{H}(28)$ & -1471 & 7543 & 5322 & 138 \\
\hline $\mathrm{H}(29 \mathrm{~A})$ & 288 & 7823 & 5399 & 668 \\
\hline $\mathrm{H}(29 \mathrm{~B})$ & -196 & 7659 & 6069 & 668 \\
\hline $\mathrm{H}(29 \mathrm{C})$ & 39 & 8511 & 5860 & 668 \\
\hline $\mathrm{H}(30 \mathrm{~A})$ & -2765 & 8434 & 5553 & 314 \\
\hline $\mathrm{H}(30 \mathrm{~B})$ & -1980 & 8853 & 6008 & 314 \\
\hline $\mathrm{H}(30 \mathrm{C})$ & -2325 & 7999 & 6149 & 314 \\
\hline $\mathrm{H}(28 \mathrm{~A})$ & -1566 & 8421 & 5757 & 160 \\
\hline$H(29 D)$ & -104 & 7659 & 5518 & 375 \\
\hline $\mathrm{H}(29 \mathrm{E})$ & -831 & 7055 & 5183 & 375 \\
\hline $\mathrm{H}(29 \mathrm{~F})$ & -924 & 7194 & 5916 & 375 \\
\hline$H(30 D)$ & -2844 & 7494 & 5751 & 252 \\
\hline
\end{tabular}




\begin{tabular}{|c|c|c|c|c|}
\hline $\mathrm{H}(30 \mathrm{E})$ & -2724 & 7389 & 5016 & 252 \\
\hline $\mathrm{H}(30 \mathrm{~F})$ & -3158 & 8168 & 5291 & 252 \\
\hline $\mathrm{H}(31 \mathrm{~A})$ & -281 & 10735 & 3972 & 56 \\
\hline $\mathrm{H}(32 \mathrm{~A})$ & 498 & 10425 & 4961 & 113 \\
\hline $\mathrm{H}(32 \mathrm{~B})$ & -529 & 10709 & 5291 & 113 \\
\hline $\mathrm{H}(32 \mathrm{C})$ & 151 & 11292 & 4899 & 113 \\
\hline $\mathrm{H}(33 \mathrm{~A})$ & -1536 & 11576 & 4309 & 92 \\
\hline $\mathrm{H}(33 \mathrm{~B})$ & -2088 & 10930 & 4709 & 92 \\
\hline $\mathrm{H}(33 \mathrm{C})$ & -2105 & 10889 & 3966 & 92 \\
\hline $\mathrm{H}(34 \mathrm{~A})$ & -2101 & 9864 & 2134 & 47 \\
\hline $\mathrm{H}(34 \mathrm{~B})$ & -2255 & 9106 & 2530 & 47 \\
\hline $\mathrm{H}(36 \mathrm{~A})$ & -2996 & 11019 & 2417 & 80 \\
\hline $\mathrm{H}(37 \mathrm{~A})$ & -4304 & 11625 & 2985 & 102 \\
\hline $\mathrm{H}(38)$ & -4945 & 11053 & 3889 & 135 \\
\hline $\mathrm{H}(38 \mathrm{~A})$ & -5035 & 11003 & 3834 & 121 \\
\hline $\mathrm{H}(39 \mathrm{~A})$ & -4535 & 9779 & 4104 & 106 \\
\hline $\mathrm{H}(40 \mathrm{~A})$ & -3202 & 9176 & 3549 & 74 \\
\hline $\mathrm{H}(41 \mathrm{~A})$ & -1276 & 11028 & 3147 & 60 \\
\hline $\mathrm{H}(41 \mathrm{~B})$ & -793 & 10999 & 2464 & 60 \\
\hline $\mathrm{H}(41 \mathrm{C})$ & -63 & 10977 & 3060 & 60 \\
\hline $\mathrm{H}(43 \mathrm{~A})$ & 1181 & 8749 & 3718 & 52 \\
\hline $\mathrm{H}(44 \mathrm{~A})$ & 2095 & 9107 & 4599 & 68 \\
\hline $\mathrm{H}(45 \mathrm{~A})$ & 2900 & 10290 & 4640 & 77 \\
\hline $\mathrm{H}(46 \mathrm{~A})$ & 2755 & 11126 & 3800 & 63 \\
\hline $\mathrm{H}(47 \mathrm{~A})$ & 1836 & 10778 & 2917 & 48 \\
\hline
\end{tabular}


Crystal mounted on a CryoLoop ${ }^{\odot}$ with Paratone- $\mathrm{N}^{\odot}$ oil and immediately placed under a liquid stream of $\mathrm{N}_{2}$ on a Bruker SMART APEX CCD system. Data collected at $-60{ }^{\circ} \mathrm{C}$ with Mo $\mathrm{K} \alpha$ radiation and corrected for absorption using the SADABS program. Structure solved from a Patterson Fourier synthesis, developed by successive difference Fourier syntheses, and refined by full matrix least squares on all $\mathrm{F}^{2}$ data. All non-hydrogen atoms were refined as being anisotropic and hydrogen atoms were placed in calculated positions with temperature factors fixed at 1.2 or 1.5 times the equivalent isotropic $\mathrm{U}$ of the $\mathrm{C}$ atoms to which they were bonded. Carbon atoms C14, C15, C17, C28, C29 C30 and C38 were modeled for disorder over two positions in roughly $38.6 / 61.4 \%$ multiplicity. However, these $\mathrm{C}$ atoms still exhibit distortion in their thermal ellipsoids.

Although two of the fluorine atoms in the $\mathrm{BF}_{4}$ moiety exhibited high thermal parameters these atoms were not treated for disorder.

SMART, SAINT, SADABS and SHELXTL programs obtained Bruker AXS Madison, WI

CryoLoop and Paratone-N oil, Hampton Research, 34 Journey Aliso Viejo, CA 92656-3317. 
Figure S8. ORTEP diagram of $\left.(\mathrm{Pt}((\mathrm{R}, \mathrm{R})-\mathrm{Me}-\mathrm{Duphos})(\mathrm{Ph}))_{2}\left(\mu-\mathrm{CO}_{3}\right)\right]$, with hydrogen atoms omitted. One of the two independent molecules is shown.

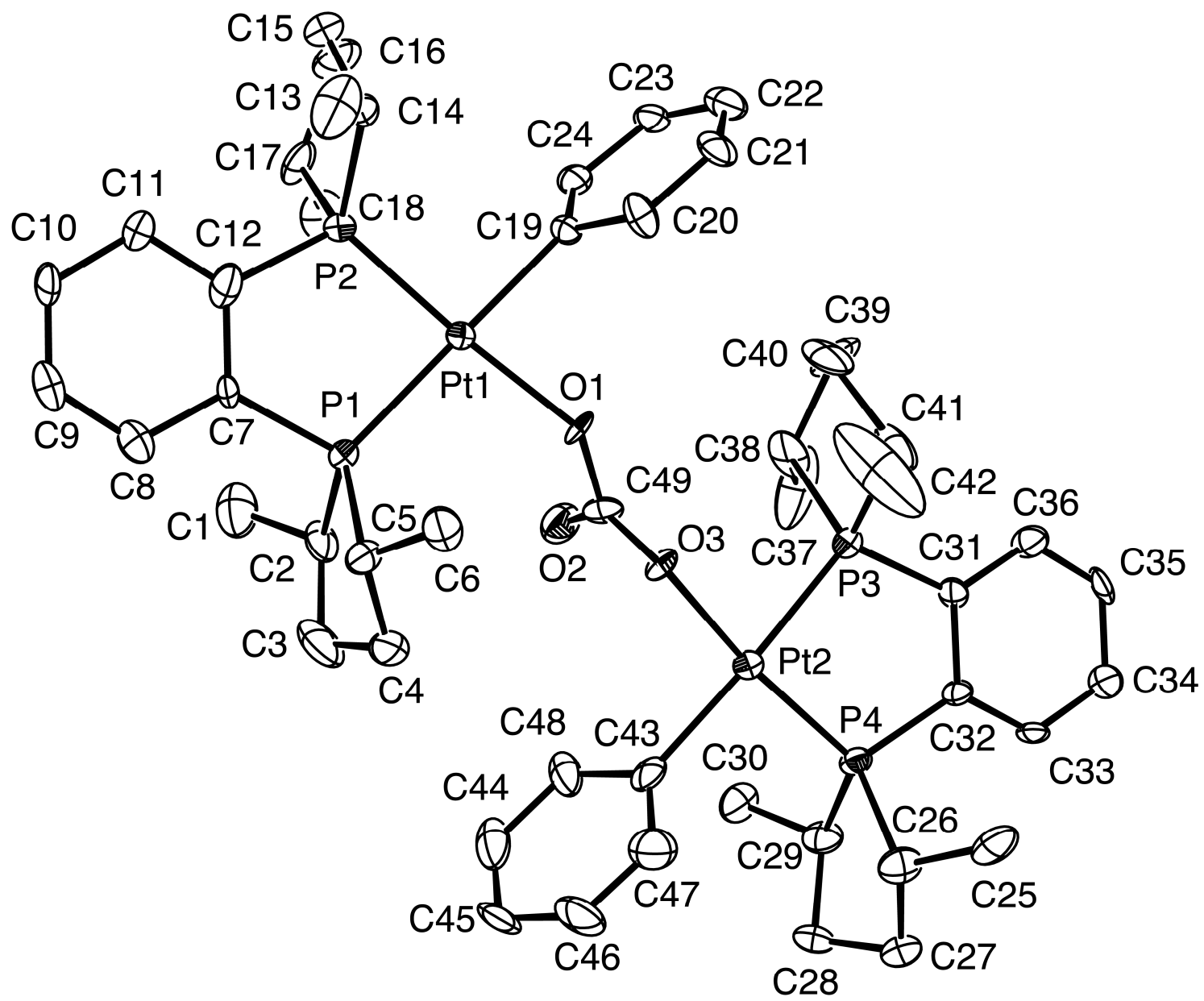


Table 1. Crystal data and structure refinement for $[\mathrm{Pt}(\mathrm{Me}-\mathrm{Duphos})(\mathrm{Ph})]_{2}\left(\mu-\mathrm{CO}_{3}\right)$, glu220.

Identification code

Empirical formula

Formula weight

Temperature

Wavelength

Crystal system

Space group

Unit cell dimensions

Volume

Z

Density (calculated)

Absorption coefficient

$\mathrm{F}(000)$

Crystal size

Theta range for data collection

Index ranges

Reflections collected

Independent reflections

Completeness to theta $=26.00^{\circ}$

Absorption correction

Max. and min. transmission

Refinement method

Data / restraints / parameters

Goodness-of-fit on $\mathrm{F}^{2}$

Final $\mathrm{R}$ indices [I $>2 \operatorname{sigma}(\mathrm{I})]$

$\mathrm{R}$ indices (all data)

Absolute structure parameter

Largest diff. peak and hole glu220

$\mathrm{C}_{49} \mathrm{H}_{66} \mathrm{O}_{3} \mathrm{P}_{4} \mathrm{Pt}_{2}$

1216.57

213(2) K

$0.71073 \AA$

Orthorhombic

P2(1)2(1)2(1)

$$
\begin{array}{ll}
\mathrm{a}=15.920(2) \AA & \alpha=90^{\circ} \\
\mathrm{b}=16.545(3) \AA & \beta=90^{\circ} \\
\mathrm{c}=40.382(6) \AA & \gamma=90^{\circ}
\end{array}
$$

$10637(3) \AA^{3}$

8

$1.519 \mathrm{~g} / \mathrm{cm}^{3}$

$5.411 \mathrm{~mm}^{-1}$

4796

$0.30 \times 0.30 \times 0.10 \mathrm{~mm}^{3}$

1.01 to $26.00^{\circ}$

$-19 \leq \mathrm{h} \leq 19,-19 \leq \mathrm{k} \leq 20,-46 \leq \mathrm{l} \leq 49$

72795

$19422[\mathrm{R}(\mathrm{int})=0.0501]$

$99.8 \%$

None

0.6137 and 0.2936

Full-matrix least-squares on $\mathrm{F}^{2}$

19422 / 0 / 1045

1.148

$\mathrm{R} 1=0.0650, \mathrm{wR} 2=0.1828$

$\mathrm{R} 1=0.0696, w \mathrm{R} 2=0.1853$

$0.068(11)$

3.469 and -2.004 e $\AA^{-3}$ 
Table 2. Atomic coordinates $\left(\mathrm{x} 10^{4}\right)$ and equivalent isotropic displacement parameters $\left(\AA^{2} \times 10^{3}\right)$ for $[\mathrm{Pt}(\mathrm{Me}-$ Duphos $)(\mathrm{Ph})]_{2}\left(\mu-\mathrm{CO}_{3}\right)$, glu220. $\mathrm{U}(\mathrm{eq})$ is defined as one third of the trace of the orthogonalized $\mathrm{U}^{\mathrm{ij}}$ tensor.

\begin{tabular}{|c|c|c|c|c|}
\hline & $\mathrm{x}$ & $\mathrm{y}$ & $\mathrm{z}$ & $\mathrm{U}(\mathrm{eq})$ \\
\hline $\operatorname{Pt}(1)$ & $3725(1)$ & $6815(1)$ & $7695(1)$ & $29(1)$ \\
\hline $\operatorname{Pt}(2)$ & 1261(1) & $4247(1)$ & $7258(1)$ & $31(1)$ \\
\hline $\operatorname{Pt}\left(1^{\prime}\right)$ & 1761(1) & $8748(1)$ & $9863(1)$ & $36(1)$ \\
\hline $\operatorname{Pt}\left(2^{\prime}\right)$ & $4461(1)$ & $11122(1)$ & $10236(1)$ & $36(1)$ \\
\hline $\mathrm{P}(1)$ & $3876(3)$ & $7126(2)$ & $7148(1)$ & $34(1)$ \\
\hline $\mathrm{P}\left(1^{\prime}\right)$ & $1514(2)$ & $8641(3)$ & $10420(1)$ & $37(1)$ \\
\hline $\mathrm{P}(2)$ & $4687(2)$ & $7733(2)$ & 7793(1) & $33(1)$ \\
\hline $\mathrm{P}\left(2^{\prime}\right)$ & $825(3)$ & 7813(3) & 9801(1) & $40(1)$ \\
\hline $\mathrm{P}(3)$ & $1268(3)$ & $3901(2)$ & 7801(1) & $37(1)$ \\
\hline $\mathrm{P}\left(3^{\prime}\right)$ & 4877(3) & 11033(3) & $9697(1)$ & $40(1)$ \\
\hline $\mathrm{P}(4)$ & $538(2)$ & $3144(2)$ & 7171(1) & $31(1)$ \\
\hline $\mathrm{P}\left(4^{\prime}\right)$ & $5562(3)$ & 11893(3) & 10319(1) & $39(1)$ \\
\hline $\mathrm{O}(1)$ & $2653(7)$ & $6152(7)$ & $7633(3)$ & $45(3)$ \\
\hline $\mathrm{O}\left(1^{\prime}\right)$ & $2481(10)$ & $9815(8)$ & 9913(4) & $69(4)$ \\
\hline $\mathrm{O}\left(2^{\prime}\right)$ & $3311(8)$ & $10628(7)$ & $10137(4)$ & $55(3)$ \\
\hline $\mathrm{O}(2)$ & $3139(8)$ & $5095(8)$ & $7348(3)$ & $55(3)$ \\
\hline $\mathrm{O}\left(3^{\prime}\right)$ & $3637(9)$ & $9320(9)$ & $10146(4)$ & $71(4)$ \\
\hline $\mathrm{O}(3)$ & $1772(7)$ & $5360(6)$ & $7385(3)$ & $39(3)$ \\
\hline$C(1)$ & $5277(15)$ & $6366(14)$ & 6811(7) & $77(7)$ \\
\hline $\mathrm{C}\left(1^{\prime}\right)$ & $1106(17)$ & $10312(12)$ & $10449(7)$ & $75(7)$ \\
\hline $\mathrm{C}(2)$ & $4298(14)$ & $6319(11)$ & $6867(5)$ & $58(5)$ \\
\hline$C\left(2^{\prime}\right)$ & $1295(15)$ & $9566(11)$ & $10665(5)$ & $57(5)$ \\
\hline$C\left(3^{\prime}\right)$ & $2132(17)$ & $9680(12)$ & $10863(6)$ & $70(7)$ \\
\hline$C(3)$ & $3690(20)$ & $6349(15)$ & $6570(5)$ & $87(9)$ \\
\hline$C\left(4^{\prime}\right)$ & $2344(17)$ & $8837(16)$ & 10993(5) & $75(7)$ \\
\hline $\mathrm{C}(4)$ & $2831(17)$ & $6574(14)$ & $6675(5)$ & $74(8)$ \\
\hline
\end{tabular}




\begin{tabular}{|c|c|c|c|c|}
\hline$C(5)$ & $2946(11)$ & $7307(11)$ & 6886(4) & $45(4)$ \\
\hline$C\left(5^{\prime}\right)$ & $2374(11)$ & $8251(12)$ & $10700(4)$ & $47(4)$ \\
\hline$C\left(6^{\prime}\right)$ & $2344(12)$ & $7378(13)$ & 10797(6) & $65(6)$ \\
\hline$C(6)$ & $2175(11)$ & $7556(12)$ & $7089(5)$ & $50(4)$ \\
\hline$C\left(7^{\prime}\right)$ & $598(11)$ & $8017(9)$ & 10473(3) & $36(4)$ \\
\hline$C(7)$ & $4533(10)$ & $8011(9)$ & $7126(4)$ & $33(3)$ \\
\hline $\mathrm{C}\left(8^{\prime}\right)$ & $163(12)$ & $7878(11)$ & $10788(4)$ & $45(4)$ \\
\hline$C(8)$ & $4716(13)$ & $8435(12)$ & $6826(5)$ & $57(5)$ \\
\hline$C\left(9^{\prime}\right)$ & $-502(11)$ & $7373(11)$ & $10795(5)$ & $50(4)$ \\
\hline$C(9)$ & $5197(12)$ & $9128(11)$ & $6834(5)$ & $51(5)$ \\
\hline $\mathrm{C}\left(10^{\prime}\right)$ & $-781(11)$ & 7005(9) & $10523(5)$ & $45(4)$ \\
\hline $\mathrm{C}(10)$ & $5532(13)$ & $9408(10)$ & $7115(4)$ & $49(4)$ \\
\hline $\mathrm{C}\left(11^{\prime}\right)$ & $-398(11)$ & $7142(10)$ & $10224(5)$ & $46(4)$ \\
\hline $\mathrm{C}(11)$ & $5395(10)$ & 8992(9) & $7422(4)$ & $40(4)$ \\
\hline$C\left(12^{\prime}\right)$ & $288(10)$ & $7625(9)$ & $10208(4)$ & $37(4)$ \\
\hline $\mathrm{C}(12)$ & $4887(11)$ & $8318(11)$ & $7421(5)$ & $48(4)$ \\
\hline $\mathrm{C}(13)$ & $4028(15)$ & $9175(13)$ & $8063(8)$ & $85(9)$ \\
\hline$C\left(13^{\prime}\right)$ & $1967(15)$ & $6541(14)$ & $9667(8)$ & $100(11)$ \\
\hline$C(14)$ & $4458(17)$ & $8445(13)$ & $8139(5)$ & $77(8)$ \\
\hline$C\left(14^{\prime}\right)$ & $1083(12)$ & $6763(10)$ & $9647(5)$ & $54(5)$ \\
\hline$C(15)$ & $5267(12)$ & $8518(12)$ & $8320(5)$ & $54(5)$ \\
\hline$C\left(15^{\prime}\right)$ & $638(18)$ & $6773(14)$ & $9299(5)$ & $78(8)$ \\
\hline$C(16)$ & $5749(14)$ & $7726(14)$ & $8302(6)$ & $71(7)$ \\
\hline$C\left(16^{\prime}\right)$ & $-160(20)$ & $7136(15)$ & $9324(6)$ & $84(8)$ \\
\hline $\mathrm{C}(17)$ & $5736(10)$ & $7481(12)$ & 7936(5) & $51(5)$ \\
\hline$C\left(17^{\prime}\right)$ & $-33(13)$ & $8007(14)$ & $9499(5)$ & $56(5)$ \\
\hline $\mathrm{C}(18)$ & $6004(12)$ & $6614(13)$ & 7854(6) & $64(6)$ \\
\hline$C\left(18^{\prime}\right)$ & $-730(30)$ & $8390(20)$ & $9588(9)$ & $150(20)$ \\
\hline$C(19)$ & $3582(9)$ & $6527(8)$ & 8193(3) & $28(3)$ \\
\hline$C\left(19^{\prime}\right)$ & $1887(11)$ & $8944(11)$ & $9365(3)$ & 41(4) \\
\hline$C\left(20^{\prime}\right)$ & $2214(12)$ & $8426(11)$ & $9126(5)$ & $46(4)$ \\
\hline
\end{tabular}




\begin{tabular}{|c|c|c|c|c|}
\hline$C(20)$ & $2793(11)$ & $6728(11)$ & 8334(4) & $44(4)$ \\
\hline $\mathrm{C}\left(21^{\prime}\right)$ & $2332(11)$ & $8657(12)$ & 8796(4) & $47(4)$ \\
\hline $\mathrm{C}(21)$ & $2620(12)$ & $6535(11)$ & $8658(4)$ & $46(4)$ \\
\hline $\mathrm{C}(22)$ & $3155(14)$ & $6100(11)$ & $8847(4)$ & $53(5)$ \\
\hline$C\left(22^{\prime}\right)$ & $2100(18)$ & $9404(12)$ & $8701(5)$ & $70(7)$ \\
\hline $\mathrm{C}(23)$ & $3939(12)$ & $5909(11)$ & $8710(4)$ & $47(4)$ \\
\hline$C\left(23^{\prime}\right)$ & $1644(16)$ & $9916(12)$ & 8911(5) & $66(7)$ \\
\hline $\mathrm{C}(24)$ & $4125(12)$ & 6143(9) & $8385(4)$ & $42(4)$ \\
\hline$C\left(24^{\prime}\right)$ & 1611(12) & $9704(11)$ & 9241(4) & $48(4)$ \\
\hline$C(25)$ & $-1198(11)$ & $3325(12)$ & $7154(5)$ & $58(5)$ \\
\hline$C\left(25^{\prime}\right)$ & $6447(16)$ & $10710(15)$ & $10702(6)$ & $77(7)$ \\
\hline$C(26)$ & $-458(11)$ & $3252(11)$ & $6937(5)$ & $47(4)$ \\
\hline$C\left(26^{\prime}\right)$ & $6378(13)$ & $11599(13)$ & $10626(5)$ & $58(5)$ \\
\hline$C\left(27^{\prime}\right)$ & $6202(19)$ & $12114(18)$ & 10922(6) & $92(9)$ \\
\hline$C(27)$ & $-474(11)$ & $2555(12)$ & $6692(5)$ & $49(4)$ \\
\hline$C\left(28^{\prime}\right)$ & $5931(14)$ & $12979(12)$ & $10778(5)$ & $58(5)$ \\
\hline $\mathrm{C}(28)$ & $428(12)$ & $2430(13)$ & $6570(4)$ & $53(5)$ \\
\hline$C(29)$ & $945(12)$ & $2347(10)$ & $6891(4)$ & $45(4)$ \\
\hline $\mathrm{C}\left(29^{\prime}\right)$ & $5380(13)$ & $12866(11)$ & $10522(5)$ & $54(5)$ \\
\hline $\mathrm{C}(30)$ & 1903(11) & $2351(12)$ & $6846(5)$ & $54(5)$ \\
\hline $\mathrm{C}\left(30^{\prime}\right)$ & $5271(16)$ & $13627(12)$ & $10314(8)$ & $86(8)$ \\
\hline $\mathrm{C}(31)$ & $676(9)$ & 2970(9) & $7855(4)$ & $31(3)$ \\
\hline $\mathrm{C}\left(31^{\prime}\right)$ & $6068(10)$ & $12072(11)$ & 9924(5) & $46(4)$ \\
\hline $\mathrm{C}\left(32^{\prime}\right)$ & $6807(11)$ & $12595(12)$ & $9906(4)$ & $48(4)$ \\
\hline $\mathrm{C}(32)$ & $315(9)$ & $2648(8)$ & 7564(3) & $28(3)$ \\
\hline $\mathrm{C}(33)$ & $-137(9)$ & 1959(9) & $7588(3)$ & $30(3)$ \\
\hline$C\left(33^{\prime}\right)$ & $7151(13)$ & $12734(12)$ & $9602(5)$ & $57(5)$ \\
\hline$C\left(34^{\prime}\right)$ & $6885(13)$ & $12327(14)$ & $9315(6)$ & $64(6)$ \\
\hline $\mathrm{C}(34)$ & $-271(11)$ & $1557(11)$ & 7884(4) & $43(4)$ \\
\hline$C\left(35^{\prime}\right)$ & $6134(12)$ & $11830(12)$ & $9338(5)$ & $56(5)$ \\
\hline $\mathrm{C}(35)$ & $71(10)$ & $1887(11)$ & $8168(4)$ & $40(4)$ \\
\hline
\end{tabular}




\begin{tabular}{|c|c|c|c|c|}
\hline$C\left(36^{\prime}\right)$ & $5754(11)$ & 11715(9) & $9647(4)$ & $42(4)$ \\
\hline$C(36)$ & $522(11)$ & $2569(10)$ & $8158(4)$ & $42(4)$ \\
\hline$C\left(37^{\prime}\right)$ & $3326(12)$ & $11646(14)$ & $9460(6)$ & $72(7)$ \\
\hline$C(37)$ & 2732(13) & $2938(17)$ & $7931(11)$ & $125(15)$ \\
\hline$C\left(38^{\prime}\right)$ & $4142(11)$ & $11247(12)$ & 9341(4) & $45(4)$ \\
\hline $\mathrm{C}(38)$ & $2322(12)$ & $3860(20)$ & $7983(5)$ & $87(10)$ \\
\hline $\mathrm{C}\left(39^{\prime}\right)$ & $4101(15)$ & $10383(17)$ & $9165(5)$ & $77(8)$ \\
\hline$C(39)$ & $2160(30)$ & $4240(30)$ & $8342(9)$ & $180(30)$ \\
\hline$C\left(40^{\prime}\right)$ & $4876(17)$ & $9987(13)$ & 9199(5) & $65(6)$ \\
\hline $\mathrm{C}(40)$ & $1800(40)$ & $4810(30)$ & $8296(8)$ & $210(40)$ \\
\hline $\mathrm{C}(41)$ & $870(20)$ & $4590(12)$ & $8138(5)$ & $84(9)$ \\
\hline$C\left(41^{\prime}\right)$ & $5167(12)$ & $10012(12)$ & 9554(4) & $47(4)$ \\
\hline$C\left(42^{\prime}\right)$ & $6132(17)$ & $9862(15)$ & $9610(6)$ & $79(7)$ \\
\hline$C(42)$ & $450(40)$ & $5228(16)$ & $8009(9)$ & $220(30)$ \\
\hline$C\left(43^{\prime}\right)$ & $4157(9)$ & $11090(11)$ & 10736(4) & $40(4)$ \\
\hline$C(43)$ & 1371(11) & $4526(9)$ & $6763(5)$ & $44(4)$ \\
\hline$C(44)$ & $2300(20)$ & $4669(16)$ & $6284(7)$ & $97(12)$ \\
\hline $\mathrm{C}\left(44^{\prime}\right)$ & $4258(14)$ & $10325(13)$ & $10889(5)$ & $60(5)$ \\
\hline $\mathrm{C}(45)$ & $1630(20)$ & $4948(16)$ & 6089(5) & $87(10)$ \\
\hline$C\left(45^{\prime}\right)$ & $4113(14)$ & $10266(18)$ & $11232(6)$ & $78(8)$ \\
\hline $\mathrm{C}(46)$ & $882(19)$ & $5006(14)$ & $6242(5)$ & $72(7)$ \\
\hline$C\left(46^{\prime}\right)$ & $3940(20)$ & $10960(17)$ & $11419(5)$ & 104(12) \\
\hline $\mathrm{C}(47)$ & $723(15)$ & $4827(14)$ & $6555(5)$ & $66(6)$ \\
\hline$C\left(47^{\prime}\right)$ & $3694(15)$ & $11635(17)$ & $11256(5)$ & $73(6)$ \\
\hline $\mathrm{C}(48)$ & $2177(16)$ & $4454(11)$ & $6617(5)$ & $63(6)$ \\
\hline$C\left(48^{\prime}\right)$ & $3870(10)$ & $11683(15)$ & $10929(4)$ & $55(5)$ \\
\hline $\mathrm{C}\left(49^{\prime}\right)$ & $3178(10)$ & $9878(11)$ & 10071(4) & $41(4)$ \\
\hline $\mathrm{C}(49)$ & $2521(10)$ & $5465(9)$ & $7448(4)$ & $33(3)$ \\
\hline
\end{tabular}


Table 3. Bond lengths $[\AA]$ and angles $[\mathrm{deg}]$ for $[\mathrm{Pt}(\mathrm{Me}-\mathrm{Duphos})(\mathrm{Ph})]_{2}\left(\mu-\mathrm{CO}_{3}\right)$, glu220.

\begin{tabular}{|c|c|c|c|}
\hline $\mathrm{Pt}(1)-\mathrm{O}(1)$ & $2.044(9)$ & $\mathrm{P}(3)-\mathrm{C}(38)$ & $1.834(18)$ \\
\hline $\operatorname{Pt}(1)-C(19)$ & $2.076(14)$ & $\mathrm{P}(3)-\mathrm{C}(41)$ & $1.89(2)$ \\
\hline $\mathrm{Pt}(1)-\mathrm{P}(2)$ & $2.194(4)$ & $\mathrm{P}\left(3^{\prime}\right)-\mathrm{C}\left(36^{\prime}\right)$ & $1.806(17)$ \\
\hline $\mathrm{Pt}(1)-\mathrm{P}(1)$ & $2.282(4)$ & $\mathrm{P}\left(3^{\prime}\right)-\mathrm{C}\left(41^{\prime}\right)$ & $1.845(19)$ \\
\hline $\mathrm{Pt}(2)-\mathrm{C}(43)$ & $2.057(19)$ & $\mathrm{P}\left(3^{\prime}\right)-\mathrm{C}\left(38^{\prime}\right)$ & $1.885(17)$ \\
\hline $\mathrm{Pt}(2)-\mathrm{O}(3)$ & $2.079(8)$ & $\mathrm{P}(4)-\mathrm{C}(32)$ & $1.821(15)$ \\
\hline $\mathrm{Pt}(2)-\mathrm{P}(4)$ & $2.186(4)$ & $\mathrm{P}(4)-\mathrm{C}(29)$ & $1.855(17)$ \\
\hline $\mathrm{Pt}(2)-\mathrm{P}(3)$ & $2.266(4)$ & $\mathrm{P}(4)-\mathrm{C}(26)$ & $1.856(17)$ \\
\hline $\operatorname{Pt}\left(1^{\prime}\right)-\mathrm{C}\left(19^{\prime}\right)$ & $2.045(14)$ & $\mathrm{P}\left(4^{\prime}\right)-\mathrm{C}\left(31^{\prime}\right)$ & $1.810(18)$ \\
\hline $\operatorname{Pt}\left(1^{\prime}\right)-\mathrm{O}\left(1^{\prime}\right)$ & $2.114(12)$ & $\mathrm{P}\left(4^{\prime}\right)-\mathrm{C}\left(29^{\prime}\right)$ & $1.829(18)$ \\
\hline $\operatorname{Pt}\left(1^{\prime}\right)-\mathrm{P}\left(2^{\prime}\right)$ & $2.163(4)$ & $\mathrm{P}\left(4^{\prime}\right)-\mathrm{C}\left(26^{\prime}\right)$ & $1.86(2)$ \\
\hline $\operatorname{Pt}\left(1^{\prime}\right)-\mathrm{P}\left(1^{\prime}\right)$ & $2.292(4)$ & $\mathrm{O}(1)-\mathrm{C}(49)$ & $1.374(18)$ \\
\hline $\operatorname{Pt}\left(2^{\prime}\right)-\mathrm{O}\left(2^{\prime}\right)$ & $2.044(13)$ & $\mathrm{O}\left(1^{\prime}\right)-\mathrm{C}\left(49^{\prime}\right)$ & $1.28(2)$ \\
\hline $\operatorname{Pt}\left(2^{\prime}\right)-\mathrm{C}\left(43^{\prime}\right)$ & $2.075(16)$ & $\mathrm{O}\left(2^{\prime}\right)-\mathrm{C}\left(49^{\prime}\right)$ & $1.29(2)$ \\
\hline $\operatorname{Pt}\left(2^{\prime}\right)-\mathrm{P}\left(4^{\prime}\right)$ & $2.194(4)$ & $\mathrm{O}(2)-\mathrm{C}(49)$ & $1.23(2)$ \\
\hline $\operatorname{Pt}\left(2^{\prime}\right)-\mathrm{P}\left(3^{\prime}\right)$ & $2.282(4)$ & $\mathrm{O}\left(3^{\prime}\right)-\mathrm{C}\left(49^{\prime}\right)$ & $1.22(2)$ \\
\hline $\mathrm{P}(1)-\mathrm{C}(7)$ & $1.802(14)$ & $\mathrm{O}(3)-\mathrm{C}(49)$ & $1.231(19)$ \\
\hline $\mathrm{P}(1)-\mathrm{C}(5)$ & $1.843(18)$ & $\mathrm{C}(1)-\mathrm{C}(2)$ & $1.58(3)$ \\
\hline $\mathrm{P}(1)-\mathrm{C}(2)$ & $1.875(17)$ & $\mathrm{C}\left(1^{\prime}\right)-\mathrm{C}\left(2^{\prime}\right)$ & $1.54(3)$ \\
\hline $\mathrm{P}\left(1^{\prime}\right)-\mathrm{C}\left(7^{\prime}\right)$ & $1.800(19)$ & $\mathrm{C}(2)-\mathrm{C}(3)$ & $1.55(4)$ \\
\hline $\mathrm{P}\left(1^{\prime}\right)-\mathrm{C}\left(2^{\prime}\right)$ & $1.855(19)$ & $\mathrm{C}\left(2^{\prime}\right)-\mathrm{C}\left(3^{\prime}\right)$ & $1.56(3)$ \\
\hline $\mathrm{P}\left(1^{\prime}\right)-\mathrm{C}\left(5^{\prime}\right)$ & $1.888(17)$ & $C\left(3^{\prime}\right)-C\left(4^{\prime}\right)$ & $1.53(3)$ \\
\hline $\mathrm{P}(2)-\mathrm{C}(17)$ & $1.815(17)$ & $C(3)-C(4)$ & $1.48(4)$ \\
\hline $\mathrm{P}(2)-\mathrm{C}(12)$ & $1.813(19)$ & $C\left(4^{\prime}\right)-C\left(5^{\prime}\right)$ & $1.53(3)$ \\
\hline $\mathrm{P}(2)-\mathrm{C}(14)$ & $1.863(19)$ & $C(4)-C(5)$ & $1.49(3)$ \\
\hline $\mathrm{P}\left(2^{\prime}\right)-\mathrm{C}\left(17^{\prime}\right)$ & $1.86(2)$ & $C(5)-C(6)$ & $1.53(3)$ \\
\hline $\mathrm{P}\left(2^{\prime}\right)-\mathrm{C}\left(12^{\prime}\right)$ & $1.881(14)$ & $\mathrm{C}\left(5^{\prime}\right)-\mathrm{C}\left(6^{\prime}\right)$ & $1.50(3)$ \\
\hline $\mathrm{P}\left(2^{\prime}\right)-\mathrm{C}\left(14^{\prime}\right)$ & $1.890(17)$ & $C\left(7^{\prime}\right)-C\left(12^{\prime}\right)$ & $1.34(2)$ \\
\hline $\mathrm{P}(3)-\mathrm{C}(31)$ & $1.818(15)$ & $\mathrm{C}\left(7^{\prime}\right)-\mathrm{C}\left(8^{\prime}\right)$ & $1.47(2)$ \\
\hline
\end{tabular}




\begin{tabular}{|c|c|c|c|}
\hline$C(7)-C(12)$ & $1.41(2)$ & $\mathrm{C}\left(23^{\prime}\right)-\mathrm{C}\left(24^{\prime}\right)$ & $1.38(2)$ \\
\hline$C(7)-C(8)$ & $1.43(2)$ & $C(25)-C(26)$ & $1.47(3)$ \\
\hline $\mathrm{C}\left(8^{\prime}\right)-\mathrm{C}\left(9^{\prime}\right)$ & $1.35(3)$ & $\mathrm{C}\left(25^{\prime}\right)-\mathrm{C}\left(26^{\prime}\right)$ & $1.51(3)$ \\
\hline $\mathrm{C}(8)-\mathrm{C}(9)$ & $1.38(3)$ & $C(26)-C(27)$ & $1.52(2)$ \\
\hline$C\left(9^{\prime}\right)-C\left(10^{\prime}\right)$ & $1.33(3)$ & $C\left(26^{\prime}\right)-C\left(27^{\prime}\right)$ & $1.49(3)$ \\
\hline$C(9)-C(10)$ & $1.34(3)$ & $\mathrm{C}\left(27^{\prime}\right)-\mathrm{C}\left(28^{\prime}\right)$ & $1.60(4)$ \\
\hline $\mathrm{C}\left(10^{\prime}\right)-\mathrm{C}\left(11^{\prime}\right)$ & $1.37(3)$ & $C(27)-C(28)$ & $1.53(3)$ \\
\hline$C(10)-C(11)$ & $1.43(2)$ & $\mathrm{C}\left(28^{\prime}\right)-\mathrm{C}\left(29^{\prime}\right)$ & $1.37(3)$ \\
\hline $\mathrm{C}\left(11^{\prime}\right)-\mathrm{C}\left(12^{\prime}\right)$ & $1.35(2)$ & $C(28)-C(29)$ & $1.54(2)$ \\
\hline$C(11)-C(12)$ & $1.38(2)$ & $C(29)-C(30)$ & $1.54(3)$ \\
\hline$C(13)-C(14)$ & $1.42(4)$ & $\mathrm{C}\left(29^{\prime}\right)-\mathrm{C}\left(30^{\prime}\right)$ & $1.52(3)$ \\
\hline $\mathrm{C}\left(13^{\prime}\right)-\mathrm{C}\left(14^{\prime}\right)$ & $1.46(3)$ & $\mathrm{C}(31)-\mathrm{C}(36)$ & $1.41(2)$ \\
\hline$C(14)-C(15)$ & $1.49(3)$ & $\mathrm{C}(31)-\mathrm{C}(32)$ & $1.41(2)$ \\
\hline $\mathrm{C}\left(14^{\prime}\right)-\mathrm{C}\left(15^{\prime}\right)$ & $1.57(3)$ & $\mathrm{C}\left(31^{\prime}\right)-\mathrm{C}\left(36^{\prime}\right)$ & $1.36(2)$ \\
\hline$C(15)-C(16)$ & $1.52(3)$ & $\mathrm{C}\left(31^{\prime}\right)-\mathrm{C}\left(32^{\prime}\right)$ & $1.46(2)$ \\
\hline$C\left(15^{\prime}\right)-C\left(16^{\prime}\right)$ & $1.41(4)$ & $\mathrm{C}\left(32^{\prime}\right)-\mathrm{C}\left(33^{\prime}\right)$ & $1.36(2)$ \\
\hline$C(16)-C(17)$ & $1.53(3)$ & $\mathrm{C}(32)-\mathrm{C}(33)$ & $1.35(2)$ \\
\hline$C\left(16^{\prime}\right)-C\left(17^{\prime}\right)$ & $1.62(3)$ & $C(33)-C(34)$ & $1.39(2)$ \\
\hline$C(17)-C(18)$ & $1.53(3)$ & $\mathrm{C}\left(33^{\prime}\right)-\mathrm{C}\left(34^{\prime}\right)$ & $1.41(3)$ \\
\hline $\mathrm{C}\left(17^{\prime}\right)-\mathrm{C}\left(18^{\prime}\right)$ & $1.32(5)$ & $\mathrm{C}\left(34^{\prime}\right)-\mathrm{C}\left(35^{\prime}\right)$ & $1.45(3)$ \\
\hline$C(19)-C(24)$ & $1.32(2)$ & $\mathrm{C}(34)-\mathrm{C}(35)$ & $1.38(2)$ \\
\hline$C(19)-C(20)$ & $1.42(2)$ & $\mathrm{C}\left(35^{\prime}\right)-\mathrm{C}\left(36^{\prime}\right)$ & $1.40(2)$ \\
\hline $\mathrm{C}\left(19^{\prime}\right)-\mathrm{C}\left(20^{\prime}\right)$ & $1.39(2)$ & $C(35)-C(36)$ & $1.34(2)$ \\
\hline $\mathrm{C}\left(19^{\prime}\right)-\mathrm{C}\left(24^{\prime}\right)$ & $1.42(2)$ & $\mathrm{C}\left(37^{\prime}\right)-\mathrm{C}\left(38^{\prime}\right)$ & $1.53(3)$ \\
\hline $\mathrm{C}\left(20^{\prime}\right)-\mathrm{C}\left(21^{\prime}\right)$ & $1.40(2)$ & $\mathrm{C}(37)-\mathrm{C}(38)$ & $1.68(4)$ \\
\hline$C(20)-C(21)$ & $1.37(2)$ & $\mathrm{C}\left(38^{\prime}\right)-\mathrm{C}\left(39^{\prime}\right)$ & $1.60(3)$ \\
\hline $\mathrm{C}\left(21^{\prime}\right)-\mathrm{C}\left(22^{\prime}\right)$ & $1.35(3)$ & $\mathrm{C}(38)-\mathrm{C}(39)$ & $1.60(6)$ \\
\hline$C(21)-C(22)$ & $1.35(3)$ & $\mathrm{C}\left(39^{\prime}\right)-\mathrm{C}\left(40^{\prime}\right)$ & $1.40(3)$ \\
\hline$C(22)-C(23)$ & $1.40(3)$ & $\mathrm{C}(39)-\mathrm{C}(40)$ & $1.12(9)$ \\
\hline $\mathrm{C}\left(22^{\prime}\right)-\mathrm{C}\left(23^{\prime}\right)$ & $1.40(3)$ & $\mathrm{C}\left(40^{\prime}\right)-\mathrm{C}\left(41^{\prime}\right)$ & $1.51(3)$ \\
\hline$C(23)-C(24)$ & $1.40(2)$ & $\mathrm{C}(40)-\mathrm{C}(41)$ & $1.66(6)$ \\
\hline
\end{tabular}




\begin{tabular}{|c|c|c|c|}
\hline$C(41)-C(42)$ & $1.35(5)$ & $\mathrm{O}\left(1^{\prime}\right)-\mathrm{Pt}\left(1^{\prime}\right)-\mathrm{P}\left(1^{\prime}\right)$ & $93.6(4)$ \\
\hline $\mathrm{C}\left(41^{\prime}\right)-\mathrm{C}\left(42^{\prime}\right)$ & $1.57(3)$ & $\mathrm{P}\left(2^{\prime}\right)-\mathrm{Pt}\left(1^{\prime}\right)-\mathrm{P}\left(1^{\prime}\right)$ & $86.56(16)$ \\
\hline $\mathrm{C}\left(43^{\prime}\right)-\mathrm{C}\left(48^{\prime}\right)$ & $1.33(3)$ & $\mathrm{O}\left(2^{\prime}\right)-\mathrm{Pt}\left(2^{\prime}\right)-\mathrm{C}\left(43^{\prime}\right)$ & $88.3(6)$ \\
\hline $\mathrm{C}\left(43^{\prime}\right)-\mathrm{C}\left(44^{\prime}\right)$ & $1.42(3)$ & $\mathrm{O}\left(2^{\prime}\right)-\mathrm{Pt}\left(2^{\prime}\right)-\mathrm{P}\left(4^{\prime}\right)$ & $167.9(4)$ \\
\hline$C(43)-C(48)$ & $1.42(3)$ & $\mathrm{C}\left(43^{\prime}\right)-\mathrm{Pt}\left(2^{\prime}\right)-\mathrm{P}\left(4^{\prime}\right)$ & $93.0(5)$ \\
\hline$C(43)-C(47)$ & $1.42(3)$ & $\mathrm{O}\left(2^{\prime}\right)-\mathrm{Pt}\left(2^{\prime}\right)-\mathrm{P}\left(3^{\prime}\right)$ & $92.8(4)$ \\
\hline$C(44)-C(48)$ & $1.40(3)$ & $\mathrm{C}\left(43^{\prime}\right)-\mathrm{Pt}\left(2^{\prime}\right)-\mathrm{P}\left(3^{\prime}\right)$ & $173.9(5)$ \\
\hline$C(44)-C(45)$ & $1.40(4)$ & $\mathrm{P}\left(4^{\prime}\right)-\mathrm{Pt}\left(2^{\prime}\right)-\mathrm{P}\left(3^{\prime}\right)$ & $87.18(15)$ \\
\hline$C\left(44^{\prime}\right)-C\left(45^{\prime}\right)$ & $1.41(3)$ & $\mathrm{C}(7)-\mathrm{P}(1)-\mathrm{C}(5)$ & $107.8(8)$ \\
\hline$C(45)-C(46)$ & $1.34(4)$ & $\mathrm{C}(7)-\mathrm{P}(1)-\mathrm{C}(2)$ & $109.9(8)$ \\
\hline$C\left(45^{\prime}\right)-C\left(46^{\prime}\right)$ & $1.40(4)$ & $\mathrm{C}(5)-\mathrm{P}(1)-\mathrm{C}(2)$ & $93.3(10)$ \\
\hline$C(46)-C(47)$ & $1.32(3)$ & $\mathrm{C}(7)-\mathrm{P}(1)-\mathrm{Pt}(1)$ & $107.0(5)$ \\
\hline $\mathrm{C}\left(46^{\prime}\right)-\mathrm{C}\left(47^{\prime}\right)$ & $1.35(4)$ & $\mathrm{C}(5)-\mathrm{P}(1)-\mathrm{Pt}(1)$ & $120.5(6)$ \\
\hline \multirow[t]{2}{*}{$\mathrm{C}\left(47^{\prime}\right)-\mathrm{C}\left(48^{\prime}\right)$} & $1.35(3)$ & $\mathrm{C}(2)-\mathrm{P}(1)-\mathrm{Pt}(1)$ & $117.6(7)$ \\
\hline & & $\mathrm{C}\left(7^{\prime}\right)-\mathrm{P}\left(1^{\prime}\right)-\mathrm{C}\left(2^{\prime}\right)$ & $105.0(9)$ \\
\hline $\mathrm{O}(1)-\mathrm{Pt}(1)-\mathrm{C}(19)$ & $84.6(5)$ & $\mathrm{C}\left(7^{\prime}\right)-\mathrm{P}\left(1^{\prime}\right)-\mathrm{C}\left(5^{\prime}\right)$ & $108.7(8)$ \\
\hline $\mathrm{O}(1)-\mathrm{Pt}(1)-\mathrm{P}(2)$ & $167.7(4)$ & $\mathrm{C}\left(2^{\prime}\right)-\mathrm{P}\left(1^{\prime}\right)-\mathrm{C}\left(5^{\prime}\right)$ & $95.7(10)$ \\
\hline $\mathrm{C}(19)-\mathrm{Pt}(1)-\mathrm{P}(2)$ & $93.6(4)$ & $\mathrm{C}\left(7^{\prime}\right)-\mathrm{P}\left(1^{\prime}\right)-\mathrm{Pt}\left(1^{\prime}\right)$ & $107.4(5)$ \\
\hline $\mathrm{O}(1)-\mathrm{Pt}(1)-\mathrm{P}(1)$ & $95.1(3)$ & $\mathrm{C}\left(2^{\prime}\right)-\mathrm{P}\left(1^{\prime}\right)-\mathrm{Pt}\left(1^{\prime}\right)$ & $119.5(7)$ \\
\hline $\mathrm{C}(19)-\mathrm{Pt}(1)-\mathrm{P}(1)$ & $179.7(4)$ & $\mathrm{C}\left(5^{\prime}\right)-\mathrm{P}\left(1^{\prime}\right)-\mathrm{Pt}\left(1^{\prime}\right)$ & $119.3(6)$ \\
\hline $\mathrm{P}(2)-\mathrm{Pt}(1)-\mathrm{P}(1)$ & $86.77(14)$ & $\mathrm{C}(17)-\mathrm{P}(2)-\mathrm{C}(12)$ & $103.0(9)$ \\
\hline $\mathrm{C}(43)-\mathrm{Pt}(2)-\mathrm{O}(3)$ & $90.5(5)$ & $C(17)-P(2)-C(14)$ & $95.0(12)$ \\
\hline $\mathrm{C}(43)-\mathrm{Pt}(2)-\mathrm{P}(4)$ & $94.5(4)$ & $C(12)-P(2)-C(14)$ & $108.5(10)$ \\
\hline $\mathrm{O}(3)-\mathrm{Pt}(2)-\mathrm{P}(4)$ & $170.3(3)$ & $\mathrm{C}(17)-\mathrm{P}(2)-\mathrm{Pt}(1)$ & $122.7(6)$ \\
\hline $\mathrm{C}(43)-\mathrm{Pt}(2)-\mathrm{P}(3)$ & $174.6(5)$ & $\mathrm{C}(12)-\mathrm{P}(2)-\mathrm{Pt}(1)$ & $110.1(6)$ \\
\hline $\mathrm{O}(3)-\mathrm{Pt}(2)-\mathrm{P}(3)$ & $89.0(3)$ & $\mathrm{C}(14)-\mathrm{P}(2)-\mathrm{Pt}(1)$ & $115.8(7)$ \\
\hline $\mathrm{P}(4)-\mathrm{Pt}(2)-\mathrm{P}(3)$ & $86.86(14)$ & $\mathrm{C}\left(17^{\prime}\right)-\mathrm{P}\left(2^{\prime}\right)-\mathrm{C}\left(12^{\prime}\right)$ & $105.5(9)$ \\
\hline$C\left(19^{\prime}\right)-\operatorname{Pt}\left(1^{\prime}\right)-\mathrm{O}\left(1^{\prime}\right)$ & $84.8(6)$ & $\mathrm{C}\left(17^{\prime}\right)-\mathrm{P}\left(2^{\prime}\right)-\mathrm{C}\left(14^{\prime}\right)$ & $95.9(11)$ \\
\hline $\mathrm{C}\left(19^{\prime}\right)-\mathrm{Pt}\left(1^{\prime}\right)-\mathrm{P}\left(2^{\prime}\right)$ & $93.9(5)$ & $\mathrm{C}\left(12^{\prime}\right)-\mathrm{P}\left(2^{\prime}\right)-\mathrm{C}\left(14^{\prime}\right)$ & $103.6(8)$ \\
\hline $\mathrm{O}\left(1^{\prime}\right)-\mathrm{Pt}\left(1^{\prime}\right)-\mathrm{P}\left(2^{\prime}\right)$ & $169.1(5)$ & $\mathrm{C}\left(17^{\prime}\right)-\mathrm{P}\left(2^{\prime}\right)-\mathrm{Pt}\left(1^{\prime}\right)$ & $117.3(7)$ \\
\hline $\mathrm{C}\left(19^{\prime}\right)-\mathrm{Pt}\left(1^{\prime}\right)-\mathrm{P}\left(1^{\prime}\right)$ & $173.8(5)$ & $\mathrm{C}\left(12^{\prime}\right)-\mathrm{P}\left(2^{\prime}\right)-\mathrm{Pt}\left(1^{\prime}\right)$ & $109.3(6)$ \\
\hline
\end{tabular}




\begin{tabular}{|c|c|c|c|}
\hline C(14')-P(2')-Pt(1') & $123.1(6)$ & $\mathrm{C}(1)-\mathrm{C}(2)-\mathrm{P}(1)$ & $114.0(14)$ \\
\hline $\mathrm{C}(31)-\mathrm{P}(3)-\mathrm{C}(38)$ & $113.3(11)$ & $C\left(1^{\prime}\right)-C\left(2^{\prime}\right)-C\left(3^{\prime}\right)$ & $111.0(18)$ \\
\hline $\mathrm{C}(31)-\mathrm{P}(3)-\mathrm{C}(41)$ & $104.4(8)$ & $\mathrm{C}\left(1^{\prime}\right)-\mathrm{C}\left(2^{\prime}\right)-\mathrm{P}\left(1^{\prime}\right)$ & $113.3(14)$ \\
\hline $\mathrm{C}(38)-\mathrm{P}(3)-\mathrm{C}(41)$ & $92.3(14)$ & $\mathrm{C}\left(3^{\prime}\right)-\mathrm{C}\left(2^{\prime}\right)-\mathrm{P}\left(1^{\prime}\right)$ & $102.2(14)$ \\
\hline $\mathrm{C}(31)-\mathrm{P}(3)-\mathrm{Pt}(2)$ & $109.2(5)$ & $\mathrm{C}\left(4^{\prime}\right)-\mathrm{C}\left(3^{\prime}\right)-\mathrm{C}\left(2^{\prime}\right)$ & $104.7(17)$ \\
\hline C(38)-P(3)-Pt(2) & $113.6(7)$ & $\mathrm{C}(4)-\mathrm{C}(3)-\mathrm{C}(2)$ & $111.5(16)$ \\
\hline $\mathrm{C}(41)-\mathrm{P}(3)-\mathrm{Pt}(2)$ & $123.0(8)$ & $C\left(3^{\prime}\right)-C\left(4^{\prime}\right)-C\left(5^{\prime}\right)$ & $108.6(16)$ \\
\hline $\mathrm{C}\left(36^{\prime}\right)-\mathrm{P}\left(3^{\prime}\right)-\mathrm{C}\left(41^{\prime}\right)$ & $110.1(8)$ & $C(3)-C(4)-C(5)$ & $104.8(18)$ \\
\hline $\mathrm{C}\left(36^{\prime}\right)-\mathrm{P}\left(3^{\prime}\right)-\mathrm{C}\left(38^{\prime}\right)$ & $106.2(8)$ & $\mathrm{C}(4)-\mathrm{C}(5)-\mathrm{C}(6)$ & $115.2(17)$ \\
\hline C(41')-P(3')-C(38') & $95.1(8)$ & $\mathrm{C}(4)-\mathrm{C}(5)-\mathrm{P}(1)$ & $107.1(15)$ \\
\hline $\mathrm{C}\left(36^{\prime}\right)-\mathrm{P}\left(3^{\prime}\right)-\mathrm{Pt}\left(2^{\prime}\right)$ & $106.9(5)$ & $\mathrm{C}(6)-\mathrm{C}(5)-\mathrm{P}(1)$ & $112.4(12)$ \\
\hline $\mathrm{C}\left(41^{\prime}\right)-\mathrm{P}\left(3^{\prime}\right)-\mathrm{Pt}\left(2^{\prime}\right)$ & $115.4(6)$ & $\mathrm{C}\left(6^{\prime}\right)-\mathrm{C}\left(5^{\prime}\right)-\mathrm{C}\left(4^{\prime}\right)$ & $114.0(18)$ \\
\hline $\mathrm{C}\left(38^{\prime}\right)-\mathrm{P}\left(3^{\prime}\right)-\mathrm{Pt}\left(2^{\prime}\right)$ & $122.3(6)$ & $\mathrm{C}\left(6^{\prime}\right)-\mathrm{C}\left(5^{\prime}\right)-\mathrm{P}\left(1^{\prime}\right)$ & $117.6(14)$ \\
\hline $\mathrm{C}(32)-\mathrm{P}(4)-\mathrm{C}(29)$ & $106.2(7)$ & $\mathrm{C}\left(4^{\prime}\right)-\mathrm{C}\left(5^{\prime}\right)-\mathrm{P}\left(1^{\prime}\right)$ & $103.0(15)$ \\
\hline $\mathrm{C}(32)-\mathrm{P}(4)-\mathrm{C}(26)$ & $108.8(8)$ & $\mathrm{C}\left(12^{\prime}\right)-\mathrm{C}\left(7^{\prime}\right)-\mathrm{C}\left(8^{\prime}\right)$ & $116.2(16)$ \\
\hline C(29)-P(4)-C(26) & $93.1(8)$ & $\mathrm{C}\left(12^{\prime}\right)-\mathrm{C}\left(7^{\prime}\right)-\mathrm{P}\left(1^{\prime}\right)$ & $118.8(11)$ \\
\hline C(32)-P(4)-Pt(2) & $109.9(4)$ & $\mathrm{C}\left(8^{\prime}\right)-\mathrm{C}\left(7^{\prime}\right)-\mathrm{P}\left(1^{\prime}\right)$ & $125.0(13)$ \\
\hline C(29)-P(4)-Pt(2) & $120.5(6)$ & $\mathrm{C}(12)-\mathrm{C}(7)-\mathrm{C}(8)$ & $117.2(15)$ \\
\hline $\mathrm{C}(26)-\mathrm{P}(4)-\mathrm{Pt}(2)$ & $116.8(6)$ & $\mathrm{C}(12)-\mathrm{C}(7)-\mathrm{P}(1)$ & $118.8(11)$ \\
\hline $\mathrm{C}\left(31^{\prime}\right)-\mathrm{P}\left(4^{\prime}\right)-\mathrm{C}\left(29^{\prime}\right)$ & $108.8(9)$ & $\mathrm{C}(8)-\mathrm{C}(7)-\mathrm{P}(1)$ & $124.0(13)$ \\
\hline $\mathrm{C}\left(31^{\prime}\right)-\mathrm{P}\left(4^{\prime}\right)-\mathrm{C}\left(26^{\prime}\right)$ & $108.6(9)$ & $\mathrm{C}\left(9^{\prime}\right)-\mathrm{C}\left(8^{\prime}\right)-\mathrm{C}\left(7^{\prime}\right)$ & $118.9(16)$ \\
\hline $\mathrm{C}\left(29^{\prime}\right)-\mathrm{P}\left(4^{\prime}\right)-\mathrm{C}\left(26^{\prime}\right)$ & $92.4(10)$ & $\mathrm{C}(9)-\mathrm{C}(8)-\mathrm{C}(7)$ & $120.0(18)$ \\
\hline C(31')-P(4')-Pt(2') & $108.4(6)$ & $\mathrm{C}\left(10^{\prime}\right)-\mathrm{C}\left(9^{\prime}\right)-\mathrm{C}\left(8^{\prime}\right)$ & $121.9(18)$ \\
\hline $\mathrm{C}\left(29^{\prime}\right)-\mathrm{P}\left(4^{\prime}\right)-\operatorname{Pt}\left(2^{\prime}\right)$ & $117.0(7)$ & $\mathrm{C}(10)-\mathrm{C}(9)-\mathrm{C}(8)$ & $122.0(16)$ \\
\hline $\mathrm{C}\left(26^{\prime}\right)-\mathrm{P}\left(4^{\prime}\right)-\mathrm{Pt}\left(2^{\prime}\right)$ & $120.5(7)$ & $\mathrm{C}\left(9^{\prime}\right)-\mathrm{C}\left(10^{\prime}\right)-\mathrm{C}\left(11^{\prime}\right)$ & $120.2(17)$ \\
\hline C(49)-O(1)-Pt(1) & $129.7(10)$ & $\mathrm{C}(9)-\mathrm{C}(10)-\mathrm{C}(11)$ & $120.4(15)$ \\
\hline $\mathrm{C}\left(49^{\prime}\right)-\mathrm{O}\left(1^{\prime}\right)-\mathrm{Pt}\left(1^{\prime}\right)$ & $125.8(13)$ & $\mathrm{C}\left(12^{\prime}\right)-\mathrm{C}\left(11^{\prime}\right)-\mathrm{C}\left(10^{\prime}\right)$ & $119.7(17)$ \\
\hline $\mathrm{C}\left(49^{\prime}\right)-\mathrm{O}\left(2^{\prime}\right)-\mathrm{Pt}\left(2^{\prime}\right)$ & $125.0(11)$ & $\mathrm{C}(12)-\mathrm{C}(11)-\mathrm{C}(10)$ & $118.5(16)$ \\
\hline $\mathrm{C}(49)-\mathrm{O}(3)-\mathrm{Pt}(2)$ & $123.7(10)$ & $\mathrm{C}\left(7^{\prime}\right)-\mathrm{C}\left(12^{\prime}\right)-\mathrm{C}\left(11^{\prime}\right)$ & $123.0(15)$ \\
\hline$C(3)-C(2)-C(1)$ & $120.5(18)$ & $\mathrm{C}\left(7^{\prime}\right)-\mathrm{C}\left(12^{\prime}\right)-\mathrm{P}\left(2^{\prime}\right)$ & $116.7(12)$ \\
\hline $\mathrm{C}(3)-\mathrm{C}(2)-\mathrm{P}(1)$ & $102.8(15)$ & $\mathrm{C}\left(11^{\prime}\right)-\mathrm{C}\left(12^{\prime}\right)-\mathrm{P}\left(2^{\prime}\right)$ & $120.3(13)$ \\
\hline
\end{tabular}




\begin{tabular}{|c|c|c|c|}
\hline $\mathrm{C}(11)-\mathrm{C}(12)-\mathrm{C}(7)$ & $121.8(16)$ & $\mathrm{C}(22)-\mathrm{C}(23)-\mathrm{C}(24)$ & $119.9(17)$ \\
\hline $\mathrm{C}(11)-\mathrm{C}(12)-\mathrm{P}(2)$ & $122.2(15)$ & $\mathrm{C}\left(24^{\prime}\right)-\mathrm{C}\left(23^{\prime}\right)-\mathrm{C}\left(22^{\prime}\right)$ & $116.8(19)$ \\
\hline $\mathrm{C}(7)-\mathrm{C}(12)-\mathrm{P}(2)$ & $115.9(12)$ & $\mathrm{C}(19)-\mathrm{C}(24)-\mathrm{C}(23)$ & $122.9(18)$ \\
\hline $\mathrm{C}(13)-\mathrm{C}(14)-\mathrm{C}(15)$ & 117.1(17) & $\mathrm{C}\left(23^{\prime}\right)-\mathrm{C}\left(24^{\prime}\right)-\mathrm{C}\left(19^{\prime}\right)$ & $123.6(16)$ \\
\hline $\mathrm{C}(13)-\mathrm{C}(14)-\mathrm{P}(2)$ & $118.0(18)$ & $\mathrm{C}(25)-\mathrm{C}(26)-\mathrm{C}(27)$ & $115.9(16)$ \\
\hline $\mathrm{C}(15)-\mathrm{C}(14)-\mathrm{P}(2)$ & $104.5(17)$ & $\mathrm{C}(25)-\mathrm{C}(26)-\mathrm{P}(4)$ & $112.8(13)$ \\
\hline $\mathrm{C}\left(13^{\prime}\right)-\mathrm{C}\left(14^{\prime}\right)-\mathrm{C}\left(15^{\prime}\right)$ & $119(2)$ & $\mathrm{C}(27)-\mathrm{C}(26)-\mathrm{P}(4)$ & $105.9(11)$ \\
\hline $\mathrm{C}\left(13^{\prime}\right)-\mathrm{C}\left(14^{\prime}\right)-\mathrm{P}\left(2^{\prime}\right)$ & $115.0(15)$ & $\mathrm{C}\left(27^{\prime}\right)-\mathrm{C}\left(26^{\prime}\right)-\mathrm{C}\left(25^{\prime}\right)$ & $114(2)$ \\
\hline $\mathrm{C}\left(15^{\prime}\right)-\mathrm{C}\left(14^{\prime}\right)-\mathrm{P}\left(2^{\prime}\right)$ & $100.7(14)$ & $\mathrm{C}\left(27^{\prime}\right)-\mathrm{C}\left(26^{\prime}\right)-\mathrm{P}\left(4^{\prime}\right)$ & $104.6(16)$ \\
\hline $\mathrm{C}(14)-\mathrm{C}(15)-\mathrm{C}(16)$ & $110.2(16)$ & $\mathrm{C}\left(25^{\prime}\right)-\mathrm{C}\left(26^{\prime}\right)-\mathrm{P}\left(4^{\prime}\right)$ & $116.2(16)$ \\
\hline $\mathrm{C}\left(16^{\prime}\right)-\mathrm{C}\left(15^{\prime}\right)-\mathrm{C}\left(14^{\prime}\right)$ & $110.3(17)$ & $\mathrm{C}\left(26^{\prime}\right)-\mathrm{C}\left(27^{\prime}\right)-\mathrm{C}\left(28^{\prime}\right)$ & $105.7(18)$ \\
\hline $\mathrm{C}(15)-\mathrm{C}(16)-\mathrm{C}(17)$ & $105.4(17)$ & $\mathrm{C}(26)-\mathrm{C}(27)-\mathrm{C}(28)$ & $107.2(15)$ \\
\hline $\mathrm{C}\left(15^{\prime}\right)-\mathrm{C}\left(16^{\prime}\right)-\mathrm{C}\left(17^{\prime}\right)$ & $107(2)$ & $\mathrm{C}\left(29^{\prime}\right)-\mathrm{C}\left(28^{\prime}\right)-\mathrm{C}\left(27^{\prime}\right)$ & $108.9(16)$ \\
\hline $\mathrm{C}(16)-\mathrm{C}(17)-\mathrm{C}(18)$ & $116.9(18)$ & $\mathrm{C}(27)-\mathrm{C}(28)-\mathrm{C}(29)$ & $104.1(15)$ \\
\hline $\mathrm{C}(16)-\mathrm{C}(17)-\mathrm{P}(2)$ & $105.0(14)$ & $\mathrm{C}(30)-\mathrm{C}(29)-\mathrm{C}(28)$ & $115.5(17)$ \\
\hline C(18)-C(17)-P(2) & $113.7(13)$ & $\mathrm{C}(30)-\mathrm{C}(29)-\mathrm{P}(4)$ & $114.6(12)$ \\
\hline $\mathrm{C}\left(18^{\prime}\right)-\mathrm{C}\left(17^{\prime}\right)-\mathrm{C}\left(16^{\prime}\right)$ & $116(2)$ & $\mathrm{C}(28)-\mathrm{C}(29)-\mathrm{P}(4)$ & $105.3(13)$ \\
\hline $\mathrm{C}\left(18^{\prime}\right)-\mathrm{C}\left(17^{\prime}\right)-\mathrm{P}\left(2^{\prime}\right)$ & $121(2)$ & $\mathrm{C}\left(28^{\prime}\right)-\mathrm{C}\left(29^{\prime}\right)-\mathrm{C}\left(30^{\prime}\right)$ & $112.2(18)$ \\
\hline $\mathrm{C}\left(16^{\prime}\right)-\mathrm{C}\left(17^{\prime}\right)-\mathrm{P}\left(2^{\prime}\right)$ & $103.1(17)$ & $\mathrm{C}\left(28^{\prime}\right)-\mathrm{C}\left(29^{\prime}\right)-\mathrm{P}\left(4^{\prime}\right)$ & $110.9(16)$ \\
\hline$C(24)-C(19)-C(20)$ & $117.0(14)$ & $\mathrm{C}\left(30^{\prime}\right)-\mathrm{C}\left(29^{\prime}\right)-\mathrm{P}\left(4^{\prime}\right)$ & $119.9(16)$ \\
\hline$C(24)-C(19)-\operatorname{Pt}(1)$ & $127.2(12)$ & $C(36)-C(31)-C(32)$ & $118.1(14)$ \\
\hline$C(20)-C(19)-\operatorname{Pt}(1)$ & $115.6(11)$ & $\mathrm{C}(36)-\mathrm{C}(31)-\mathrm{P}(3)$ & $126.4(12)$ \\
\hline $\mathrm{C}\left(20^{\prime}\right)-\mathrm{C}\left(19^{\prime}\right)-\mathrm{C}\left(24^{\prime}\right)$ & $114.5(14)$ & $\mathrm{C}(32)-\mathrm{C}(31)-\mathrm{P}(3)$ & $115.4(10)$ \\
\hline $\mathrm{C}\left(20^{\prime}\right)-\mathrm{C}\left(19^{\prime}\right)-\operatorname{Pt}\left(1^{\prime}\right)$ & $128.4(14)$ & $\mathrm{C}\left(36^{\prime}\right)-\mathrm{C}\left(31^{\prime}\right)-\mathrm{C}\left(32^{\prime}\right)$ & $120.6(16)$ \\
\hline $\mathrm{C}\left(24^{\prime}\right)-\mathrm{C}\left(19^{\prime}\right)-\operatorname{Pt}\left(1^{\prime}\right)$ & $117.1(12)$ & $\mathrm{C}\left(36^{\prime}\right)-\mathrm{C}\left(31^{\prime}\right)-\mathrm{P}\left(4^{\prime}\right)$ & $119.4(11)$ \\
\hline $\mathrm{C}\left(19^{\prime}\right)-\mathrm{C}\left(20^{\prime}\right)-\mathrm{C}\left(21^{\prime}\right)$ & $122.9(17)$ & $\mathrm{C}\left(32^{\prime}\right)-\mathrm{C}\left(31^{\prime}\right)-\mathrm{P}\left(4^{\prime}\right)$ & $120.0(14)$ \\
\hline $\mathrm{C}(21)-\mathrm{C}(20)-\mathrm{C}(19)$ & $120.4(16)$ & $\mathrm{C}\left(33^{\prime}\right)-\mathrm{C}\left(32^{\prime}\right)-\mathrm{C}\left(31^{\prime}\right)$ & $118.0(16)$ \\
\hline $\mathrm{C}\left(22^{\prime}\right)-\mathrm{C}\left(21^{\prime}\right)-\mathrm{C}\left(20^{\prime}\right)$ & $118.9(17)$ & $\mathrm{C}(33)-\mathrm{C}(32)-\mathrm{C}(31)$ & $118.4(13)$ \\
\hline $\mathrm{C}(22)-\mathrm{C}(21)-\mathrm{C}(20)$ & $122.3(18)$ & $\mathrm{C}(33)-\mathrm{C}(32)-\mathrm{P}(4)$ & $123.1(10)$ \\
\hline $\mathrm{C}(21)-\mathrm{C}(22)-\mathrm{C}(23)$ & $117.2(16)$ & $\mathrm{C}(31)-\mathrm{C}(32)-\mathrm{P}(4)$ & $118.4(10)$ \\
\hline $\mathrm{C}\left(21^{\prime}\right)-\mathrm{C}\left(22^{\prime}\right)-\mathrm{C}\left(23^{\prime}\right)$ & $121.7(19)$ & $\mathrm{C}(32)-\mathrm{C}(33)-\mathrm{C}(34)$ & $123.3(14)$ \\
\hline
\end{tabular}




\begin{tabular}{|c|c|c|c|}
\hline $\mathrm{C}\left(32^{\prime}\right)-\mathrm{C}\left(33^{\prime}\right)-\mathrm{C}\left(34^{\prime}\right)$ & $122.7(16)$ & $\mathrm{C}\left(42^{\prime}\right)-\mathrm{C}\left(41^{\prime}\right)-\mathrm{P}\left(3^{\prime}\right)$ & $110.1(14)$ \\
\hline $\mathrm{C}\left(33^{\prime}\right)-\mathrm{C}\left(34^{\prime}\right)-\mathrm{C}\left(35^{\prime}\right)$ & $117.7(18)$ & $\mathrm{C}\left(48^{\prime}\right)-\mathrm{C}\left(43^{\prime}\right)-\mathrm{C}\left(44^{\prime}\right)$ & $116.1(18)$ \\
\hline $\mathrm{C}(33)-\mathrm{C}(34)-\mathrm{C}(35)$ & $117.7(15)$ & $\mathrm{C}\left(48^{\prime}\right)-\mathrm{C}\left(43^{\prime}\right)-\operatorname{Pt}\left(2^{\prime}\right)$ & $129.0(15)$ \\
\hline $\mathrm{C}\left(36^{\prime}\right)-\mathrm{C}\left(35^{\prime}\right)-\mathrm{C}\left(34^{\prime}\right)$ & 119.3(19) & $\mathrm{C}\left(44^{\prime}\right)-\mathrm{C}\left(43^{\prime}\right)-\operatorname{Pt}\left(2^{\prime}\right)$ & $114.9(14)$ \\
\hline $\mathrm{C}(36)-\mathrm{C}(35)-\mathrm{C}(34)$ & $121.4(15)$ & $\mathrm{C}(48)-\mathrm{C}(43)-\mathrm{C}(47)$ & 116.1(19) \\
\hline $\mathrm{C}\left(31^{\prime}\right)-\mathrm{C}\left(36^{\prime}\right)-\mathrm{C}\left(35^{\prime}\right)$ & 121.1(16) & $\mathrm{C}(48)-\mathrm{C}(43)-\mathrm{Pt}(2)$ & $117.6(15)$ \\
\hline $\mathrm{C}\left(31^{\prime}\right)-\mathrm{C}\left(36^{\prime}\right)-\mathrm{P}\left(3^{\prime}\right)$ & $117.5(12)$ & $\mathrm{C}(47)-\mathrm{C}(43)-\mathrm{Pt}(2)$ & $126.2(15)$ \\
\hline $\mathrm{C}\left(35^{\prime}\right)-\mathrm{C}\left(36^{\prime}\right)-\mathrm{P}\left(3^{\prime}\right)$ & $121.2(13)$ & $\mathrm{C}(48)-\mathrm{C}(44)-\mathrm{C}(45)$ & 121(3) \\
\hline $\mathrm{C}(35)-\mathrm{C}(36)-\mathrm{C}(31)$ & $121.1(15)$ & $\mathrm{C}\left(45^{\prime}\right)-\mathrm{C}\left(44^{\prime}\right)-\mathrm{C}\left(43^{\prime}\right)$ & $118(2)$ \\
\hline $\mathrm{C}\left(37^{\prime}\right)-\mathrm{C}\left(38^{\prime}\right)-\mathrm{C}\left(39^{\prime}\right)$ & $119.3(17)$ & $\mathrm{C}(46)-\mathrm{C}(45)-\mathrm{C}(44)$ & $115.9(18)$ \\
\hline $\mathrm{C}\left(37^{\prime}\right)-\mathrm{C}\left(38^{\prime}\right)-\mathrm{P}\left(3^{\prime}\right)$ & 111.6(13) & $\mathrm{C}\left(44^{\prime}\right)-\mathrm{C}\left(45^{\prime}\right)-\mathrm{C}\left(46^{\prime}\right)$ & $120(2)$ \\
\hline $\mathrm{C}\left(39^{\prime}\right)-\mathrm{C}\left(38^{\prime}\right)-\mathrm{P}\left(3^{\prime}\right)$ & 101.3(13) & $\mathrm{C}(47)-\mathrm{C}(46)-\mathrm{C}(45)$ & $126(2)$ \\
\hline $\mathrm{C}(39)-\mathrm{C}(38)-\mathrm{C}(37)$ & $122(3)$ & $\mathrm{C}\left(47^{\prime}\right)-\mathrm{C}\left(46^{\prime}\right)-\mathrm{C}\left(45^{\prime}\right)$ & $118(2)$ \\
\hline C(39)-C(38)-P(3) & $102(2)$ & $\mathrm{C}(46)-\mathrm{C}(47)-\mathrm{C}(43)$ & $120(2)$ \\
\hline C(37)-C(38)-P(3) & 109.7(19) & $\mathrm{C}\left(46^{\prime}\right)-\mathrm{C}\left(47^{\prime}\right)-\mathrm{C}\left(48^{\prime}\right)$ & $117(3)$ \\
\hline $\mathrm{C}\left(40^{\prime}\right)-\mathrm{C}\left(39^{\prime}\right)-\mathrm{C}\left(38^{\prime}\right)$ & $109.8(16)$ & $\mathrm{C}(44)-\mathrm{C}(48)-\mathrm{C}(43)$ & $120(3)$ \\
\hline $\mathrm{C}(40)-\mathrm{C}(39)-\mathrm{C}(38)$ & $105(3)$ & $\mathrm{C}\left(43^{\prime}\right)-\mathrm{C}\left(48^{\prime}\right)-\mathrm{C}\left(47^{\prime}\right)$ & $127(2)$ \\
\hline $\mathrm{C}\left(39^{\prime}\right)-\mathrm{C}\left(40^{\prime}\right)-\mathrm{C}\left(41^{\prime}\right)$ & $110.5(17)$ & $\mathrm{O}\left(3^{\prime}\right)-\mathrm{C}\left(49^{\prime}\right)-\mathrm{O}\left(1^{\prime}\right)$ & $125.6(18)$ \\
\hline$C(39)-C(40)-C(41)$ & $109(3)$ & $\mathrm{O}\left(3^{\prime}\right)-\mathrm{C}\left(49^{\prime}\right)-\mathrm{O}\left(2^{\prime}\right)$ & $125.5(17)$ \\
\hline $\mathrm{C}(42)-\mathrm{C}(41)-\mathrm{C}(40)$ & $115(3)$ & $\mathrm{O}\left(1^{\prime}\right)-\mathrm{C}\left(49^{\prime}\right)-\mathrm{O}\left(2^{\prime}\right)$ & $108.9(17)$ \\
\hline C(42)-C(41)-P(3) & $111(2)$ & $\mathrm{O}(2)-\mathrm{C}(49)-\mathrm{O}(3)$ & $129.6(14)$ \\
\hline $\mathrm{C}(40)-\mathrm{C}(41)-\mathrm{P}(3)$ & $96(2)$ & $\mathrm{O}(2)-\mathrm{C}(49)-\mathrm{O}(1)$ & $117.9(14)$ \\
\hline$C\left(40^{\prime}\right)-C\left(41^{\prime}\right)-C\left(42^{\prime}\right)$ & $115.7(18)$ & $\mathrm{O}(3)-\mathrm{C}(49)-\mathrm{O}(1)$ & $112.2(13)$ \\
\hline $\mathrm{C}\left(40^{\prime}\right)-\mathrm{C}\left(41^{\prime}\right)-\mathrm{P}\left(3^{\prime}\right)$ & $104.2(14)$ & & \\
\hline
\end{tabular}

Symmetry transformations used to generate equivalent atoms: 
Table 4. Anisotropic displacement parameters $\left(\AA^{2} \mathrm{x} 10^{3}\right)$ for $[\mathrm{Pt}(\mathrm{Me}-\mathrm{Duphos})(\mathrm{Ph})]_{2}\left(\mu-\mathrm{CO}_{3}\right)$, glu220. The anisotropic displacement factor exponent takes the form: $-2 \pi^{2}\left[h^{2} a^{* 2} U^{11}+\ldots+2 h k a^{*} b^{*} U^{12}\right]$

\begin{tabular}{|c|c|c|c|c|c|c|}
\hline & $\mathrm{U}^{11}$ & $\mathrm{U}^{22}$ & $\mathrm{U}^{33}$ & $\mathrm{U}^{23}$ & $\mathrm{U}^{13}$ & $\mathrm{U}^{12}$ \\
\hline $\operatorname{Pt}(1)$ & $34(1)$ & $30(1)$ & $24(1)$ & $-1(1)$ & $0(1)$ & $-6(1)$ \\
\hline $\operatorname{Pt}(2)$ & $33(1)$ & $32(1)$ & $29(1)$ & $-3(1)$ & $-1(1)$ & $-5(1)$ \\
\hline $\operatorname{Pt}\left(1^{\prime}\right)$ & $37(1)$ & $40(1)$ & $30(1)$ & $-4(1)$ & $2(1)$ & $-9(1)$ \\
\hline $\operatorname{Pt}\left(2^{\prime}\right)$ & $39(1)$ & $38(1)$ & $30(1)$ & $-2(1)$ & $4(1)$ & $-8(1)$ \\
\hline $\mathrm{P}(1)$ & $38(2)$ & $33(2)$ & $30(2)$ & $-3(2)$ & $1(2)$ & $-12(2)$ \\
\hline $\mathrm{P}\left(1^{\prime}\right)$ & $29(2)$ & $48(2)$ & $34(2)$ & $1(2)$ & $2(2)$ & $-2(2)$ \\
\hline $\mathrm{P}(2)$ & $34(2)$ & $37(2)$ & $27(2)$ & $-3(2)$ & $-2(2)$ & $-6(2)$ \\
\hline $\mathrm{P}\left(2^{\prime}\right)$ & $43(2)$ & $38(2)$ & $38(2)$ & $-4(2)$ & $8(2)$ & $-10(2)$ \\
\hline $\mathrm{P}(3)$ & $38(2)$ & $40(2)$ & $34(2)$ & $-2(2)$ & $0(2)$ & $-14(2)$ \\
\hline $\mathrm{P}\left(3^{\prime}\right)$ & $46(2)$ & $39(2)$ & $35(2)$ & $-3(2)$ & $3(2)$ & $-7(2)$ \\
\hline $\mathrm{P}(4)$ & $29(2)$ & $32(2)$ & $32(2)$ & $1(2)$ & $-8(2)$ & $-3(2)$ \\
\hline $\mathrm{P}\left(4^{\prime}\right)$ & $41(2)$ & $47(2)$ & $28(2)$ & $-6(2)$ & $3(2)$ & $-11(2)$ \\
\hline $\mathrm{O}(1)$ & $42(6)$ & $45(6)$ & $48(7)$ & $-7(5)$ & $-2(5)$ & $-36(5)$ \\
\hline $\mathrm{O}\left(1^{\prime}\right)$ & $89(10)$ & $62(8)$ & $55(8)$ & 21(7) & $-17(8)$ & $-49(8)$ \\
\hline $\mathrm{O}\left(2^{\prime}\right)$ & $52(7)$ & $39(6)$ & 73(9) & $1(6)$ & $30(7)$ & $-14(5)$ \\
\hline $\mathrm{O}(2)$ & $41(7)$ & $61(8)$ & $63(8)$ & $-4(7)$ & $-4(6)$ & $-7(6)$ \\
\hline $\mathrm{O}\left(3^{\prime}\right)$ & $52(8)$ & $67(9)$ & $95(12)$ & $2(9)$ & $19(8)$ & $3(7)$ \\
\hline $\mathrm{O}(3)$ & $52(7)$ & $19(5)$ & $44(6)$ & $-11(4)$ & $0(5)$ & $-24(5)$ \\
\hline$C(1)$ & $78(16)$ & $66(14)$ & $87(17)$ & $-1(13)$ & $28(13)$ & $-5(12)$ \\
\hline$C\left(1^{\prime}\right)$ & $87(17)$ & $45(11)$ & $92(18)$ & $-5(11)$ & $7(14)$ & $2(11)$ \\
\hline$C(2)$ & $88(14)$ & $44(10)$ & $41(10)$ & $-16(8)$ & $32(10)$ & $-19(10)$ \\
\hline$C\left(2^{\prime}\right)$ & $76(13)$ & $51(10)$ & $44(10)$ & $-10(8)$ & $11(10)$ & $-9(10)$ \\
\hline$C\left(3^{\prime}\right)$ & 108(19) & $46(11)$ & $56(13)$ & $-26(10)$ & $5(12)$ & $-16(11)$ \\
\hline$C(3)$ & $160(30)$ & $69(14)$ & $38(11)$ & $-9(10)$ & $33(15)$ & $-16(17)$ \\
\hline$C\left(4^{\prime}\right)$ & $98(17)$ & $97(18)$ & $31(10)$ & $-5(11)$ & $-1(10)$ & $-18(16)$ \\
\hline$C(4)$ & 108(19) & $75(15)$ & $39(11)$ & $11(10)$ & $-15(12)$ & $-50(14)$ \\
\hline$C(5)$ & $46(9)$ & $48(10)$ & 40(9) & $3(8)$ & $-10(8)$ & $-16(8)$ \\
\hline$C\left(5^{\prime}\right)$ & $36(9)$ & $68(12)$ & $36(9)$ & $12(9)$ & $-10(7)$ & $-7(8)$ \\
\hline$C\left(6^{\prime}\right)$ & $33(10)$ & $74(14)$ & $88(16)$ & $19(12)$ & $10(10)$ & $16(9)$ \\
\hline
\end{tabular}




\begin{tabular}{|c|c|c|c|c|c|c|}
\hline$C(6)$ & $38(9)$ & $64(12)$ & $47(10)$ & $13(9)$ & $-2(8)$ & $5(8)$ \\
\hline$C\left(7^{\prime}\right)$ & $56(10)$ & $36(8)$ & $17(7)$ & $11(6)$ & $9(7)$ & $19(7)$ \\
\hline$C(7)$ & $36(8)$ & $37(8)$ & $25(7)$ & $1(6)$ & $12(6)$ & $-15(6)$ \\
\hline$C\left(8^{\prime}\right)$ & $59(11)$ & $58(10)$ & $17(7)$ & $-4(7)$ & $10(7)$ & $12(9)$ \\
\hline$C(8)$ & $69(13)$ & $63(12)$ & $39(10)$ & 18(9) & $1(9)$ & $-12(10)$ \\
\hline$C\left(9^{\prime}\right)$ & $38(9)$ & $51(10)$ & $60(12)$ & $-3(9)$ & $10(9)$ & $17(8)$ \\
\hline$C(9)$ & $57(11)$ & $51(10)$ & $45(10)$ & $21(8)$ & $16(9)$ & 2(9) \\
\hline $\mathrm{C}\left(10^{\prime}\right)$ & $38(9)$ & $31(8)$ & $67(12)$ & $0(8)$ & 11(8) & $-5(7)$ \\
\hline$C(10)$ & $65(11)$ & $35(8)$ & $45(10)$ & 11(7) & $13(9)$ & $-21(8)$ \\
\hline$C\left(11^{\prime}\right)$ & $38(9)$ & $47(9)$ & $52(10)$ & $10(8)$ & $0(8)$ & $-8(7)$ \\
\hline $\mathrm{C}(11)$ & $33(8)$ & $39(8)$ & $49(10)$ & $2(7)$ & $2(7)$ & $-9(7)$ \\
\hline$C\left(12^{\prime}\right)$ & $45(9)$ & $36(7)$ & $31(8)$ & $-6(6)$ & $33(7)$ & $8(6)$ \\
\hline $\mathrm{C}(12)$ & 41(9) & $40(9)$ & $62(12)$ & $15(8)$ & $-4(8)$ & $-12(8)$ \\
\hline$C(13)$ & $62(13)$ & $49(12)$ & $150(30)$ & $-38(14)$ & $47(15)$ & $3(10)$ \\
\hline$C\left(13^{\prime}\right)$ & $64(14)$ & $57(13)$ & $180(30)$ & $7(16)$ & $75(18)$ & $26(11)$ \\
\hline$C(14)$ & 119(19) & $72(14)$ & $39(10)$ & $-30(9)$ & $36(12)$ & $-72(15)$ \\
\hline$C\left(14^{\prime}\right)$ & $52(11)$ & $26(7)$ & $85(14)$ & $-20(8)$ & $22(10)$ & $-21(7)$ \\
\hline$C(15)$ & $57(11)$ & $60(11)$ & $46(11)$ & $-10(9)$ & $-4(9)$ & $-23(9)$ \\
\hline$C\left(15^{\prime}\right)$ & $110(20)$ & $68(14)$ & $54(13)$ & $-34(11)$ & $33(13)$ & $-44(15)$ \\
\hline$C(16)$ & $60(13)$ & $78(15)$ & $74(15)$ & $4(12)$ & $-37(12)$ & $-23(11)$ \\
\hline$C\left(16^{\prime}\right)$ & $130(30)$ & $74(15)$ & $46(13)$ & $-13(11)$ & $4(14)$ & $-24(16)$ \\
\hline $\mathrm{C}(17)$ & $26(8)$ & $56(11)$ & $72(13)$ & $9(9)$ & $-12(8)$ & $-17(7)$ \\
\hline$C\left(17^{\prime}\right)$ & $42(10)$ & $72(14)$ & $54(12)$ & $18(10)$ & $-21(9)$ & $-20(9)$ \\
\hline$C(18)$ & $36(9)$ & 73(13) & $85(16)$ & $13(12)$ & $-2(10)$ & $20(9)$ \\
\hline$C\left(18^{\prime}\right)$ & $230(50)$ & $110(20)$ & $110(30)$ & $40(20)$ & $-110(30)$ & $-60(30)$ \\
\hline$C(19)$ & $32(7)$ & $29(7)$ & $23(7)$ & $1(5)$ & $-1(6)$ & $1(6)$ \\
\hline $\mathrm{C}\left(19^{\prime}\right)$ & $53(9)$ & $54(9)$ & $14(6)$ & $3(6)$ & $-3(6)$ & $-21(8)$ \\
\hline$C\left(20^{\prime}\right)$ & $55(11)$ & $38(9)$ & $46(10)$ & $-5(7)$ & $14(8)$ & $1(8)$ \\
\hline $\mathrm{C}(20)$ & 41(9) & $51(10)$ & $39(9)$ & $12(8)$ & $14(7)$ & $15(8)$ \\
\hline$C\left(21^{\prime}\right)$ & $46(9)$ & $60(11)$ & $35(9)$ & $-7(8)$ & $18(7)$ & $5(8)$ \\
\hline$C(21)$ & $62(11)$ & $47(9)$ & $30(9)$ & $-12(7)$ & $14(8)$ & $4(8)$ \\
\hline$C(22)$ & $93(15)$ & $38(9)$ & $29(8)$ & $-2(7)$ & $0(9)$ & $-8(10)$ \\
\hline$C\left(22^{\prime}\right)$ & $130(20)$ & $43(11)$ & $36(10)$ & $-3(8)$ & $10(12)$ & $18(12)$ \\
\hline
\end{tabular}




\begin{tabular}{|c|c|c|c|c|c|c|}
\hline$C(23)$ & $68(12)$ & $48(10)$ & $24(8)$ & $4(7)$ & $-10(8)$ & $-19(9)$ \\
\hline$C\left(23^{\prime}\right)$ & $115(19)$ & $47(10)$ & $38(10)$ & $-2(8)$ & $28(11)$ & $28(12)$ \\
\hline$C(24)$ & $63(10)$ & $31(7)$ & $34(8)$ & $-4(7)$ & $-2(7)$ & $-15(8)$ \\
\hline$C\left(24^{\prime}\right)$ & $63(11)$ & $47(10)$ & $33(9)$ & $-1(7)$ & $20(8)$ & $3(8)$ \\
\hline$C(25)$ & $41(10)$ & $60(11)$ & $73(14)$ & $6(10)$ & $-28(10)$ & $-6(9)$ \\
\hline$C\left(25^{\prime}\right)$ & $79(16)$ & $89(16)$ & $62(14)$ & $22(13)$ & $-28(12)$ & $-1(13)$ \\
\hline$C(26)$ & $36(9)$ & $50(9)$ & $54(11)$ & $-5(8)$ & $-13(8)$ & $11(8)$ \\
\hline$C\left(26^{\prime}\right)$ & $53(11)$ & $73(13)$ & $49(11)$ & $2(9)$ & $12(9)$ & $-2(10)$ \\
\hline$C\left(27^{\prime}\right)$ & $100(20)$ & $120(20)$ & $54(14)$ & $3(14)$ & $-32(14)$ & $-52(18)$ \\
\hline $\mathrm{C}(27)$ & 41(9) & $61(11)$ & $46(10)$ & $-3(8)$ & $-15(8)$ & $-5(8)$ \\
\hline$C\left(28^{\prime}\right)$ & $70(13)$ & $47(10)$ & $57(12)$ & $-22(9)$ & $0(10)$ & $-1(9)$ \\
\hline $\mathrm{C}(28)$ & $58(11)$ & $75(12)$ & $27(8)$ & $4(8)$ & $-15(8)$ & $-6(10)$ \\
\hline $\mathrm{C}(29)$ & $59(11)$ & $31(8)$ & $45(10)$ & $-9(7)$ & $-8(8)$ & $-3(7)$ \\
\hline$C\left(29^{\prime}\right)$ & $56(11)$ & $55(10)$ & $50(11)$ & $-24(9)$ & $10(9)$ & $-23(9)$ \\
\hline$C(30)$ & $34(9)$ & $60(11)$ & $67(12)$ & $-22(10)$ & $9(9)$ & $3(8)$ \\
\hline$C\left(30^{\prime}\right)$ & $72(15)$ & $44(11)$ & $140(30)$ & $14(13)$ & $-8(15)$ & $17(10)$ \\
\hline $\mathrm{C}(31)$ & $26(7)$ & $42(8)$ & $25(7)$ & $11(6)$ & $-5(6)$ & $2(6)$ \\
\hline $\mathrm{C}\left(31^{\prime}\right)$ & $23(8)$ & $54(9)$ & $61(11)$ & $-5(8)$ & $14(7)$ & $-21(7)$ \\
\hline$C\left(32^{\prime}\right)$ & $44(10)$ & $62(10)$ & $38(9)$ & $-5(8)$ & $5(8)$ & $-20(9)$ \\
\hline$C(32)$ & $31(7)$ & $25(7)$ & $28(7)$ & $-8(5)$ & $-2(6)$ & $-5(6)$ \\
\hline$C(33)$ & $30(7)$ & $39(8)$ & $19(7)$ & $-3(6)$ & $-11(5)$ & $-1(6)$ \\
\hline$C\left(33^{\prime}\right)$ & $57(11)$ & $61(12)$ & $53(12)$ & $-2(9)$ & $15(9)$ & $-29(10)$ \\
\hline$C\left(34^{\prime}\right)$ & $46(11)$ & $78(14)$ & $68(14)$ & $15(11)$ & $13(10)$ & $-35(10)$ \\
\hline$C(34)$ & $39(9)$ & $51(10)$ & $40(9)$ & $-4(7)$ & 1(7) & $-2(7)$ \\
\hline$C\left(35^{\prime}\right)$ & 49(11) & $63(11)$ & $55(11)$ & $-9(9)$ & $30(9)$ & $-3(9)$ \\
\hline$C(35)$ & $39(8)$ & $58(10)$ & $22(7)$ & $17(7)$ & $12(6)$ & $0(8)$ \\
\hline$C\left(36^{\prime}\right)$ & $60(11)$ & $26(7)$ & $40(9)$ & $-13(6)$ & $9(8)$ & $-9(7)$ \\
\hline$C(36)$ & 49(10) & $36(8)$ & $42(9)$ & $4(7)$ & $-12(8)$ & $-9(8)$ \\
\hline$C\left(37^{\prime}\right)$ & $36(10)$ & $78(14)$ & $101(18)$ & $36(13)$ & $-28(11)$ & $-8(10)$ \\
\hline$C(37)$ & $26(11)$ & $90(20)$ & $260(50)$ & $70(20)$ & $14(17)$ & $17(11)$ \\
\hline$C\left(38^{\prime}\right)$ & 44(9) & $57(11)$ & $35(9)$ & $-1(8)$ & $-1(7)$ & $0(8)$ \\
\hline $\mathrm{C}(38)$ & 43(10) & $180(30)$ & $38(11)$ & $54(15)$ & $-22(8)$ & $-7(15)$ \\
\hline$C\left(39^{\prime}\right)$ & $78(16)$ & $130(20)$ & $24(9)$ & $-9(11)$ & $-11(9)$ & $-58(16)$ \\
\hline
\end{tabular}




\begin{tabular}{lcccccc}
$\mathrm{C}(39)$ & $210(50)$ & $260(60)$ & $70(20)$ & $50(30)$ & $-70(30)$ & $-200(50)$ \\
$\mathrm{C}\left(40^{\prime}\right)$ & $99(18)$ & $50(11)$ & $45(12)$ & $-13(9)$ & $10(11)$ & $-13(12)$ \\
$\mathrm{C}(40)$ & $390(90)$ & $190(40)$ & $38(17)$ & $-50(20)$ & $50(30)$ & $-240(60)$ \\
$\mathrm{C}(41)$ & $170(30)$ & $43(11)$ & $41(11)$ & $-17(9)$ & $50(14)$ & $-38(13)$ \\
$\mathrm{C}\left(41^{\prime}\right)$ & $50(10)$ & $58(11)$ & $35(9)$ & $-10(8)$ & $4(8)$ & $1(9)$ \\
$\mathrm{C}\left(42^{\prime}\right)$ & $110(20)$ & $70(14)$ & $63(14)$ & $7(11)$ & $14(14)$ & $30(14)$ \\
$\mathrm{C}(42)$ & $500(100)$ & $44(15)$ & $100(30)$ & $32(16)$ & $120(40)$ & $80(30)$ \\
$\mathrm{C}\left(43^{\prime}\right)$ & $30(7)$ & $60(10)$ & $31(8)$ & $1(8)$ & $0(6)$ & $-3(7)$ \\
$\mathrm{C}(43)$ & $45(9)$ & $33(8)$ & $53(10)$ & $-18(7)$ & $3(8)$ & $-17(7)$ \\
$\mathrm{C}(44)$ & $140(30)$ & $81(17)$ & $70(17)$ & $-41(14)$ & $56(18)$ & $-72(18)$ \\
$\mathrm{C}\left(44^{\prime}\right)$ & $68(13)$ & $67(13)$ & $46(11)$ & $10(9)$ & $-7(10)$ & $-10(10)$ \\
$\mathrm{C}(45)$ & $160(30)$ & $86(16)$ & $14(9)$ & $3(9)$ & $-7(13)$ & $-64(18)$ \\
$\mathrm{C}\left(45^{\prime}\right)$ & $59(13)$ & $120(20)$ & $58(14)$ & $44(14)$ & $-12(11)$ & $-5(13)$ \\
$\mathrm{C}(46)$ & $120(20)$ & $67(14)$ & $34(11)$ & $18(10)$ & $-17(12)$ & $-8(14)$ \\
$\mathrm{C}\left(46^{\prime}\right)$ & $190(30)$ & $91(19)$ & $26(10)$ & $-9(11)$ & $40(15)$ & $-40(20)$ \\
$\mathrm{C}(47)$ & $68(14)$ & $73(14)$ & $57(13)$ & $-10(11)$ & $-5(11)$ & $-2(11)$ \\
$\mathrm{C}\left(47^{\prime}\right)$ & $63(13)$ & $115(19)$ & $41(11)$ & $7(12)$ & $-13(10)$ & $-10(14)$ \\
$\mathrm{C}(48)$ & $101(17)$ & $39(9)$ & $49(11)$ & $-12(8)$ & $31(11)$ & $-28(10)$ \\
$\mathrm{C}\left(48^{\prime}\right)$ & $29(8)$ & $102(15)$ & $34(9)$ & $-27(10)$ & $6(7)$ & $-15(9)$ \\
$\mathrm{C}\left(49^{\prime}\right)$ & $22(7)$ & $61(10)$ & $40(9)$ & $-10(8)$ & $18(7)$ & $-17(8)$ \\
$\mathrm{C}(49)$ & $39(8)$ & $29(7)$ & $30(8)$ & $1(6)$ & $-20(7)$ & $6(6)$ \\
\hline & & & & & \\
\hline
\end{tabular}


Table 5. Hydrogen coordinates (x $\left.10^{4}\right)$ and isotropic displacement parameters $\left(\AA^{2} \times 10^{3}\right)$ for $[\mathrm{Pt}(\mathrm{Me}-\mathrm{Duphos})(\mathrm{Ph})]_{2}\left(\mu-\mathrm{CO}_{3}\right)$, glu220.

\begin{tabular}{|c|c|c|c|c|}
\hline & $\mathrm{x}$ & $\mathrm{y}$ & $\mathrm{z}$ & $\mathrm{U}(\mathrm{eq})$ \\
\hline $\mathrm{H}(1 \mathrm{~A})$ & 5454 & 5920 & 6672 & 116 \\
\hline $\mathrm{H}(1 \mathrm{~B})$ & 5563 & 6334 & 7023 & 116 \\
\hline $\mathrm{H}(1 \mathrm{C})$ & 5417 & 6874 & 6704 & 116 \\
\hline $\mathrm{H}\left(1^{\prime} \mathrm{A}\right)$ & 1002 & 10776 & 10590 & 112 \\
\hline $\mathrm{H}\left(1^{\prime} \mathrm{B}\right)$ & 613 & 10208 & 10314 & 112 \\
\hline $\mathrm{H}\left(1^{\prime} \mathrm{C}\right)$ & 1582 & 10422 & 10307 & 112 \\
\hline $\mathrm{H}(2 \mathrm{~A})$ & 4191 & 5799 & 6981 & 69 \\
\hline $\mathrm{H}\left(2^{\prime} \mathrm{A}\right)$ & 824 & 9466 & 10820 & 68 \\
\hline $\mathrm{H}\left(3^{\prime} \mathrm{A}\right)$ & 2579 & 9881 & 10718 & 84 \\
\hline $\mathrm{H}\left(3^{\prime} \mathrm{B}\right)$ & 2054 & 10060 & 11046 & 84 \\
\hline $\mathrm{H}(3 \mathrm{~A})$ & 3891 & 6742 & 6408 & 105 \\
\hline $\mathrm{H}(3 \mathrm{~B})$ & 3671 & 5818 & 6463 & 105 \\
\hline $\mathrm{H}\left(4^{\prime} \mathrm{A}\right)$ & 1917 & 8661 & 11152 & 91 \\
\hline $\mathrm{H}\left(4^{\prime} \mathrm{B}\right)$ & 2889 & 8846 & 11106 & 91 \\
\hline $\mathrm{H}(4 \mathrm{~A})$ & 2569 & 6136 & 6802 & 89 \\
\hline $\mathrm{H}(4 \mathrm{~B})$ & 2477 & 6697 & 6483 & 89 \\
\hline $\mathrm{H}(5 \mathrm{~A})$ & 3083 & 7763 & 6737 & 54 \\
\hline $\mathrm{H}\left(5^{\prime} \mathrm{A}\right)$ & 2914 & 8341 & 10584 & 56 \\
\hline $\mathrm{H}\left(6^{\prime} \mathrm{A}\right)$ & 2825 & 7252 & 10935 & 98 \\
\hline $\mathrm{H}\left(6^{\prime} \mathrm{B}\right)$ & 2358 & 7044 & 10599 & 98 \\
\hline $\mathrm{H}\left(6^{\prime} \mathrm{C}\right)$ & 1831 & 7272 & 10919 & 98 \\
\hline $\mathrm{H}(6 \mathrm{~A})$ & 1704 & 7648 & 6942 & 74 \\
\hline $\mathrm{H}(6 \mathrm{~B})$ & 2298 & 8049 & 7210 & 74 \\
\hline $\mathrm{H}(6 \mathrm{C})$ & 2035 & 7130 & 7244 & 74 \\
\hline $\mathrm{H}\left(8^{\prime} \mathrm{A}\right)$ & 345 & 8138 & 10983 & 54 \\
\hline $\mathrm{H}(8 \mathrm{~A})$ & 4509 & 8241 & 6623 & 69 \\
\hline $\mathrm{H}\left(9^{\prime} \mathrm{A}\right)$ & -775 & 7277 & 10998 & 60 \\
\hline $\mathrm{H}(9 \mathrm{~A})$ & 5291 & 9413 & 6636 & 61 \\
\hline $\mathrm{H}(10 \mathrm{~B})$ & -1243 & 6652 & 10536 & 54 \\
\hline
\end{tabular}




\begin{tabular}{|c|c|c|c|c|}
\hline $\mathrm{H}(10 \mathrm{~A})$ & 5861 & 9880 & 7112 & 58 \\
\hline $\mathrm{H}(11 \mathrm{~B})$ & -610 & 6900 & 10030 & 55 \\
\hline $\mathrm{H}(11 \mathrm{~A})$ & 5645 & 9175 & 7619 & 48 \\
\hline $\mathrm{H}(13 \mathrm{~A})$ & 3951 & 9488 & 8264 & 128 \\
\hline $\mathrm{H}(13 \mathrm{~B})$ & 3484 & 9050 & 7968 & 128 \\
\hline $\mathrm{H}(13 \mathrm{C})$ & 4356 & 9487 & 7906 & 128 \\
\hline $\mathrm{H}(13 \mathrm{D})$ & 2043 & 5999 & 9581 & 150 \\
\hline $\mathrm{H}(13 \mathrm{E})$ & 2147 & 6557 & 9897 & 150 \\
\hline $\mathrm{H}(13 \mathrm{~F})$ & 2299 & 6919 & 9538 & 150 \\
\hline $\mathrm{H}(14 \mathrm{~A})$ & 4080 & 8146 & 8290 & 92 \\
\hline $\mathrm{H}(15 \mathrm{~A})$ & 5158 & 8657 & 8552 & 65 \\
\hline $\mathrm{H}(15 \mathrm{~B})$ & 5605 & 8952 & 8222 & 65 \\
\hline $\mathrm{H}(15 \mathrm{C})$ & 983 & 7076 & 9141 & 94 \\
\hline $\mathrm{H}(15 \mathrm{D})$ & 580 & 6219 & 9217 & 94 \\
\hline $\mathrm{H}(16 \mathrm{~A})$ & 5478 & 7313 & 8439 & 85 \\
\hline $\mathrm{H}(16 \mathrm{~B})$ & 6328 & 7800 & 8379 & 85 \\
\hline $\mathrm{H}(16 \mathrm{C})$ & -538 & 6798 & 9457 & 101 \\
\hline $\mathrm{H}(16 \mathrm{D})$ & -411 & 7203 & 9104 & 101 \\
\hline $\mathrm{H}(17 \mathrm{~A})$ & 6129 & 7845 & 7819 & 61 \\
\hline $\mathrm{H}(18 \mathrm{~A})$ & 6568 & 6521 & 7935 & 97 \\
\hline $\mathrm{H}(18 \mathrm{~B})$ & 5992 & 6537 & 7616 & 97 \\
\hline $\mathrm{H}(18 \mathrm{C})$ & 5620 & 6237 & 7958 & 97 \\
\hline $\mathrm{H}(18 \mathrm{D})$ & -1100 & 8434 & 9398 & 223 \\
\hline $\mathrm{H}(18 \mathrm{E})$ & -589 & 8924 & 9668 & 223 \\
\hline $\mathrm{H}(18 \mathrm{~F})$ & -1009 & 8086 & 9762 & 223 \\
\hline $\mathrm{H}(20 \mathrm{~B})$ & 2362 & 7898 & 9189 & 56 \\
\hline $\mathrm{H}(20 \mathrm{~A})$ & 2387 & 6994 & 8205 & 53 \\
\hline $\mathrm{H}(21 \mathrm{~B})$ & 2569 & 8295 & 8642 & 56 \\
\hline $\mathrm{H}(21 \mathrm{~A})$ & 2111 & 6712 & 8751 & 55 \\
\hline $\mathrm{H}(22 \mathrm{~A})$ & 3008 & 5932 & 9062 & 64 \\
\hline $\mathrm{H}(22 \mathrm{~B})$ & 2247 & 9588 & 8489 & 84 \\
\hline $\mathrm{H}(23 \mathrm{~A})$ & 4338 & 5625 & 8835 & 56 \\
\hline $\mathrm{H}(23 \mathrm{~B})$ & 1374 & 10382 & 8831 & 80 \\
\hline
\end{tabular}




\begin{tabular}{|c|c|c|c|c|}
\hline $\mathrm{H}(24 \mathrm{~A})$ & 4659 & 6021 & 8299 & 51 \\
\hline $\mathrm{H}(24 \mathrm{~B})$ & 1394 & 10082 & 9392 & 57 \\
\hline $\mathrm{H}(25 \mathrm{~A})$ & -1699 & 3389 & 7019 & 87 \\
\hline $\mathrm{H}(25 \mathrm{~B})$ & -1133 & 3793 & 7296 & 87 \\
\hline $\mathrm{H}(25 \mathrm{C})$ & -1250 & 2842 & 7288 & 87 \\
\hline $\mathrm{H}(25 \mathrm{D})$ & 6878 & 10624 & 10868 & 115 \\
\hline $\mathrm{H}(25 \mathrm{E})$ & 6593 & 10418 & 10502 & 115 \\
\hline $\mathrm{H}(25 \mathrm{~F})$ & 5912 & 10514 & 10785 & 115 \\
\hline $\mathrm{H}(26 \mathrm{~A})$ & -418 & 3757 & 6807 & 56 \\
\hline $\mathrm{H}(26 \mathrm{~B})$ & 6928 & 11773 & 10536 & 70 \\
\hline $\mathrm{H}(27 \mathrm{C})$ & 6705 & 12164 & 11061 & 110 \\
\hline $\mathrm{H}(27 \mathrm{D})$ & 5748 & 11880 & 11055 & 110 \\
\hline $\mathrm{H}(27 \mathrm{~A})$ & -844 & 2684 & 6505 & 59 \\
\hline $\mathrm{H}(27 \mathrm{~B})$ & -682 & 2063 & 6799 & 59 \\
\hline $\mathrm{H}(28 \mathrm{C})$ & 5665 & 13300 & 10953 & 70 \\
\hline $\mathrm{H}(28 \mathrm{D})$ & 6428 & 13271 & 10700 & 70 \\
\hline $\mathrm{H}(28 \mathrm{~A})$ & 618 & 2894 & 6439 & 64 \\
\hline $\mathrm{H}(28 \mathrm{~B})$ & 471 & 1940 & 6435 & 64 \\
\hline $\mathrm{H}(29 \mathrm{~A})$ & 795 & 1820 & 6991 & 54 \\
\hline $\mathrm{H}(29 \mathrm{~B})$ & 4829 & 12798 & 10633 & 64 \\
\hline $\mathrm{H}(30 \mathrm{~A})$ & 2066 & 1912 & 6700 & 80 \\
\hline $\mathrm{H}(30 \mathrm{~B})$ & 2173 & 2282 & 7059 & 80 \\
\hline $\mathrm{H}(30 \mathrm{C})$ & 2076 & 2862 & 6750 & 80 \\
\hline $\mathrm{H}(30 \mathrm{D})$ & 5168 & 14085 & 10458 & 129 \\
\hline $\mathrm{H}(30 \mathrm{E})$ & 4797 & 13558 & 10165 & 129 \\
\hline $\mathrm{H}(30 \mathrm{~F})$ & 5776 & 13721 & 10186 & 129 \\
\hline $\mathrm{H}(32 \mathrm{~A})$ & 6657 & 13119 & 10002 & 58 \\
\hline $\mathrm{H}(32 \mathrm{~B})$ & 7244 & 12359 & 10047 & 58 \\
\hline $\mathrm{H}(33 \mathrm{~A})$ & -372 & 1743 & 7394 & 35 \\
\hline $\mathrm{H}(33 \mathrm{~B})$ & 7584 & 13118 & 9584 & 68 \\
\hline $\mathrm{H}(34 \mathrm{~B})$ & 7182 & 12375 & 9115 & 77 \\
\hline $\mathrm{H}(34 \mathrm{~A})$ & -583 & 1075 & 7892 & 52 \\
\hline $\mathrm{H}(35 \mathrm{~B})$ & 5906 & 11588 & 9147 & 67 \\
\hline
\end{tabular}




\begin{tabular}{|c|c|c|c|c|}
\hline $\mathrm{H}(35 \mathrm{~A})$ & -14 & 1628 & 8372 & 47 \\
\hline $\mathrm{H}(36 \mathrm{~A})$ & 739 & 2785 & 8355 & 51 \\
\hline $\mathrm{H}(37 \mathrm{D})$ & 2963 & 11748 & 9272 & 107 \\
\hline $\mathrm{H}(37 \mathrm{E})$ & 3456 & 12152 & 9570 & 107 \\
\hline $\mathrm{H}(37 \mathrm{~F})$ & 3042 & 11288 & 9614 & 107 \\
\hline $\mathrm{H}(37 \mathrm{~A})$ & 3291 & 2922 & 8026 & 187 \\
\hline $\mathrm{H}(37 \mathrm{~B})$ & 2763 & 2814 & 7697 & 187 \\
\hline $\mathrm{H}(37 \mathrm{C})$ & 2379 & 2542 & 8041 & 187 \\
\hline $\mathrm{H}(38 \mathrm{~B})$ & 4426 & 11627 & 9189 & 54 \\
\hline $\mathrm{H}(38 \mathrm{~A})$ & 2679 & 4248 & 7859 & 105 \\
\hline $\mathrm{H}(39 \mathrm{C})$ & 3656 & 10056 & 9266 & 92 \\
\hline $\mathrm{H}(39 \mathrm{D})$ & 3970 & 10451 & 8929 & 92 \\
\hline $\mathrm{H}(39 \mathrm{~A})$ & 1828 & 3867 & 8479 & 215 \\
\hline $\mathrm{H}(39 \mathrm{~B})$ & 2692 & 4350 & 8455 & 215 \\
\hline $\mathrm{H}(40 \mathrm{C})$ & 5295 & 10248 & 9057 & 78 \\
\hline $\mathrm{H}(40 \mathrm{D})$ & 4820 & 9423 & 9127 & 78 \\
\hline $\mathrm{H}(40 \mathrm{~A})$ & 2118 & 5153 & 8141 & 246 \\
\hline $\mathrm{H}(40 \mathrm{~B})$ & 1736 & 5109 & 8504 & 246 \\
\hline $\mathrm{H}(41 \mathrm{~A})$ & 517 & 4292 & 8299 & 100 \\
\hline $\mathrm{H}(41 \mathrm{~B})$ & 4845 & 9608 & 9683 & 57 \\
\hline $\mathrm{H}(42 \mathrm{D})$ & 6279 & 9326 & 9533 & 119 \\
\hline $\mathrm{H}(42 \mathrm{E})$ & 6259 & 9907 & 9844 & 119 \\
\hline $\mathrm{H}(42 \mathrm{~F})$ & 6452 & 10262 & 9488 & 119 \\
\hline $\mathrm{H}(42 \mathrm{~A})$ & 251 & 5573 & 8187 & 325 \\
\hline $\mathrm{H}(42 \mathrm{~B})$ & -29 & 5035 & 7882 & 325 \\
\hline $\mathrm{H}(42 \mathrm{C})$ & 820 & 5532 & 7865 & 325 \\
\hline $\mathrm{H}(44 \mathrm{~A})$ & 2835 & 4624 & 6190 & 116 \\
\hline $\mathrm{H}(44 \mathrm{~B})$ & 4418 & 9869 & 10765 & 72 \\
\hline $\mathrm{H}(45 \mathrm{~A})$ & 1698 & 5087 & 5865 & 105 \\
\hline $\mathrm{H}(45 \mathrm{~B})$ & 4131 & 9759 & 11336 & 93 \\
\hline $\mathrm{H}(46 \mathrm{~A})$ & 428 & 5193 & 6114 & 87 \\
\hline $\mathrm{H}(46 \mathrm{~B})$ & 3997 & 10958 & 11650 & 125 \\
\hline $\mathrm{H}(47 \mathrm{~A})$ & 178 & 4898 & 6640 & 79 \\
\hline
\end{tabular}


1. Scriban, C.; Glueck, D. S. J. Am. Chem. Soc. 2006, 128, 2788-2789.

2. Scriban, C.; Glueck, D. S.; DiPasquale, A. G.; Rheingold, A. L. Organometallics 2006, 25, 54355448.

3. As described in more detail in the manuscript and in reference 2, four diastereomers of 4 were observed at low temperature; we were only able to study the NMR spectra of the major one in detail.

4. Neuhaus, D.; Williamson, M. P. The Nuclear Overhauser Effect in Structural and Conformational Analysis. VCH: New York, 1989; p. 358. 